\title{
Lacunary hyperbolic groups
}

\author{
ALEXANDER Yu OL'SHANSKII \\ DENIS V OSIN \\ MARK V SAPIR \\ APPENDIX BY MICHAEL KAPOVICH \\ APPENDIX BY BRUCE KLEINER
}

\begin{abstract}
We call a finitely generated group lacunary hyperbolic if one of its asymptotic cones is an $\mathbb{R}$-tree. We characterize lacunary hyperbolic groups as direct limits of Gromov hyperbolic groups satisfying certain restrictions on the hyperbolicity constants and injectivity radii. Using central extensions of lacunary hyperbolic groups, we solve a problem of Gromov by constructing a group whose asymptotic cone $\mathcal{C}$ has countable but nontrivial fundamental group (in fact $\mathcal{C}$ is homeomorphic to the direct product of a tree and a circle, so $\pi_{1}(\mathcal{C})=\mathbb{Z}$ ). We show that the class of lacunary hyperbolic groups contains non-virtually cyclic elementary amenable groups, groups with all proper subgroups cyclic (Tarski monsters) and torsion groups. We show that Tarski monsters and torsion groups can have so-called graded small cancellation presentations, in which case we prove that all their asymptotic cones are hyperbolic and locally isometric to trees. This allows us to solve two problems of Druţu and Sapir and a problem of Kleiner about groups with cut points in their asymptotic cones. We also construct a finitely generated group whose divergence function is not linear but is arbitrarily close to being linear. This answers a question of Behrstock.
\end{abstract}

20F65; 20F69

\section{Introduction}

\subsection{Asymptotic cones and lacunary hyperbolic groups}

Asymptotic cones were introduced by Gromov in [19]; a definition via ultrafilters was given by van den Dries and Wilkie [9]. An asymptotic cone of a metric space is, roughly speaking, what one sees when one looks at the space from infinitely far away. More precisely, any asymptotic cone of a metric space $(X$, dist) corresponds to an ultrafilter $\omega$, a sequence of observation points $e=\left(e_{n}\right)_{n \in \mathbb{N}}$ from $X$ and a sequence of scaling constants $d=\left(d_{n}\right)_{n \in \mathbb{N}}$ diverging to $\infty$. The cone $\operatorname{Con}^{\omega}(X ; e, d)$ corresponding to 
$\omega, e$ and $d$ is the ultralimit of the sequence of spaces with basepoints $\left(X, \mathrm{dist} / d_{n}, e_{n}\right)$ (see Section 2.3 for a precise definition).

In particular, if $X$ is the Cayley graph of a group $G$ with a word metric then the asymptotic cones of $X$ are called asymptotic cones of $G$. For every finitely generated group $G$, its asymptotic cones are complete geodesic homogeneous metric spaces. Since asymptotic cones of a group do not depend on the choice of observation points, we shall omit it from the notation.

The power of asymptotic cones stems from the fact that they capture both geometric and logical properties of the group, since a large subgroup $G_{e}^{\omega}$ of the ultrapower $G^{\omega}$ of the group $G$ acts transitively by isometries on the asymptotic cone $\operatorname{Con}^{\omega}(G ; d)$. Since a large part of the first order theory of $G$ is inherited by $G_{e}^{\omega}$, the isometry group $G_{e}^{\omega}$ of the asymptotic cone "looks" like $G$. One of the simple but fundamental applications of asymptotic cones is the following statement by Gromov [21]: if all asymptotic cones of a group are simply connected then the group has polynomial isoperimetric and linear isodiametric functions.

On the other hand, the asymptotic cone captures the coarse properties of the word metric in $G$. In particular, the asymptotic cones of two quasi-isometric groups are bi-Lipschitz equivalent. This makes asymptotic cones very useful tools in proving quasi-isometric rigidity of some classes of groups; see Kleiner and Leeb [30], Kapovich and Leeb [28], Kapovich, Kleiner and Leeb [27], Druţu [10; 11] and Druţu and Sapir [13].

Using asymptotic cones, one can characterize several important classes of groups. For example, groups of polynomial growth are precisely groups with all asymptotic cones locally compact; see Gromov [19], Druţu [11] and Point [48].

Another well-known result of Gromov is the following: a finitely generated group is hyperbolic if and only if all its asymptotic cones are $\mathbb{R}$-trees [20].

In fact, results of Gromov from [20] imply that a finitely presented group is hyperbolic if just one of the asymptotic cones is an $\mathbb{R}$-tree. This was discovered by Kapovich and Kleiner who give a detailed proof in the Appendix to this paper (see Theorem 8.1). On the other hand, there are nonhyperbolic finitely generated (but not finitely presented) groups with one asymptotic cone an $\mathbb{R}$-tree and another one not an $\mathbb{R}$-tree by Thomas and Velickovic [51]. We call a group lacunary hyperbolic if one of its asymptotic cones is an $\mathbb{R}$-tree. The term is originated in [22] where sparse sequences of relations satisfying certain small cancellation conditions as in [51] are called lacunary. Thus a finitely presented lacunary hyperbolic group is hyperbolic.

The following theorem characterizes lacunary hyperbolic groups as certain direct limits of hyperbolic groups. The proof is not too difficult modulo Theorem 8.1, but the result has never been formulated before. 
Let $\alpha: G \rightarrow G^{\prime}$ be a homomorphism, $G=\langle S\rangle$. The injectivity radius of $\alpha$ is the maximal radius of a ball in the Cayley graph $\Gamma(G, S)$ where $\alpha$ is injective.

Theorem 1.1 Let $G$ be a finitely generated group. Then the following conditions are equivalent.

(1) $G$ is lacunary hyperbolic.

(2) There exists a scaling sequence $d=\left(d_{n}\right)$ such that $\operatorname{Con}^{\omega}(G, d)$ is an $\mathbb{R}$-tree for any nonprincipal ultrafilter $\omega$.

(3) $G$ is the direct limit of a sequence of hyperbolic groups $G_{i}=\left\langle S_{i}\right\rangle\left(S_{i}\right.$ is finite) and epimorphisms

$$
G_{1} \stackrel{\alpha_{1}}{\longrightarrow} G_{2} \stackrel{\alpha_{2}}{\longrightarrow} \cdots
$$

where $\alpha_{i}\left(S_{i}\right)=S_{i+1}$, and the hyperbolicity constant of $G_{i}$ (relative to $S_{i}$ ) is "little $o$ " of the injectivity radius of $\alpha_{i}$.

Note that not every direct limit of hyperbolic groups is lacunary hyperbolic. For example, the free noncyclic Burnside group of any sufficiently large odd exponent and the wreath product $(\mathbb{Z} / n \mathbb{Z})$ wr $\mathbb{Z}$ are direct limits of hyperbolic groups (see Ivanov [25] and Osin [45], respectively) but are not lacunary hyperbolic by Druţu and Sapir [13].

Groups constructed by Thomas and Velickovic [51] and more general small cancellation groups given by relations whose lengths form lacunary sequences of numbers from Gromov [22, Section 1.7] are lacunary hyperbolic (see also Section 3.2 below).

In this paper, we prove that the class of lacunary hyperbolic groups is very large: non-virtually cyclic groups in that class can be elementary amenable, can have infinite centers, can have all proper subgroups cyclic (Tarski monsters) and can be torsion groups.

We also show (Theorem 4.17) that the class of lacunary hyperbolic groups contains all groups given by graded small cancellation presentations, a notion originated by the first author [41; 42]. Moreover, all asymptotic cones of groups given by graded small cancellation presentations are hyperbolic and locally isometric to trees (Theorem 4.17). Thus methods from $[41 ; 42]$ can be used to construct lacunary hyperbolic groups with unusual properties (see Section 4 and Section 6).

Theorem 1.1 implies that the torsion-free group $G$ with all proper subgroups cyclic from the paper [39] of the first author is lacunary hyperbolic. Indeed, $G$ is a direct limit of hyperbolic groups $G_{i}$ by [39, Lemma 9.13]. The same lemma shows that the injectivity radius of the homomorphism $G_{i-1} \rightarrow G_{i}$ can be chosen arbitrary large relative to the hyperbolicity constant of $G_{i-1}$. Similarly, the finitely generated infinite 
torsion group with all proper subgroups of prime orders constructed by the first author in [40] is lacunary hyperbolic as well.

Although the class of lacunary hyperbolic groups is very large, these groups share some common algebraic properties (see Sections 3.3 and 3.4). In particular in Theorem 3.18, Corollary 3.21 and Proposition 3.15, we show that

- an undistorted subgroup of a lacunary hyperbolic group is lacunary hyperbolic itself;

- a lacunary hyperbolic group cannot contain a copy of $\mathbb{Z}^{2}$, an infinite finitely generated subgroup of bounded torsion and exponential growth, a copy of the lamplighter group, etc.;

- every lacunary hyperbolic group is embedded into a relatively hyperbolic 2generated lacunary hyperbolic group as a peripheral subgroup;

- any group that is hyperbolic relative to a lacunary hyperbolic subgroup is lacunary hyperbolic itself.

Theorem 1.1 implies that lacunary hyperbolic groups satisfy the Strong Novikov Conjecture (that is the Baum-Connes assembly map with trivial coefficients is injective) since all direct limits of hyperbolic groups satisfy that conjecture (hyperbolic groups satisfy it by Skandalis, Tu and $\mathrm{Yu}$ [50], and direct limits respect the conjecture by Rosenberg [49, Proposition 2.4]).

It is also easy to see that the class of lacunary hyperbolic groups is closed under quasi-isometry: indeed, asymptotic cones of quasi-isometric groups are bi-Lipschitz equivalent if they correspond to the same ultrafilter and the same sequence of scaling constants. Hence if an asymptotic cone of one of these groups is an $\mathbb{R}$-tree, then the other group also has an asymptotic cone that is a $\mathbb{R}$-tree.

\subsection{Central extensions of lacunary hyperbolic groups and fundamental groups of asymptotic cones}

One of the interesting properties of hyperbolic groups was established by Neumann and Reeves [37]: every finitely generated central extension $H$ of a hyperbolic group $G$ is quasi-isometric to the direct product of $G$ and the center $Z(H)$. Using some general properties of asymptotic cones of group extensions (Theorem 5.2) we establish an asymptotic analog of this result for central extensions of lacunary hyperbolic groups.

Theorem 1.2 (Theorem 5.6) Let $N$ be a central subgroup of a finitely generated group $G$ endowed with the induced metric. Suppose that for some nonprincipal 
ultrafilter $\omega$ and some scaling sequence $d=\left(d_{n}\right)$, $\operatorname{Con}^{\omega}(G / N, d)$ is an $\mathbb{R}$-tree. Then $\operatorname{Con}^{\omega}(G, d)$ is bi-Lipschitz equivalent to $\operatorname{Con}^{\omega}(N, d) \times \operatorname{Con}^{\omega}(G / N, d)$ endowed with the product metric.

This theorem opens many opportunities to construct asymptotic cones of groups with unusual properties. Recall that one of the main problems about asymptotic cones of groups is the following question by Gromov.

Problem 1.3 (Gromov [21]) Is it true that the fundamental group of an asymptotic cone of a finitely generated group is either trivial or of cardinality continuum?

Here is what was known about Problem 1.3 before.

- As we have mentioned above, the triviality of the fundamental group of all asymptotic cones of a group $G$ implies that the group is finitely presented, its Dehn function is polynomial and its isodiametric function is linear. Thus fundamental groups of asymptotic cones carry important algorithmic information about the group.

- By Papasoglu [47] if the Dehn function of a finitely presented group is at most quadratic, then all asymptotic cones are simply connected. By Ol'shanskii and Sapir [43], one cannot replace in the previous statement "quadratic" by, say, $n^{2} \log n$.

- By Burillo [8], in many cases asymptotic cones of groups contain a $\pi_{1}$-embedded Hawaiian earring, and their fundamental groups are of order continuum (that is true, for example, for solvable Baumslag-Solitar groups and the Sol group).

- Non-simply connected asymptotic cones are non-locally compact by Druţu [11] and Point [48] but homogeneous, and the isometry groups act on them with uncountable point stabilizers. Hence every nontrivial loop in the asymptotic cone typically has uncountably many copies sharing a common point. This makes a positive answer to Problem 1.3 plausible.

- In all cases when the nontrivial fundamental groups of asymptotic cones of groups could be computed, these groups had cardinality continuum. Druţu and Sapir [13] proved that for every countable group $C$ there exists a finitely generated group $G$ and an asymptotic cone of $G$ whose fundamental group is the free product of continuously many copies of $C$.

Nevertheless, by carefully choosing a central extension of a lacunary hyperbolic group, we answer Problem 1.3 negatively. 
Theorem 1.4 (Theorem 5.12) There exists a finitely generated group $G$ and a scaling sequence $d=\left(d_{n}\right)$ such that for any ultrafilter $\omega, \operatorname{Con}^{\omega}(G, d)$ is bi-Lipschitz equivalent to the product of an $\mathbb{R}$-tree and $\mathbb{S}^{1}$. In particular, $\pi_{1}\left(\operatorname{Con}^{\omega}(G, d)\right)=\mathbb{Z}$.

\subsection{Cut points in asymptotic cones}

The examples of lacunary hyperbolic groups constructed in this paper solve several problems of Druţu and Sapir [13] and of Kleiner (see below).

Recall that one of the main applications of asymptotic cones of groups is the following: if a finitely generated group $H$ has infinitely many homomorphisms into a locally compact (say, finitely generated) group $G$ that are pairwise nonconjugate in $G$, then $H$ acts on an asymptotic cone of $G$ without a global fixed point. If the asymptotic cone is an $\mathbb{R}$-tree, this implies (using the theory of groups acting on $\mathbb{R}$-trees due to Rips, Sela, Bestvina and Feighn, Dunwoody and others) that $H$ splits into a graph of groups.

In [14], Druţu and Sapir showed that similar conclusions can be drawn if all asymptotic cones of $G$ are not trees but only have global cut points (ie points whose removal makes the cones disconnected). Such groups are called constricted. In that case the asymptotic cone is tree-graded in the sense of [13], and an action on a tree-graded space under some mild assumptions leads to an action on an $\mathbb{R}$-tree. In [14], this program has been carried out for relatively hyperbolic groups (all asymptotic cones of relatively hyperbolic groups have cut points by a result of Druţu and Sapir [13]). It is quite plausible that the program will work also for mapping class groups (where existence of cut points in asymptotic cones has been proved by Behrstock [5]), fundamental groups of graph manifolds (their asymptotic cones have cut points by a result of Kapovich, Kleiner and Leeb [27]), groups acting $k$-acylindrically on trees by Druţu, Mozes and Sapir [12] and other groups.

On the other hand many groups do not have cut points in any of their asymptotic cones. Such groups were called wide in [13]. Among them are non-virtually cyclic groups satisfying nontrivial laws [13], lattices in classical semisimple Lie groups of rank $>1$ [12], groups having infinite cyclic central subgroups [13], direct products of infinite groups and so on.

Metric spaces whose asymptotic cones do not have cut points (ie, wide spaces) are characterized internally in terms of divergence in [12]. A metric space is wide if and only if there are constants $C, \varepsilon>0$ such that for every three points $a, b, c$ there exist a path of length at most $C \operatorname{dist}(a, b)$ connecting $a$ with $b$ and avoiding a ball of radius $\varepsilon \operatorname{dist}(c,\{a, b\})$ about $c$. 
One can formulate this condition more precisely in terms of the divergence function of a metric space.

Definition 1.5 Let ( $X$, dist) be a 1 -ended geodesic metric space, $0<\delta<1, \lambda>0$. Let $a, b, c \in X, \min (\operatorname{dist}(c, a), \operatorname{dist}(c, b))=r . \operatorname{Define}_{\operatorname{Div}_{\lambda}}(a, b, c ; \delta)$ as the infimum of lengths of paths connecting $a, b$ and avoiding the ball Ball $(c, \delta r-\lambda)$ (a ball of nonpositive radius is defined to be empty). Now define the divergence function $\operatorname{Div}_{\lambda}(n ; \delta): \mathbb{R} \rightarrow \mathbb{R}$ of the space $X$ as the supremum of all numbers $\operatorname{Div}_{\lambda}(a, b, c ; \delta)$ where $\operatorname{dist}(a, b) \leq n$.

Clearly, the smaller $\delta$ and the bigger $\lambda$, the smaller the functions $\operatorname{Div}_{\lambda}(a, b, c ; \delta)$ and $\operatorname{Div}_{\lambda}(n ; \delta)$. For 1 -ended Cayley graphs, and any $\delta, \delta^{\prime}<\frac{1}{2}, \lambda, \lambda^{\prime}>2$, the functions $\operatorname{Div}_{\lambda}(n ; \delta)$ and $\operatorname{Div}_{\lambda^{\prime}}\left(n, \delta^{\prime}\right)$ are equivalent [12]. (Recall that two nondecreasing functions $f, g: \mathbb{N} \rightarrow \mathbb{N}$ are called equivalent if for some constant $C>1$, we have

$$
f(n / C-C)-C n-C \leq g(n)<f(C n+C)+C n+C
$$

for every $n$.)

Hence we can talk about the divergence function $\operatorname{Div}(n)$ of a 1-ended Cayley graph (setting $\delta=\frac{1}{3}, \lambda=2$ ).

It is proved in [12] that a 1-ended Cayley graph $X$ is wide if and only if $\operatorname{Div}(n)$ is bounded by a linear function; and asymptotic cones $\operatorname{Con}^{\omega}\left(X,\left(d_{n}\right)\right)$ do not have cut points for all $\omega$ if and only if for every $C>1$, the divergence function $\operatorname{Div}(n)$ is uniformly (in $n$ ) bounded by a linear function on the intervals $\left[d_{k} / C, C d_{k}\right]$.

The divergence function $\operatorname{Div}(n)$ is an interesting quasi-isometry invariant of a group. It is essentially proved in Alonso et al [2] that for every hyperbolic group $\operatorname{Div}(n)$ is at least exponential. On the other hand, for the mapping class groups (that also have cut points in all asymptotic cones; see Behrstock [5]), the divergence function is quadratic. The following question was asked by J Behrstock.

Problem 1.6 Does there exist a group with strictly subquadratic but not linear divergence function?

The answer to this question is given below.

If some asymptotic cones of a group $G$ do not have cut points, then the divergence function of $G$ can be estimated. 
Theorem 1.7 (Theorem 6.1) Let $G$ be a 1-ended finitely generated group. Suppose that for some sequence of scaling constants $d_{n}$ and every ultrafilter $\omega$, the asymptotic cone $\operatorname{Con}^{\omega}\left(G,\left(d_{n}\right)\right)$ does not have cut points. Let $f(n) \geq n$ be a nondecreasing function such that $d_{n} \leq f\left(d_{n-1}\right)$ for all sufficiently large $n$. Then the divergence function $\operatorname{Div}(n)$ of $G$ does not exceed $C f(n)$ for some constant $C$ (and all $n$ ).

An a priori stronger property than existence of cut points in asymptotic cones is the existence of the so-called Morse quasigeodesics in the Cayley graph of the group [12]. A quasigeodesic $q$ is called Morse if every $(L, C)$-quasigeodesic $p$ with endpoints on the image of $q$ stays $M$-close to $q$ where $M$ depends only on $L, C$. By the Morse lemma, every bi-infinite quasigeodesic of a hyperbolic space is Morse. It is proved in [12], that a quasigeodesic $q$ in a metric space $X$ is Morse if and only if in every asymptotic cone $\mathcal{C}$, and every point $m$ in the ultralimit $\bar{q}$ of $q$, the two halves of $\bar{q}$ (before $m$ and after $m$ ) are in two different connected components of $\mathcal{C} \backslash\{m\}$ (the implication " $\rightarrow$ " of this statement was proved in [5]).

Note that similar divergence properties of geodesics have been studied in the case of CAT(0) spaces with a cocompact group action by Ballmann [4] and Kapovich and Leeb [28]. In particular, linear (and even subquadratic) divergence for a locally compact Hadamard metric space (ie CAT( 0$)$, complete, geodesic, simply connected metric space) implies that every periodic bi-infinite geodesic in it bounds a flat halfplane (recall that a geodesic is called periodic if it is stable under an isometry of the space that acts nontrivially on the geodesic). Ballmann proved [4, Theorem 3.5] that if a CAT(0) space $X$ has at least 3 points on the boundary and contains a bi-infinite periodic geodesic that does not bound a flat half-plane, then any sufficiently large (say, cocompact) group of isometries of $X$ contains a free non-Abelian subgroup.

This leads to the following two problems from [13]. Recall that a finitely generated group is constricted if all its asymptotic cones have cut points.

Problem 1.8 [13, Problem 1.17] Is every nonconstricted group wide? In other words, if one of the asymptotic cones of a group has no cut points, does every asymptotic cone of the group have no cut points?

Problem 1.9 [13, Problem 1.19] Does every non-virtually cyclic finitely generated constricted group contain free non-Abelian subgroups? Is there a constricted group with all proper subgroups cyclic?

Bruce Kleiner asked the following stronger questions: 
Problem 1.10 (Kleiner) Can a finitely generated group $G$ without free noncyclic subgroups contain a bi-infinite Morse quasigeodesic that is periodic? Is there a nonwide amenable non-virtually cyclic group?

We show that the answers to these questions are affirmative.

The second part of Problem 1.10 is answered by the following result (because nontrivial trees have cut points).

Theorem 1.11 (Theorem 3.25, Lemma 3.23) There exists a finitely generated lacunary hyperbolic non-virtually cyclic elementary amenable group $G$. The group $G$ satisfies the following additional properties:

- $G$ is 2-generated.

- $G$ is (locally nilpotent $p$-group)-by-(infinite cyclic).

- $G$ is residually (finite $p$-group), in particular it is residually nilpotent.

Note that since $G$ is not hyperbolic (being amenable and non-virtually cyclic), not all of its asymptotic cones are $\mathbb{R}$-trees. Hence we obtain the first example of an amenable group with two nonhomeomorphic asymptotic cones.

The following result gives a solution of the first half of that problem and of Problem 1.9 .

Recall that a geodesic metric space $X$ is called tree-graded with respect to a collection of connected proper subsets $\mathcal{P}$ [13] if any two distinct subsets from $\mathcal{P}$ intersect by at most one point, and every nontrivial simple geodesic triangle of $X$ is contained in one of the sets from $\mathcal{P}$. In particular, if subsets from $\mathcal{P}$ are circles (with the natural length metric) of diameters bounded both from above and from below, we call $X$ a circle-tree. It is easy to see that every circle-tree is a hyperbolic space.

It is proved in [13] that every (nonsingleton) space that is tree-graded with respect to proper subspaces has cut points. Conversely, every geodesic space with cut points is tree-graded with respect to the collection of maximal connected subsets without (their own) cut points [13].

Theorem 1.12 (Theorem 4.26, Remark 4.27) There exist two lacunary hyperbolic non-virtually cyclic groups $Q_{1}$ and $Q_{2}$ such that all asymptotic cones of $Q_{i}$ are circle-trees and

(1) $Q_{1}$ is a torsion group; 
(2) every proper subgroup of $Q_{2}$ is infinite cyclic and every infinite periodic path in the Cayley graph of $Q_{2}$ is a Morse quasigeodesic.

Note that circle-trees are locally isometric to trees and are hyperbolic, so all the asymptotic cones of all groups from Theorem 1.12 are locally isometric hyperbolic spaces. But since some of the asymptotic cones of $Q_{i}$ are trees and some are not, not all of the cones are homeomorphic.

The group $Q_{1}$ is a torsion group but the exponents of elements in $Q_{1}$ are not bounded. By the cited result from [13] about groups satisfying a law, asymptotic cones of infinite torsion groups of bounded exponent do not have cut points.

The following theorems give two solutions of Problem 1.8. The first theorem uses central extensions of lacunary hyperbolic groups again.

Although any finitely generated group with infinite central cyclic subgroup is wide [13], the next theorem shows that one can construct lacunary hyperbolic groups with infinite (torsion) centers. Such a group has an asymptotic cone with cut points (a tree) and an asymptotic cone without cut points.

In fact the information we get is much more precise.

Theorem 1.13 (Theorem 5.11) For every $m \geq 2$, there exists a finitely generated central extension $G$ of a lacunary hyperbolic group such that for any ultrafilter $\omega$ and any scaling sequence $d=\left(d_{n}\right)$, exactly one of the following possibilities occurs and both of them can be realized for suitable $\omega$ and $d$.

(a) $\operatorname{Con}^{\omega}(G, d)$ is an $m$-fold cover of a circle-tree, the fibers of that cover are cut sets, and every finite cut set of $\operatorname{Con}^{\omega}(G, d)$ contains one of the fibers.

(b) $\operatorname{Con}^{\omega}(G, d)$ is an $\mathbb{R}$-tree.

In particular, in both cases $\operatorname{Con}^{\omega}(G, d)$ is locally isometric to an $\mathbb{R}$-tree.

Theorem 1.14 (Theorem 6.3) There exists a finitely generated torsion lacunary hyperbolic group $G$ such that one of the asymptotic cones of $G$ does not have cut points.

The construction from the proof of Theorem 6.3 allows us to answer Problem 1.6. Indeed, by carefully choosing exponents of elements of the group $G$ we can control the scaling constants in the asymptotic cones without cut points. Using Theorem 1.7, we prove (Corollary 6.4) that for every function $f(n)$ with $f(n) / n$ nondecreasing, $\lim f(n) / n=\infty$, there exists a finitely generated torsion group $G$ whose divergence function $\operatorname{Div}(n)$ is 
- not linear but bounded by a linear function on an infinite subset of $\mathbb{N}$;

- bounded from above by $C f(n)$ for some constant $C$ and all $n$.

In addition, one can arrange that the orders of elements $x \in G$ grow with the length $|x|$ as $O(g(|x|)$ for any prescribed in advance nondecreasing unbounded function $g(n)$. (Recall that groups with bounded torsion are wide [13].)

\subsection{Plan of the paper}

Section 2 (Preliminaries) contains the main properties of tree-graded spaces (this makes this paper as independent of [13] as possible) and the definition and main properties of asymptotic cones. It also contain some useful properties of hyperbolic groups.

Section 3 starts with the definition and basic properties of lacunary hyperbolic groups. In particular, we show (Lemma 3.1) that a group is lacunary hyperbolic provided it has a hyperbolic asymptotic cone, or an asymptotic cone that is locally isometric to an $\mathbb{R}$-tree. Then we prove the characterization of lacunary hyperbolic groups (Theorem 3.3).

The easiest examples of lacunary hyperbolic nonhyperbolic groups are groups given by certain infinite small cancellation presentations. Proposition 3.12 characterizes such presentations.

In Section 3.3 we present several observations connecting relative hyperbolicity and lacunar hyperbolicity. In particular Proposition 3.15 shows that a group that is hyperbolic relative to a lacunary hyperbolic subgroup is lacunary hyperbolic itself. This implies that every lacunary hyperbolic group embeds into a 2-generated lacunary hyperbolic group (quasi-isometrically, malnormally and even as a peripheral subgroup). This result cannot be generalized to several subgroups: we show (Example 3.16) that even a free product of two lacunary hyperbolic groups can be non-lacunary hyperbolic.

In Section 3.4, we provide several general properties of subgroups of lacunary hyperbolic groups already mentioned above in Section 1.1.

In Section 3.5, we construct elementary amenable lacunary hyperbolic groups and prove Theorem 1.11.

Section 4 is devoted to several small cancellation conditions and their applications. We start by introducing a small cancellation condition $C(\varepsilon, \mu, \rho)$ for presentations over any group $H$ (ie presentations of factor groups of $H$ instead of just factor-groups of the free group as in the classical case). We show (Lemma 4.6) that if the group $H$ is hyperbolic and the cancellation parameters are appropriately chosen, then the 
factor-group satisfies an analog of the Greendlinger lemma and is hyperbolic again with a nice control on the hyperbolicity constant.

This allows us to use induction and introduce direct limits of groups $G_{1} \rightarrow G_{2} \rightarrow \cdots$ where each $G_{i+1}$ is given by a presentation over $G_{i}$ which satisfies an appropriate $C(\varepsilon, \mu, \rho)$-condition so that Lemma 4.6 holds. The union of presentations of all $G_{i}$ gives us a presentation of the limit group $G$. We say that such a presentation satisfies a graded small cancellation condition. We prove (Corollary 4.15) that many infinitely presented groups with classical small cancellation conditions have graded small cancellation presentations.

Theorem 4.17 gives an important property of graded small cancellation presentations: every asymptotic cone of a group given by a graded small cancellation presentation is a circle-tree or an $\mathbb{R}$-tree. Moreover, given the parameters of the cone, one can tell which of these options holds and what are the sizes of the circles in the circle-tree.

In Section 4.4, we apply results of the first author [42] and show that there are nonvirtually cyclic groups with graded small cancellation presentations that have all proper subgroups infinite cyclic or all proper subgroups finite (Theorem 4.26, Remark 4.27).

In Section 4.5, we notice that existence of cut points in all asymptotic cones follows from the nontriviality of the Floyd boundary of a group. The converse statement does not hold as follows from Theorem 4.26.

In Section 5, we first establish some very general results about asymptotic cones of group extensions. In particular (Theorem 5.2), if

$$
1 \rightarrow N \rightarrow G \rightarrow H \rightarrow 1
$$

is an exact sequence then there exists a continuous map from a cone of $G$ to a cone of $H$ (corresponding to the same parameters) with fibers homeomorphic to the cone of $N$ (considered as a subspace of $G$ ). In the case of central extensions, the situation is much nicer, and in particular when the cone of $H$ is an $\mathbb{R}$-tree, the fibration becomes trivial and the cone of $G$ becomes bi-Lipschitz equivalent to the direct product of the $\mathbb{R}$-tree and the cone of $N$ (Theorem 5.6). As applications of these general results, we give proofs of Theorems 1.4 and 1.13 .

Section 6 is devoted to torsion groups and the proofs of Theorem 1.14 and Corollary 6.4 solving the slow divergence problem.

Section 7 contains some open problems.

The Appendix written by M Kapovich and B Kleiner contains the proof of Theorem 8.1 (that a finitely presented lacunary hyperbolic group is hyperbolic) and other useful results about asymptotic cones of finitely presented groups. 
Acknowledgements The authors are grateful to Cornelia Druţu and Bruce Kleiner for very fruitful conversations. We are also grateful to Michael Kapovich and Bruce Kleiner for adding their unpublished results as an Appendix to our paper.

The first and third authors were supported in part by the NSF grants DMS 0245600 and DMS 0455881. The second author was supported in part by the NSF grant DMS 0605093. In addition, the research of the first and second authors was supported in part by the Russian Fund for Basic Research grant 05-01-00895, and the research of the third author was supported by a BSF (USA-Israeli) grant.

\section{Preliminaries}

\subsection{Cayley graphs and van Kampen diagrams}

Given a word $W$ in an alphabet $S$, we denote by $|W|$ its length. We also write $W \equiv V$ to express the letter-for-letter equality of words $W$ and $V$.

Let $G$ be a group generated by a set $S$. Recall that the Cayley graph $\Gamma(G, S)$ of a group $G$ with respect to the set of generators $S$ is an oriented labeled 1-complex with the vertex set $V(\Gamma(G, S))=G$ and the edge set $E(\Gamma(G, S))=G \times S^{ \pm 1}$. An edge $e=(g, a)$ goes from the vertex $g$ to the vertex $g a$ and has label $\operatorname{Lab}(e) \equiv a$. As usual, we denote the initial and the terminal vertices of the edge $e$ by $e_{-}$and $e_{+}$ respectively. Given a combinatorial path $p=e_{1} \cdots e_{k}$ in the Cayley graph $\Gamma(G, S)$, where $e_{1}, \ldots, e_{k} \in E(\Gamma(G, S))$, we denote by $\operatorname{Lab}(p)$ its label. By definition, $\operatorname{Lab}(p) \equiv \operatorname{Lab}\left(e_{1}\right) \cdots \operatorname{Lab}\left(e_{k}\right)$. We also denote by $p_{-}=\left(e_{1}\right)_{-}$and $p_{+}=\left(e_{k}\right)_{+}$ the initial and terminal vertices of $p$ respectively. The length $|p|$ of $p$ is the number of edges in $p$.

The (word) length $|g|$ of an element $g \in G$ with respect to the generating set $S$ is defined to be the length of a shortest word in $S$ representing $g$ in $G$. The formula $\operatorname{dist}(f, g)=\left|f^{-1} g\right|$ defines a metric on $G$. We also denote by dist the natural extension of the metric to $\Gamma(G, S)$.

Recall that a van Kampen diagram $\Delta$ over a presentation

$$
G=\langle S \mid \mathcal{R}\rangle
$$

is a finite oriented connected planar 2-complex endowed with a labeling function Lab: $E(\Delta) \rightarrow S^{ \pm 1}$, where $E(\Delta)$ denotes the set of oriented edges of $\Delta$, such that $\operatorname{Lab}\left(e^{-1}\right) \equiv(\operatorname{Lab}(e))^{-1}$. Given a cell $\Pi$ of $\Delta$, we denote by $\partial \Pi$ the boundary of $\Pi$; similarly, $\partial \Delta$ denotes the boundary of $\Delta$. The labels of $\partial \Pi$ and $\partial \Delta$ are defined up to cyclic permutations. An additional requirement is that the label of any cell $\Pi$ of $\Delta$ is 
equal to (a cyclic permutation of) a word $R^{ \pm 1}$, where $R \in \mathcal{R}$. Labels and lengths of paths are defined as in the case of Cayley graphs.

The van Kampen Lemma states that a word $W$ over an alphabet $S$ represents the identity in the group given by (1) if and only if there exists a connected simply connected planar diagram $\Delta$ over (1) such that $\operatorname{Lab}(\partial \Delta) \equiv W[33$, Chapter 5, Theorem 1.1].

\subsection{Tree-graded spaces}

Here we collect all the necessary definitions and basic properties of tree-graded spaces from [13] needed in this paper.

Definition 2.1 Let $\mathbb{F}$ be a complete geodesic metric space and let $\mathcal{P}$ be a collection of closed geodesic nonempty subsets (called pieces). Suppose that the following two properties are satisfied:

$\left(T_{1}\right)$ Every two different pieces have at most one common point.

$\left(T_{2}\right)$ Every nontrivial simple geodesic triangle (a simple loop composed of three geodesics) in $\mathbb{F}$ is contained in one piece.

Then we say that the space $\mathbb{F}$ is tree-graded with respect to $\mathcal{P}$.

For technical reasons it is convenient to allow $\mathcal{P}$ to be empty. Clearly $\mathbb{F}$ is tree-graded with respect to the empty collections of pieces only if $\mathbb{F}$ is a tree.

By [13, Proposition 2.17], property $\left(T_{2}\right)$ in this definition can be replaced by each of the following two properties.

$\left(T_{2}^{\prime}\right)$ For every topological arc $c:[0, d] \rightarrow \mathbb{F}$, where $c(0) \neq c(d)$, and any $t \in[0, d]$, let $c[t-a, t+b]$ be a maximal subarc of $c$ containing $c(t)$ and contained in one piece. Then every other topological arc with the same endpoints as $c$ must contain the points $c(t-a)$ and $c(t+b)$.

$\left(T_{2}^{\prime \prime}\right)$ Every simple loop in $\mathbb{F}$ is contained in one piece.

In order to avoid problems with pieces that are singletons, we shall always assume that pieces in a tree-graded space cannot contain each other.

Let us define a partial order relation on the set of tree-graded structures of a space. If $\mathcal{P}$ and $\mathcal{P}^{\prime}$ are collections of subsets of $X$, and a space $X$ is tree-graded with respect to both $\mathcal{P}$ and $\mathcal{P}^{\prime}$, we write $\mathcal{P} \prec \mathcal{P}^{\prime}$ if for every set $M \in \mathcal{P}$ there exists $M^{\prime} \in \mathcal{P}^{\prime}$ such that $M \subset M^{\prime}$. The relation $\prec$ is a partial order because by our convention pieces of $\mathcal{P}$ (resp. $\mathcal{P}^{\prime}$ ) cannot contain each other. 


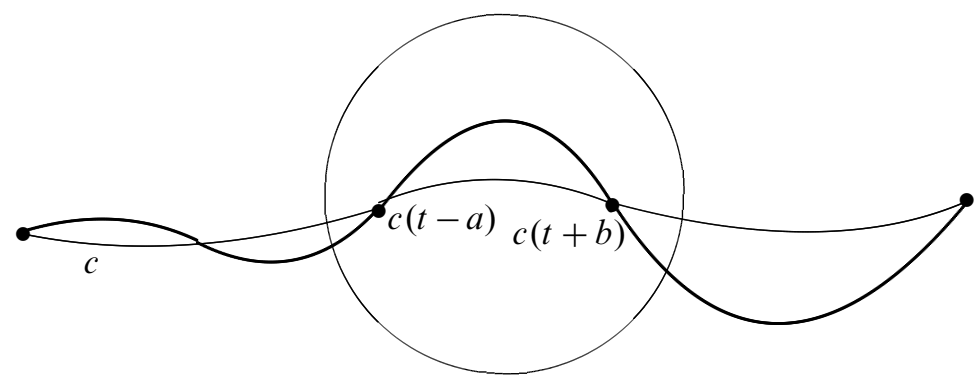

Figure 1: Property $\left(T_{2}^{\prime}\right)$

Lemma 2.2 [13, Lemma 2.31] Let $X$ be a complete geodesic metric space containing at least two points and let $\mathcal{C}$ be a nonempty set of global cut points in $X$.

(a) There exists the largest in the sense of $\prec$ collection $\mathcal{P}$ of subsets of $X$ such that - $X$ is tree-graded with respect to $\mathcal{P}$;

- any piece in $\mathcal{P}$ is either a singleton or a set with no global cut-point from $\mathcal{C}$. Moreover the intersection of any two distinct pieces from $\mathcal{P}$ is either empty or a point from $\mathcal{C}$.

(b) Let $X$ be a homogeneous space with a cut-point. Then every point in $X$ is a cut-point, so let $\mathcal{C}=X$. Let $\mathcal{P}$ be the set of pieces defined in (a). Then for every $M \in \mathcal{P}$ every $x \in M$ is the projection of a point $y \in X \backslash M$ onto $M$ (ie the closest to $y$ point in $M$ ).

Lemma 2.3 [13, Lemma 2.15] Let $\mathbb{F}$ be a tree-graded metric space. Let $A$ be a path connected subset of $\mathbb{F}$ without cut points. Then $A$ is contained in a piece. In particular every simple loop in $\mathbb{F}$ is contained in a piece of $\mathbb{F}$.

Lemma 2.4 [13, Lemma 2.28] Let $g=g_{1} g_{2} \ldots g_{2 m}$ be a curve in a tree-graded space $\mathbb{F}$ which is a composition of geodesics. Suppose that all geodesics $g_{2 k}$ with $k \in\{1, \ldots, m-1\}$ are nontrivial and for every $k \in\{1, \ldots, m\}$ the geodesic $g_{2 k}$ is contained in a piece $M_{k}$ while for every $k \in\{0,1, \ldots, m-1\}$ the geodesic $g_{2 k+1}$ intersects $M_{k}$ and $M_{k+1}$ only in its respective endpoints. In addition assume that if $g_{2 k+1}$ is empty then $M_{k} \neq M_{k+1}$. Then $g$ is a geodesic.

Lemma 2.5 [13, Corollary 2.10] (1) Every simple path in $\mathbb{F}$ joining two points in a piece is contained in the piece.

(2) Every nonempty intersection between a simple path in $\mathbb{F}$ and a piece is a subpath. 
Lemma 2.6 [13, Corollary 2.11] Let $A$ be a connected subset (possibly a point) in a tree-graded space $\mathbb{F}$ which intersects a piece $M$ in at most one point.

(1) The subset $A$ projects onto $M$ in a unique point $x$.

(2) Every path joining a point in $A$ with a point in $M$ contains $x$.

\subsection{Asymptotic cones}

Let us recall the definition of asymptotic cones. A nonprincipal ultrafilter $\omega$ is a finitely additive measure defined on all subsets $S$ of $\mathbb{N}$, such that $\omega(S) \in\{0,1\}$, $\omega(\mathbb{N})=1$, and $\omega(S)=0$ if $S$ is a finite subset. For a bounded sequence of numbers $x_{n}, n \in \mathbb{N}$, the $\operatorname{limit} \lim ^{\omega} x_{n}$ with respect to $\omega$ is the unique real number $a$ such that $\omega\left(\left\{i \in \mathbb{N}:\left|x_{i}-a\right|<\epsilon\right\}\right)=1$ for every $\epsilon>0$. Similarly, $\lim ^{\omega} x_{n}=\infty$ if $\omega\left(\left\{i \in \mathbb{N}: x_{i}>M\right\}\right)=1$ for every $M>0$.

Given two infinite sequences of real numbers $\left(a_{n}\right)$ and $\left(b_{n}\right)$ we write $a_{n}=o_{\omega}\left(b_{n}\right)$ if $\lim ^{\omega} a_{n} / b_{n}=0$. Similarly $a_{n}=\Theta_{\omega}\left(b_{n}\right)$ (respectively $\left.a_{n}=O_{\omega}\left(b_{n}\right)\right)$ means that $0<\lim ^{\omega}\left(a_{n} / b_{n}\right)<\infty$ (respectively $\left.\lim ^{\omega}\left(a_{n} / b_{n}\right)<\infty\right)$.

Let $\left(X_{n}\right.$, dist $\left._{n}\right), n \in \mathbb{N}$, be a metric space. Fix an arbitrary sequence $e=\left(e_{n}\right)$ of points $e_{n} \in X_{n}$. Consider the set $\mathcal{F}$ of sequences $g=\left(g_{n}\right), g_{n} \in X_{n}$, such that $\operatorname{dist}_{n}\left(g_{n}, e_{n}\right) \leq c$ for some constant $c=c(g)$. Two sequences $\left(f_{n}\right)$ and $\left(g_{n}\right)$ of this set $\mathcal{F}$ are said to be equivalent if $\lim ^{\omega} \operatorname{dist}_{n}\left(f_{n}, g_{n}\right)=0$. The equivalence class of $\left(g_{n}\right)$ is denoted by $\left(g_{n}\right)^{\omega}$. The $\omega$-limit $\lim ^{\omega}\left(X_{n}\right)_{e}$ is the quotient space of equivalence classes where the distance between $\left(f_{n}\right)^{\omega}$ and $\left(g_{n}\right)^{\omega}$ is defined as $\lim ^{\omega} \operatorname{dist}\left(f_{n}, g_{n}\right)$.

An asymptotic cone $\operatorname{Con}^{\omega}(X, e, d)$ of a metric space $\left(X\right.$, dist) where $e=\left(e_{n}\right), e_{n} \in X$, and $d=\left(d_{n}\right)$ is an unbounded nondecreasing scaling sequence of positive real numbers, is the $\omega$-limit of spaces $X_{n}=\left(X\right.$, dist $\left./ d_{n}\right)$. The asymptotic cone is a complete space; it is a geodesic metric space if $X$ is a geodesic metric space [21;11]. Note that $\operatorname{Con}^{\omega}(X, e, d)$ does not depend on the choice of $e$ if $X$ is homogeneous (say, if $X$ is a finitely generated group with a word metric), so in that case, we shall omit $e$ in the notation of an asymptotic cone.

If $\left(Y_{n}\right)$ is a sequence of subsets of $X$ endowed with the induced metric, we define $\lim ^{\omega}\left(Y_{n}\right)_{e}$ to be the subset of $\operatorname{Con}^{\omega}(X, e, d)$ consisting of $x \in \operatorname{Con}^{\omega}(X, e, d)$ that can be represented by sequences $\left(x_{n}\right)$, where $x_{n} \in Y_{n}$.

An asymptotic cone of a finitely generated group $G$ with a word metric is the asymptotic cone of its Cayley graph (considered as the discrete space of vertices with the word metric). Asymptotic cones corresponding to two different finite generating sets of $G$ (and the same ultrafilters and scaling constants) are bi-Lipschitz equivalent. The 
asymptotic cone $\operatorname{Con}^{\omega}(G, d)$ of a group $G$ is a homogeneous geodesic metric space with transitive group of isometries $G_{e}^{\omega}(d)$ consisting of sequences $\left(g_{n}\right), g_{n} \in G$ such that $\left|g_{n}\right| \leq C d_{n}$ for some constant $C$ depending on the sequence (here $\left|g_{n}\right|$ is the word length of $\left.g_{n}\right)$. The action is by multiplication on the left: $\left(g_{n}\right) \circ\left(h_{n}\right)^{\omega}=\left(g_{n} h_{n}\right)^{\omega}$.

Recall that a geodesic $p$ in $\operatorname{Con}^{\omega}(X ; e, d)$ is called a limit geodesic if $p=\lim ^{\omega} p_{n}$, where for every $n \in \mathbb{N}, p_{n}$ is a geodesic in $X$. The lemma below was proved in [10, Corollary 4.18].

Lemma 2.7 Assume that in an asymptotic cone $\operatorname{Con}^{\omega}(X ; e, d)$, a collection of closed subsets $\mathcal{P}$ satisfies $\left(T_{1}\right)$ and every nontrivial simple triangle in $\operatorname{Con}^{\omega}(X ; e, d)$ whose sides are limit geodesics is contained in a subset from $\mathcal{P}$. Then $\mathcal{P}$ satisfies $\left(T_{2}\right)$, ie, $\operatorname{Con}^{\omega}(X ; e, d)$ is tree-graded with respect to $\mathcal{P}$.

\subsection{Hyperbolic groups}

Recall that a geodesic space $X$ is $\delta$-hyperbolic (or simply hyperbolic, for brevity) if for any geodesic triangle $\Delta$ in $X$, each side of $\Delta$ is contained in the closed $\delta-$ neighborhood of the union of the other two sides. This $\delta$ is called the hyperbolicity constant of $X$. A group $H$ is $\delta$-hyperbolic (or simply hyperbolic) if it is generated by a finite set $S$ and its Cayley graph $\Gamma(H, S)$ endowed with the combinatorial metric is a hyperbolic metric space.

Recall that a path $p$ is called $(\lambda, c)$-quasigeodesic for some $\lambda \in(0,1], c \geq 0$ if for any subpath $q$ of $p$, we have

$$
\operatorname{dist}\left(q_{-}, q_{+}\right) \geq \lambda|q|-c .
$$

The property of hyperbolic spaces stated below is well-known although it is usually formulated in a slightly different manner (see, for example, Ghys and de la Harpe [18]).

Lemma 2.8 For any $\lambda \in(0,1], c>0$ there exists $\theta(\lambda, c)$ such that any two $(\lambda, c)-$ quasigeodesic paths $p, q$ in a $\delta$-hyperbolic metric space such that $p_{-}=q_{-}$and $p_{+}=q_{+}$belong to the closed $\theta(\lambda, c)$-neighborhoods of each other.

The next property can easily be derived from the definition of a hyperbolic space by cutting the $n$-gon into triangles.

Lemma 2.9 For any $n \geq 3$, any side of a geodesic $n$-gon in a $\delta$-hyperbolic space belongs to the closed $(n-2) \delta$-neighborhood of the other $(n-1)$ sides. 


\section{Lacunary hyperbolic groups: characterization and exam- ples}

\subsection{A characterization of lacunary hyperbolic groups}

We say that a metric space $X$ is lacunary hyperbolic if one of the asymptotic cones of $X$ is an $\mathbb{R}$-tree. In particular, every hyperbolic metric space is lacunary hyperbolic. A group $G$ is lacunary hyperbolic if it is finitely generated and the corresponding Cayley graph is lacunary hyperbolic. Clearly this notion is independent of the choice of the finite generating set. We also say that a metric space $X$ is almost homogeneous if there is a homogeneous subspace $Y \subseteq X$ such that $\operatorname{dist}_{\mathrm{Hau}}(X, Y)<\infty$, where dist ${ }_{\mathrm{Hau}}$ is the Hausdorff distance between $X$ and $Y$. That is, there exists $\varepsilon>0$ such that for every $x \in X$, we have $\operatorname{dist}(x, Y)<\varepsilon$. Given a group $G$, any Cayley graph of $G$ endowed with the combinatorial metric is almost homogeneous. Note also that if $X$ is almost homogeneous, then every asymptotic cone of $X$ is homogeneous.

Lemma 3.1 Let $X$ be a metric space. Then the following properties are equivalent.

(1) Some asymptotic cone of $X$ is an $\mathbb{R}$-tree.

(2) Some asymptotic cone of $X$ is lacunary hyperbolic.

If, in addition, $X$ is almost homogeneous, these properties are equivalent to:

(3) Some asymptotic cone of $X$ is locally isometric to an $\mathbb{R}$-tree.

Proof It suffices to show that (2) $\Rightarrow(1)$ and (3) $\Rightarrow(1)$. Recall that the set $\mathcal{C}(X)$ of asymptotic cones of $X$ is closed under taking ultralimits [13, Corollary 3.24]. Further let dist denote the standard metric on $\operatorname{Con}^{\omega}(X ; e, d)$. It is straightforward to check that for every $k>0,\left(\operatorname{Con}^{\omega}(X ; e, d), \frac{1}{k}\right.$ dist $)$ is isometric to the asymptotic cone $\operatorname{Con}\left(X, e,\left(k d_{n}\right)\right)$. In particular, if $Y \in \mathcal{C}(X)$, then every asymptotic cone of $Y$ belongs to $C(X)$. This yields $(2) \Rightarrow(1)$.

Further if $\operatorname{Con}^{\omega}(X ; e, d)$ is homogeneous and locally isometric to an $\mathbb{R}$-tree, there is $c>0$ such that $\operatorname{Con}^{\omega}(X ; e, d)$ contains no simple nontrivial loops of length at most $c$. We consider the sequence of cones $C_{k}=\operatorname{Con}\left(X, e,\left(k d_{n}\right)\right)$ for $k \rightarrow 0$. Clearly $C_{k}$ has no simple nontrivial loops of length at most $c / k$. Then for any nonprincipal ultrafilter $\omega, \lim ^{\omega}\left(C_{k}\right)_{e}$ is an $\mathbb{R}$-tree.

By Theorem 8.1 of Kapovich and Kleiner, if a finitely presented group $G$ is lacunary hyperbolic, then, in fact, it is hyperbolic. Combining this with Lemma 3.1, we obtain the following. 
Proposition 3.2 The following conditions are equivalent for any finitely presented group $G$.

(1) Some asymptotic cone of $G$ is lacunary hyperbolic.

(2) Some asymptotic cone of $G$ is locally isometric to an $\mathbb{R}$-tree.

(3) All asymptotic cones of $G$ are $\mathbb{R}$-trees, ie, $G$ is hyperbolic.

In contrast, in Sections 4-5 we construct a nonhyperbolic finitely generated group all of whose asymptotic cones are quasi-isometric and locally isometric to an $\mathbb{R}$-tree.

The next theorem describes the structure of lacunary hyperbolic groups. Given a group homomorphism $\alpha: G \rightarrow H$ and a generating set $S$ of $G$, we denote by $r_{S}(\alpha)$ the injectivity radius of $\alpha$ with respect to $S$, ie, the radius of the largest ball $B$ in $G$ such that $\alpha$ is injective on $B$.

Theorem 3.3 Let $G$ be a finitely generated group. Then the following conditions are equivalent.

(1) $G$ is lacunary hyperbolic.

(2) There exists a scaling sequence $d=\left(d_{n}\right)$ such that $\operatorname{Con}^{\omega}(G, d)$ is an $\mathbb{R}$-tree for any nonprincipal ultrafilter $\omega$.

(3) $G$ is the direct limit of a sequence of finitely generated groups and epimorphisms

$$
G_{1} \stackrel{\alpha_{1}}{\longrightarrow} G_{2} \stackrel{\alpha_{2}}{\longrightarrow} \cdots
$$

such that $G_{i}$ is generated by a finite set $S_{i}, \alpha_{i}\left(S_{i}\right)=S_{i+1}$, and each $G_{i}$ is $\delta_{i}$-hyperbolic, where $\delta_{i}=o\left(r_{S_{i}}\left(\alpha_{i}\right)\right)$.

Proof We are going to show that $(2) \Rightarrow(1) \Rightarrow(3) \Rightarrow(2)$. The first implication is trivial. Let us prove the second one. Suppose that $\operatorname{Con}^{\omega}(G, d)$ is an $\mathbb{R}$-tree for some $\omega$ and nondecreasing $d=\left(d_{n}\right)$. For every $n$, we denote by $H_{n}$ the group given by the presentation $\left\langle S \mid \mathcal{R}_{n}\right\rangle$, where $\mathcal{R}_{n}$ consists of labels of all cycles in the ball of radius $d_{n}$ around the identity in $\Gamma(G, S)$. Note that $G$ is a quotient of $H_{n}$ and the canonical map $H_{n} \rightarrow G$ is injective on the ball of radius $d_{n}$. It follows that the natural epimorphisms $H_{n} \rightarrow H_{m}$ are also injective on the balls of radius $d_{n}$ for arbitrary $m \geq n$.

Observe that by Kapovich and Kleiner's Theorem 8.3 and Lemma 8.2 from the Appendix, there are constants $C_{1}, C_{2}, C_{3}$ with the following property. Let $H$ be a group having a finite presentation $\langle S \mid \mathcal{R}\rangle, D=\max _{R \in \mathcal{R}}|R|$. Assume that for some $\delta$ and $d \geq \max \left\{C_{1} \delta, D\right\}$, every ball of radius $C_{2} d$ in $\Gamma(H, S)$ is $\delta$-hyperbolic. Then $\Gamma(H, S)$ is $C_{3} d$-hyperbolic. 
Since $\operatorname{Con}^{\omega}(G, d)$ is an $\mathbb{R}$-tree, balls of radius $d_{n}$ in $\Gamma(G, S)$ (and hence in $\Gamma\left(H_{n}, S\right)$ ) are $o_{\omega}\left(d_{n}\right)$-hyperbolic. Recall that any $\delta$-hyperbolic graph endowed with the combinatorial metric becomes 1 -connected after gluing 2-cells along all combinatorial loops of length at most $16 \delta$ (see, for example, Bridson and Haefliger [7, Chapter III.H, Lemma 2.6]). Hence $H_{n}$ admits a finite presentation with generating set $S$ and relations of lengths $o_{\omega}\left(d_{n}\right)$. In particular, for every positive integer $k$, there exists $n(k)$ such that the sequence $(n(k))$ is strictly increasing and the following conditions hold.

(H1) $\quad H_{n(k)}$ admits a finite presentation with generating set $S$ and relations of lengths at most $d_{n(k)} /\left(C_{2} k\right)$.

(H2) Every ball of radius $d_{n(k)} / k$ in the $\Gamma\left(H_{n(k)}, S\right)$ is $d_{n(k)} /\left(C_{1} C_{2} k\right)$-hyperbolic.

These conditions allow us to apply the above observation to $H=H_{n(k)}, d=D=$ $d_{n(k)} /\left(C_{2} k\right)$, and $\delta=d_{n(k)} /\left(C_{1} C_{2} k\right)$. Thus $H_{n(k)}$ is $C_{3} d_{n(k)} /\left(C_{2} k\right)$-hyperbolic. Now setting $G_{k}=H_{n(k)}$ we obtain a sequence of groups and homomorphisms

$$
G_{1} \stackrel{\alpha_{1}}{\longrightarrow} G_{2} \stackrel{\alpha_{2}}{\longrightarrow} \cdots
$$

where $G_{k}$ is $\delta_{k}$-hyperbolic for $\delta_{k}=C_{3} d_{n(k)} /\left(C_{2} k\right)=o\left(d_{n(k)}\right)$ and $\alpha_{k}$ is injective on the ball of radius $d_{n(k)}$ as desired.

To prove $(3) \Rightarrow(2)$ we fix any sequence $d=\left(d_{n}\right)$ such that

$$
\delta_{n}=o\left(d_{n}\right), \quad d_{n}=o\left(r_{S}\left(\alpha_{n}\right)\right) .
$$

Let $\omega$ be an arbitrary nonprincipal ultrafilter. According to Lemma 2.7 applied to the collection $\mathcal{P}$ of all one-element subsets of $\operatorname{Con}^{\omega}(G, d)$, to show that $\operatorname{Con}^{\omega}(G, d)$ is a tree it suffices to prove that it contains no simple nontrivial limit geodesic triangles.

Suppose that $p q s$ is a nontrivial simple triangle in $\operatorname{Con}^{\omega}(G, d)$ whose sides are limit geodesics. Clearly $p q s=\lim ^{\omega} H_{n}$, where $H_{n}=p_{n} a_{n} q_{n} b_{n} s_{n} c_{n}$ is a geodesic hexagon in $\Gamma(G, S)$ such that $p=\lim ^{\omega} p_{n}, q=\lim ^{\omega} q_{n}, s=\lim ^{\omega} s_{n}$,

$$
\left|a_{n}\right|=o_{\omega}\left(d_{n}\right), \quad\left|b_{n}\right|=o_{\omega}\left(d_{n}\right), \quad\left|c_{n}\right|=o_{\omega}\left(d_{n}\right),
$$

and perimeter of $H_{n}$ satisfies $\left|H_{n}\right|=O_{\omega}\left(d_{n}\right)$. By (3) we have $\left|H_{n}\right|=o\left(r_{S}\left(\alpha_{n}\right)\right)$, hence the label of $H_{n}$ represents 1 in $G_{n} \omega$-almost surely. Thus $H_{n}$ may be considered as a configuration in the Cayley graph of $G_{n} \omega$-almost surely.

Let $p$ be a nontrivial side of $p q s$. Lemma 2.9 implies that $p_{n}$ belongs to the closed $4 \delta_{n}$-neighborhood of the other sides $\omega$-almost surely. Combining this with (4) and (3), we obtain that the $\left(O_{\omega}\left(\delta_{n}\right)+o_{\omega}\left(d_{n}\right)\right)$-neighborhood of $q_{n} \cup r_{n}$ contains $p_{n}$. Since $O_{\omega}\left(\delta_{n}\right)=o_{\omega}\left(d_{n}\right), p$ belongs to the union of $q$ and $r$. 
Remark 3.4 It is easy to see from the proof of Theorem 3.3 that (3) can be replaced with the following (a priori stronger) condition, which will be useful for some applications.

(4) $G$ is the direct limit of a sequence of finitely generated groups and epimorphisms

$$
G_{1} \stackrel{\alpha_{1}}{\longrightarrow} G_{2} \stackrel{\alpha_{2}}{\longrightarrow} \cdots
$$

such that $G_{i}$ is generated by a finite set $S_{i}, \alpha_{i}\left(S_{i}\right)=S_{i+1}$, each $G_{i}$ is $\delta_{i}$-hyperbolic, $\alpha_{i}$ is injective on the ball of radius $r_{i}$ of the group $G_{i}=\left\langle S_{i}\right\rangle$, where $\delta_{i}=o\left(r_{i}\right)$ and

(4a) the sequence of the numbers $r_{i}$ is nondecreasing;

(4b) the group $G_{i}$ has a presentation $\left\langle S_{i} \mid \mathcal{P}_{i}\right\rangle$, where $\max _{P \in \mathcal{P}_{i}}|P|=o\left(r_{i}\right)$.

Indeed it is easy to see that the proof of the implication $(1) \Rightarrow(3)$ ensures (d1) for $r_{i}=d_{n(i)}$ and (d2) follows from Condition (H1).

Remark 3.5 The third condition from the theorem implies that every lacunary hyperbolic group (as well as any other limit of hyperbolic groups) embeds into an ultraproduct of hyperbolic groups. Indeed let $\phi: G_{1} \rightarrow \prod^{\omega} G_{i}$ be the homomorphism defined by the rule $\phi(g)=\left(\alpha_{1}(g), \alpha_{2} \circ \alpha_{1}(g), \ldots\right)$. If $\omega$ is nonprincipal, it is straightforward to see that $\operatorname{Ker}(\phi)=\bigcup_{i=1}^{\infty} \operatorname{Ker}\left(\alpha_{i} \circ \cdots \circ \alpha_{1}\right)$ and hence $\phi\left(G_{1}\right) \cong G$. In particular, if a universal sentence holds in the first order group language holds in all hyperbolic groups, then it holds in all lacunary hyperbolic groups.

This observations is similar to Mal'cev's Local Theorems [34]. It provides us with a uniform way of proving (nontrivial) universal theorems for lacunary hyperbolic groups. As an example, the reader may verify that the sentence

$$
\forall x \forall y\left(x^{-1} y^{2} x=y^{3} \Rightarrow\left[y, x^{-1} y x\right]=1\right)
$$

is a theorem in the class of all hyperbolic group and hence in the class of all lacunary hyperbolic groups, but it is not a theorem in the class of all groups.

\subsection{Lacunary hyperbolic groups and the classical small cancellation con- dition}

We begin with examples of lacunary hyperbolic groups constructed by means of the classical small cancellation theory. Recall the small cancellation condition $C^{\prime}(\mu)$. Given a set $\mathcal{R}$ of words in a certain alphabet, one says that $\mathcal{R}$ is symmetrized if for any $R \in \mathcal{R}$, all cyclic shifts of $R^{ \pm 1}$ are contained in $\mathcal{R}$. 


\section{Definition 3.6 Let}

$$
G=\langle S \mid \mathcal{R}\rangle
$$

be a group presentation, where $\mathcal{R}$ is a symmetrized set of reduced words in a finite alphabet $S$. A common initial subword of any two distinct words in $\mathcal{R}$ is called a piece. We say that $\mathcal{R}$ satisfies the $C^{\prime}(\mu)$ if any piece contained (as a subword) in a word $R \in \mathcal{R}$ has length smaller than $\mu|R|$.

The main property of groups with $C^{\prime}(\mu)$-presentations is given by the Greendlinger Lemma below.

Lemma 3.7 [33, Theorem V.4.4.] Let $\mathcal{R}$ be a symmetrized set of words in a finite alphabet $S$ satisfying a $C^{\prime}(\mu)$ condition with $\mu \leq 1 / 6, \mathcal{P}=\langle S \mid \mathcal{R}\rangle$. Assume that a reduced van Kampen diagram $\Delta$ over $\mathcal{P}$ with cyclically reduced boundary path $q$ has at least one cell. Then $q$ and the boundary path of some cell $\Pi$ in $\Delta$ have a common subpath $t$ with $|t|>(1-3 \mu)|\partial \Pi|$.

Given a van Kampen diagram $\Delta$ over (5), we denote by $\mathcal{A}(\Delta)$ the sum of the perimeters of all cells in $\Delta$. The next lemma easily follows from the Greendlinger Lemma by induction on the number of cells in the diagram.

Lemma 3.8 Suppose that a group presentation (5) satisfies the $C^{\prime}(\mu)$-condition for some $\mu \leq 1 / 6$. Then for any reduced diagram $\Delta$ over (5), we have

(a) $|\partial \Delta|>(1-3 \mu)|\partial \Pi|>|\partial \Pi| / 2$ for any cell $\Pi$ in $\Delta$;

(b) $|\partial \Delta|>(1-6 \mu) \mathcal{A}(\Delta)$.

The lemma below was actually proved in [2] although it was not stated explicitly there. The explicit statement is due to Ollivier [38].

Lemma 3.9 Suppose that there exists $C>0$ such that for every minimal van Kampen diagram $\Delta$ over (5), we have $|\partial \Delta| \geq C \mathcal{A}(\Delta)$. Then provided $G$ is finitely presented, it is $\delta$-hyperbolic, where $\delta \leq 12 \max _{R \in \mathcal{R}}|R| / C^{2}$.

Corollary 3.10 If a finite group presentation (5) satisfies the $C^{\prime}(\mu)$-condition for some $\mu<1 / 6$. Then $G$ is $\delta$-hyperbolic, where $\delta \leq 12 \max _{R \in \mathcal{R}}|R| /(1-6 \mu)^{2}$.

Definition 3.11 We say that a subset $L \subset \mathbb{N}$ is sparse, if for any $\lambda>0$, there exists a segment $I=[a, b] \subset[1,+\infty)$ such that $I \cap L=\varnothing$ and $a / b<\lambda$. 
Given a (not necessary finite) presentation (5), we denote by $L(\mathcal{R})$ the set $\{|R| \mid R \in \mathcal{R}\}$. The following result provides us with a rich source of examples of lacunary hyperbolic groups.

Proposition 3.12 Let (5) be a group presentation with finite alphabet $S$, satisfying the $C^{\prime}(\mu)$ small cancellation condition for some $\mu<1 / 6$. Then the group $G$ is lacunary hyperbolic if and only if the set $L(\mathcal{R})$ is sparse.

Proof Suppose that $L(\mathcal{R})$ is sparse. Then for every $n \in \mathbb{N}$, there exists a segment $I_{n}=\left[a_{n}, b_{n}\right] \subset \mathbb{R}$ such that

$$
I_{n} \cap L(\mathcal{R})=\varnothing,
$$

$b_{n} / a_{n}>n$, and $a_{n+1}>b_{n}$. We set $\mathcal{R}_{n}=\left\{R \in \mathcal{R}|| R \mid \leq a_{n}\right\}$ and $G_{n}=\left\langle S \mid \mathcal{R}_{n}\right\rangle$. Then $G$ is the limit of the sequence of the groups $G_{n}$ and the obvious homomorphisms $\alpha_{n}: G_{n} \rightarrow G_{n+1}$. By Corollary 3.10, $G_{n}$ is $\delta_{n}$-hyperbolic, where $\delta_{n}=O\left(a_{n}\right)=o\left(b_{n}\right)$. On the other hand, by Lemma 3.8 (a) and (6) we have $r_{S}\left(\alpha_{n}\right) \geq b_{n} / 2$. Hence $G$ is lacunary hyperbolic by Theorem 3.3.

Now assume that $G$ is lacunary hyperbolic. Let $G=\lim G_{i}$ as in Remark 3.4. Then, in the notation of Remark 3.4, we have $r_{i} \geq \lambda\left(2 M_{i}+1\right)$ for given $\lambda>0$ and all sufficiently large $i$, where $M_{i}=\max _{P \in \mathcal{P}_{i}}|P|$. To prove that $L(\mathcal{R})$ is sparse, it suffices to show that there is no $R \in \mathcal{R}$ with $r_{i} \geq|R| \geq 2 M_{i}+1$. By Condition (d1) in Remark 3.4, the natural homomorphism $G_{i} \rightarrow G$ is also injective on balls of radius $r_{i}$. Hence $R=1$ in $G_{i}$. However, by Lemma 3.8 (a), all words from $\mathcal{P}_{i}$ represent 1 in the group $G_{i}^{\prime}=\left\langle S \mid \mathcal{T}_{i}\right\rangle$, where $\mathcal{T}_{i}=\left\{R \in \mathcal{R}|| R \mid \leq 2 M_{i}\right\}$. Hence $R=1$ in $G_{i}^{\prime}$. As $R \notin \mathcal{T}_{i}$, this contradicts Lemma 3.7 and the $C^{\prime}(\mu)$-condition.

\subsection{Relative and lacunar hyperbolicity}

Recall a definition of relatively hyperbolic groups. There are at least six equivalent definitions (the first one is due to Gromov [21]). We use the definition whose equivalence to the other definitions is proved in [13].

Definition 3.13 [13, Theorem 8.5] Let $G$ be a finitely generated group, $H_{1}, \ldots, H_{n}$ subgroups of $G$. Then $G$ is called (strongly) hyperbolic relative to peripheral subgroups $H_{1}, \ldots, H_{n}$ if every asymptotic cone $\operatorname{Con}^{\omega}(G, d)$ of $G$ is tree-graded with respect to the collection of nonempty ultralimits of sequences of left cosets $\lim ^{\omega}\left(g_{j} H_{i}\right), g_{j} \in G$, where different sequences of cosets $\left(g_{j} H_{i}\right),\left(g_{j}^{\prime} H_{i}\right)$ define the same piece if they coincide $\omega$-almost surely. 
Remark 3.14 Recall that in a finitely generated relatively hyperbolic group $G$, every peripheral subgroup is finitely generated and quasi-isometrically embedded in $G$ [46]. This implies that each ultralimit $\lim ^{\omega}\left(g_{j} H_{i}\right)$ is either empty or bi-Lipschitz equivalent to the asymptotic cone $\operatorname{Con}^{\omega}\left(H_{i}, d\right)$ with respect to a finite generating set of $H_{i}$ (see Druţu and Sapir [13] for details).

The first two claims of the following proposition provides us with a way of constructing new lacunary hyperbolic groups from given ones. The third claim is related to the following problem. It is well-known that if a group $G$ is hyperbolic relative to a finitely presented subgroup $H$, then $G$ is finitely presented itself. However it is still unknown (see [46, Problem 5.1]) whether finite presentability of $G$ implies finite presentability of $H$ (although $H$ is finitely generated whenever $G$ is [46]). An ultimate negation of this implication would be the following statement:

Any finitely generated recursively presented group $H$ embeds into a finitely presented relatively hyperbolic group $G$ as a peripheral subgroup.

Proposition 3.12 and Proposition 3.15 (c) imply that this statement does not hold.

Proposition 3.15 $\quad$ (a) If a finitely generated group $G$ is hyperbolic relative to a lacunary hyperbolic subgroup $H$, then $G$ is itself lacunary hyperbolic.

(b) Every lacunary hyperbolic group $H$ embeds into a 2-generated lacunary hyperbolic group $G$. Moreover one can assume that $G$ is hyperbolic relative to $H$.

(c) If $G$ is hyperbolic relative to a lacunary hyperbolic subgroup $H$ and $H$ is not finitely presented, then $G$ is not finitely presented.

Proof (a) Suppose that $G$ is hyperbolic relative to $H$ and $\operatorname{Con}^{\omega}(H, d)$ is an $\mathbb{R}$-tree. By Definition 3.13 and Remark 3.14, $\operatorname{Con}^{\omega}(G, d)$ is tree-graded relative to a collection of $\mathbb{R}$-trees. In particular, $\operatorname{Con}^{\omega}(G, d)$ has no nontrivial simple loops, ie, it is an $\mathbb{R}$-tree.

(b) By [3, Theorem 1.1] applied to the free group of rank 2, there exists a 2-generated group $G$ such that $H$ embeds in $G$ and $G$ is hyperbolic relative to $H$. It remains to use part (a).

(c) The group $G$ is lacunary hyperbolic by (a). If $G$ was finitely presented, it would be hyperbolic by Kapovich and Kleiner's Theorem 8.1 from the Appendix. Since peripheral subgroups are quasi-isometrically embedded into relatively hyperbolic groups [46] and quasi-isometrically embedded subgroups of hyperbolic groups are hyperbolic [2], $\mathrm{H}$ is hyperbolic. Hence $H$ is finitely presented that contradicts our assumption. 
Observe that the first assertion of Proposition 3.15 cannot be generalized to the case of several peripheral subgroups. Moreover, we have the following.

Example 3.16 The free product $H_{1} * H_{2}$ of lacunary hyperbolic groups is not necessarily lacunary hyperbolic. Indeed it is not hard to construct a set of words $\mathcal{R}=\left\{R_{i}, i \in \mathbb{N}\right\}$ in a finite alphabet $S$ such that $\left|R_{i}\right|=i$ and $\mathcal{R}$ satisfies the $C^{\prime}(1 / 7)$ condition. It is also easy to find two subsets $N_{1}, N_{2} \subset \mathbb{N}$ such that both $N_{1}, N_{2}$ are sparse and $N_{1} \cup N_{2}=\mathbb{N}$. Set

$$
H_{1}=\left\langle S \mid R_{i}=1, i \in N_{1}\right\rangle, \quad H_{2}=\left\langle S \mid R_{i}=1, i \in N_{2}\right\rangle .
$$

Then by Proposition $3.12 H_{1}, H_{2}$ are lacunary hyperbolic while $H_{1} * H_{2}$ is not.

\subsection{Subgroups of lacunary hyperbolic groups}

The next theorem shows that subgroups of lacunary hyperbolic groups share common properties with subgroups of hyperbolic groups.

Definition 3.17 Let $H$ be a subgroup of a finitely generated group $G=\langle S\rangle$. Then the growth function of $H$ (relative to $S$ ) is the function

$$
f_{H, G}(n)=\#\left(\operatorname{Ball}_{G}(n) \cap H\right)
$$

where $\operatorname{Ball}_{G}(n)$ is the ball of radius $n$ around 1 in the group $G$ (in the word metric related to $S$. We say that $H$ has exponential growth in $G$ if its growth function $f_{H, G}(n)$ is bounded from below by an exponent $d^{n}$ for some $d>1$.

Theorem 3.18 Let $G$ be a lacunary hyperbolic group.

(a) Every finitely presented subgroup of $G$ is a subgroup of a hyperbolic group.

(b) Every undistorted (ie quasi-isometrically embedded) subgroup of $G$ is lacunary hyperbolic.

(c) Let $H$ be a (not necessarily finitely generated) bounded torsion subgroup of $G$. Then the growth of $H$ in $G$ is not exponential.

Proof (a) Indeed, every finitely presented subgroup of a lacunary hyperbolic group $G$ that is a direct limit of hyperbolic groups $G_{i}$ as in Theorem 3.3 is isomorphic to a subgroup of one of the $G_{i}$ 's.

Remark 3.19 It is worth noting that for the same reason, every finitely presented subgroup of the free Burnside group $B$ of any sufficiently large odd exponent is cyclic because $B$ is a direct limit of hyperbolic groups [25], periodic subgroups of hyperbolic groups are finite [20], and finite subgroups of $B$ are cyclic [1]. 
(b) If $H$ is a finitely generated undistorted subgroup of a lacunary hyperbolic group $G$ then every asymptotic cone $\operatorname{Con}^{\omega}\left(H,\left(d_{n}\right)\right)$ of $H$ is bi-Lipschitz homeomorphic to a subspace of the corresponding asymptotic cone $\operatorname{Con}^{\omega}\left(G,\left(d_{n}\right)\right)$. Since a connected subspace of an $\mathbb{R}$-tree is an $\mathbb{R}$-tree, every undistorted subgroup of a lacunary hyperbolic group is lacunary hyperbolic itself.

(c) Let $G=\langle S\rangle$ be a direct limit of hyperbolic groups $G_{i}=\left\langle S_{i}\right\rangle$ and homomorphisms $\alpha_{i}$ as in Remark 3.4, $S=S^{-1}, S_{i}=S_{i}^{-1}$ Note that the volume of the ball Ball $G_{i}(r)$ of radius $r$ in $G_{i}$ is at most $a^{r}$ for $a=\# S+1$.

Suppose $H \leq G$ has exponential growth in $G$ and bounded torsion: $h^{n}=1$ for all $h \in H$. Denote by $H_{i}$ the preimage of $H$ in $G_{i}$. Since $H$ has exponential growth in $G$, there is $d>1$ such that the number of elements from $H_{i}$ of length at most $t$ in the generators $S_{i}$ is at least $d^{t}$ for every integer $t$. We denote this subset by $B_{i}(t)$.

Suppose for some $i$ and $t_{0}$, all the elements of $B_{i}\left(2 t_{0}\right)$ have finite orders. Then all elements of both $B_{i}\left(t_{0}\right)$ and $B_{i}\left(t_{0}\right) B_{i}\left(t_{0}\right)$ have finite orders. It is proved in [26, Lemma 17] that under these assumptions the subset $B_{i}\left(t_{0}\right)$ must be conjugate in the hyperbolic group $G_{i}$ to a subset of $\mathrm{Ball}_{G_{i}}\left(205 \delta_{i}\right)$. Therefore $d^{t_{0}} \leq \operatorname{card} B_{i}\left(t_{0}\right) \leq$ $a^{205 \delta_{i}}$, and so $t_{0} \leq C \delta_{i}$, for $C=205 \log _{d} a$. Hence there is a constant $D \geq 2 C$ such that in every intersection $H_{i} \cap \mathrm{Ball}_{G_{i}}\left(D \delta_{i}\right)$, there exists an element $g_{i}$ of infinite order.

Since $H$ has exponent $\leq n$, the epimorphism $G_{i} \rightarrow G$ is not injective on the ball of radius $\left|g_{i}^{n}\right| \leq n D \delta_{i}$ for every $i$. Hence in the notation of Remark 3.4, we have $r_{i}<n D \delta_{i}$. Therefore $\delta_{i} / r_{i}>1 /(n D)$ for all $i$ and (c) is proved by contradiction.

Remark 3.20 Note that both the growth condition and the bounded torsion condition are essential in Theorem 3.18(c). Indeed, Theorem 5.11 gives examples of lacunary hyperbolic groups with infinite (central) subgroups of any given finite exponent $\geq 2$; Theorem 3.25 below provides an example of lacunary hyperbolic group with infinite locally finite (torsion) normal subgroup, and lacunary hyperbolic groups from Theorem 4.26 (1) and Theorem 6.3 are torsion themselves.

Corollary 3.21 (a) A lacunary hyperbolic group $G$ cannot contain copies of $\mathbb{Z}^{2}$ or Baumslag-Solitar groups.

(b) The free Burnside group $B(m, n)$ with sufficiently large exponent $n$ and $m \geq 2$ cannot be a subgroup of a lacunary hyperbolic group.

(c) The lamplighter group $(\mathbb{Z} / 2 \mathbb{Z}) \mathrm{wr} \mathbb{Z}$ cannot be a subgroup of a lacunary hyperbolic group. 
Proof (a) It follows from Theorem 3.18 (a). Indeed $\mathbb{Z}^{2}$ and Baumslag-Solitar groups are finitely presented and are not subgroups of hyperbolic groups

(b) Indeed, $B(m, n)$ has exponential growth for any $m \geq 2, n \gg 1$ [25]. Hence it has exponential growth in any group $G$ containing $B(m, n)$ and we can use Theorem 3.18 (c).

(c) Consider any short exact sequence

$$
1 \rightarrow N \rightarrow G \rightarrow Q \rightarrow 1
$$

where $G=\langle S\rangle, Q=\langle S N\rangle$. Let $f_{N, G}$ be the growth function of $N$ in $G, f_{G}$ and $f_{Q}$ be the growth functions of $G$ and $Q$ relative to the generating sets $S$ and $S N$ respectively. Then the following inequality obviously holds:

$$
f_{G}(n) \leq f_{N, G}(2 n) f_{Q}(n)
$$

for every $n \geq 1$. Applying this inequality to the short exact sequence

$$
1 \rightarrow N \rightarrow(\mathbb{Z} / 2 \mathbb{Z}) \mathrm{wr} \mathbb{Z} \rightarrow \mathbb{Z} \rightarrow 1
$$

where $N=(\mathbb{Z} / 2 \mathbb{Z})^{\mathbb{Z}}$ is the base of the wreath product, we deduce that $N$ has exponential growth in the lamplighter group, and, consequently, in any finitely generated group containing the lamplighter group. Since $N$ has exponent 2 , it cannot be a subgroup of a lacunary hyperbolic group by Theorem 3.18 (c).

Remark 3.22 It is clear that the same argument shows that a lacunary hyperbolic group has no finitely generated subgroup $H$ of exponential growth which is an extension of a bounded torsion subgroup by a nilpotent group.

\subsection{Lacunary hyperbolic amenable groups}

Recall that the class of elementary amenable groups is defined to be the smallest class containing all finite and Abelian groups and closed under taking directed unions, extensions, quotients, and subgroups. In this section, we will construct an elementary amenable group some of whose asymptotic cones are $\mathbb{R}$-trees.

Pick a prime number $p$ and a nondecreasing sequence $\mathbf{c}$ of positive integers $c_{1} \leq c_{2} \leq \cdots$. Consider the group $A=A(p, \mathbf{c})$ generated by $a_{i}, i \in \mathbb{Z}$ subject to the following relations:

$$
\begin{gathered}
a_{i}^{p}=1, \quad i \in \mathbb{Z}, \\
{\left[\ldots\left[a_{i_{0}}, a_{i_{1}}\right], \ldots, a_{i_{c_{n}}}\right]=1}
\end{gathered}
$$


for every $n$ and all commutators with $\max _{j, k}\left|i_{j}-i_{k}\right| \leq n$. The group $A=A(p, \mathbf{c})$ is locally nilpotent since arbitrary $a_{j}, a_{j+1}, \ldots, a_{j+n}$ generate a nilpotent subgroup of nilpotency class at most $c_{n}$. Since the locally nilpotent group $A$ is generated by elements of order $p$, it is a $p$-group [23]. Notice that for $l \geq 0$, there is a retraction $\pi_{l}$ of $A$ onto the finite subgroup $A(l)$ generated by $a_{0}, \ldots, a_{l}\left(\pi_{l}\left(a_{j}\right)=1\right.$ if $\left.j \neq 0, \ldots l\right)$.

The group $A$ admits the automorphism $a_{i} \rightarrow a_{i+1}(i \in \mathbb{Z})$. Denote by $G=G(p, \mathbf{c})$ the extension of $A$ by this automorphism, ie, $G$ is generated by the normal subgroup $A$ and an element $t$ of infinite order such that $t a_{i} t^{-1}=a_{i+1}$ for every integer $i$.

Lemma 3.23 The group $G=G(p, \mathbf{c})$ satisfies the following properties:

(a) $G$ is 2-generated.

(b) $G$ is (locally nilpotent $p$-group)-by-(infinite cyclic), and so it is elementary amenable.

(c) $G$ admits an epimorphism onto the wreath product $(\mathbb{Z} / p \mathbb{Z})$ wr $\mathbb{Z}$ and so $G$ is not virtually cyclic.

(d) $G$ is residually (finite $p$-group).

Proof (a) It is clear that $G$ is generated by $a_{0}$ and $t$.

(b) and (c) follow form the construction.

(d) Fix $m=p^{s}$ for some $s>0$ and consider a "circular version" $B$ of the group $A$, namely the (finite) group $B$ generated by $m$ elements $b_{[i]}$, where $[i]$ is a residue class modulo $m$, with defining relations $b_{[i]}^{p}=1$ and $\left[\ldots\left[b_{\left[i_{0}\right]}, b_{\left[i_{1}\right]}\right], \ldots, b_{\left[i_{c_{n}}\right]}\right]=1$ for every $n$ and all commutators with $\max _{j, k}\left|\left[i_{j}-i_{k}\right]\right| \leq n$, where $|[i]|$ is the smallest nonnegative integer $s$ such that either $i-s$ or $i+s$ is 0 modulo $m$. The group $B$ is a finite $p$-group.

Notice that if $0 \leq l \leq m / 2$, then $B$ has a retraction on the subgroup $B(l)$ generated by $b_{[0]}, \ldots, b_{[l]}$ which are subject to all the defining relations of $B$ involving these generators. Since $l \leq m / 2$, we have $\left|\left[i_{j}-i_{k}\right]\right|=\left|i_{j}-i_{k}\right|$ for $i_{j}, i_{k} \in\{0, \ldots, l$, and so the group $B(l)$ is naturally isomorphic with $A(l)$.

We have an epimorphism $\alpha_{m}: A \rightarrow B=B_{m}$ such that $\alpha_{m}\left(a_{i}\right)=b_{[i]}$ for all $a_{i}$ 's, and, by the previous observation, this homomorphism maps the subgroup $A(l) \leq A$ isomorphically onto the subgroup $B(l) \leq B$ provided $l \leq m / 2$.

Let $H_{m}$ be the extension of $B=B_{m}$ by the automorphism of order $m: t_{m}^{-1} b_{[i]} t_{m}=$ $b_{[i+1]}, t_{m}^{m}=1$. We have $\left|H_{m}\right|=m\left|B_{m}\right|$, so $H_{m}$ is a $p$-group. Then the epimorphism $\alpha_{m}$ extends to the epimorphism $\beta_{m}$ of $G$ onto $H_{m}$ such that $\beta_{m}(t)=t_{m}$. The 
intersection of kernels of arbitrary infinite family of homomorphisms $\beta_{m}$ contains no nontrivial elements from $A(l), l=0,1,2, \ldots$, and so it is trivial. Hence $G$ is a residually (finite $p$-group).

Lemma 3.24 The groups $G(p, \mathbf{c})$ are limits of hyperbolic (in fact virtually free) groups satisfying all assumptions of Theorem 3.3, provided the sequence $\mathbf{c}$ grows fast enough.

Proof We chose a sequence $\mathbf{c}=\left(c_{1}, c_{2}, \ldots\right)$ by induction.

Let $C_{0}=*_{i=-\infty}^{\infty}\left\langle a_{i} \mid a_{i}^{p}=1\right\rangle, c_{1}=1$. Suppose that the integers $c_{1} \leq \cdots \leq c_{n}$ are already chosen. For every $l \leq n$, we denote by $U_{l}$ the normal subgroup of $C_{0}$ generated (as a normal subgroup) by all commutators of the form

$$
\left[\ldots\left[a_{i_{0}}, a_{i_{1}}\right], \ldots, a_{i_{c_{l}}}\right]
$$

where $\max _{j, k}\left|i_{j}-i_{k}\right| \leq l$. Then we set $V_{n}=\prod_{l=1}^{n} U_{l}$ and $C_{n}=C_{0} / V_{n}$.

The map $a_{i} \rightarrow a_{i+1}, i=0,1, \ldots$, extends to an automorphism of $C_{n}$. Let

$$
G_{n}=\left\langle C_{n}, t \mid a_{i}^{t}=a_{i+1}, i \in \mathbb{Z}\right\rangle
$$

be the corresponding extension by the automorphism. Clearly $G_{n}$ is generated by $\left\{a_{0}, t\right\}$, and the set of defining relations of $G_{n-1}$ is a subset of the set of defining relations of $G_{n}$ (and the set of defining relations of $G$ ). Thus the identity map on this set induces epimorphisms

$$
G_{1} \rightarrow G_{2} \rightarrow \cdots \rightarrow G_{n-1} \rightarrow G_{n} \rightarrow G .
$$

Observe that $G_{n}$ splits as an HNN-extension of its subgroup generated by $a_{0}, \ldots, a_{n}$. This subgroup is nilpotent of class at most $c_{n}$ and generated by elements of order $p$, hence it is a finite $p$-group [23]. This implies that $G_{n}$ is virtually free and so the Cayley graph of $G_{n}$ corresponding to the generators $t, a_{0}$ is $\delta_{n}$-hyperbolic for some $\delta_{n}$.

Now we are going to explain that choosing $c_{n+1} \gg c_{n}$ we can always ensure the condition $\delta_{n}=o\left(t_{n}\right)$ from Theorem 3.3. By the results of Higman [24], $C_{n}$ is residually (finite $p$-group), and so it is residually nilpotent.

Now observe that for every $c=c_{n+1}$, the image of $U_{n+1}=U_{n+1}(c)$ in $C_{n}$ belongs to the $(c+1)$-st term $\gamma_{c+1}\left(C_{n}\right)$ of the lower central series of $C_{n}$ and since $C_{n}$ is residually nilpotent we can make the natural homomorphism $C_{n} \rightarrow C_{n} / \gamma_{c+1}\left(C_{n}\right)$ (and, hence, the homomorphism $C_{n} \rightarrow C_{n+1}$ ) injective on any given finite subset by 
choosing big enough $c$. Hence the homomorphism $G_{n} \rightarrow G_{n+1}$ can be made injective on the ball of radius $t_{n}=\exp \left(\delta_{n}\right)$, for example.

Finally we note that the set of relations of the direct limit group $G$ coincides with the set of relation of the group $G(p, \mathbf{c})$ which is not virtually cyclic by Lemma 3.23 (c). This completes the proof.

Combining Theorem 3.3 and Lemma 3.24, we obtain the following.

Theorem 3.25 There exists a finitely generated elementary amenable group $G$ and a scaling sequence $d=\left(d_{n}\right)$ such that $G$ is not virtually cyclic and for any ultrafilter $\omega$, the asymptotic cone $\operatorname{Con}^{\omega}(G, d)$ is an $\mathbb{R}$-tree.

Note that $G(p, \mathbf{c})$ from Lemma 3.23 is clearly not finitely presented (because it is not virtually free and is a direct limit of virtually free groups). Hence it has a non-simply connected asymptotic cone [11]. Thus we obtain the following corollary.

Corollary 3.26 There is a finitely generated elementary amenable group having at least two nonhomeomorphic asymptotic cones.

\section{Graded small cancellation and circle-tree asymptotic cones}

Recall the definition of circle-trees.

Definition 4.1 We say that a metric space $X$ is a circle-tree, if $X$ is tree graded with respect to a collection of circles (with the standard length metric) whose radii are uniformly bounded from below and from above by positive constants. In particular, every circle-tree is locally isometric to an $\mathbb{R}$-tree.

Note that by Lemma 3.1, any group having a circle-tree asymptotic cone is lacunary hyperbolic. In this Section, we shall show that the class of groups all of whose asymptotic cones are trees or circle-trees is very large and contains all groups given by presentations satisfying certain small cancellation conditions. As a consequence, this class contains groups all of whose proper subgroups are cyclic or finite. 


\subsection{The Greendlinger Lemma for small cancellation presentations over hyperbolic groups}

Let $H$ be a group generated by a finite set $S$. We will consider quotient groups of $H$ as groups given by presentations over $H$ (ie presentations including all relations of $H$ plus some extra relations). Our goal is to generalize Definition 3.6 for such presentations.

We start with a definition of a piece. In what follows we write $U \equiv V$ for two words $U$ and $V$ is some alphabet to express letter-by-letter equality.

Definition 4.2 Let $H$ be a group generated by a set $S$. Let $\mathcal{R}$ be a symmetrized set of reduced words in $S^{ \pm 1}$. For $\varepsilon>0$, a subword $U$ of a word $R \in \mathcal{R}$ is called a $\varepsilon$-piece if there exists a word $R^{\prime} \in \mathcal{R}$ such that

(1) $R \equiv U V, R^{\prime} \equiv U^{\prime} V^{\prime}$, for some $V, U^{\prime}, V^{\prime}$;

(2) $U^{\prime}=Y U Z$ in $H$ for some words $Y, Z$ such that $\max \{|Y|,|Z|\} \leq \varepsilon$;

(3) $Y R Y^{-1} \neq R^{\prime}$ in the group $H$.

Note that if $U$ is an $\varepsilon$-piece, then $U^{\prime}$ is an $\varepsilon$-piece as well.

Recall that a word $W$ in the alphabet $S^{ \pm 1}$ is called $(\lambda, c)$-quasigeodesic (respectively geodesic) in $H$ if any path in $\Gamma(H, S)$ labeled by $W$ is $(\lambda, c)$-quasigeodesic (respectively geodesic).

Definition 4.3 Let $\varepsilon \geq 0, \mu \in(0,1)$, and $\rho>0$. We say that a symmetrized set $\mathcal{R}$ of words over the alphabet $S^{ \pm 1}$ satisfies the condition $C(\varepsilon, \mu, \rho)$ for the group $H$, if

$\left(C_{1}\right)$ all words from $\mathcal{R}$ are geodesic in $H$;

$\left(C_{2}\right) \quad|R| \geq \rho$ for any $R \in \mathcal{R}$;

$\left(C_{3}\right)$ the length of any $\varepsilon$-piece contained in any word $R \in \mathcal{R}$ is smaller than $\mu|R|$.

Suppose now that $H$ is a group defined by

$$
H=\langle S \mid \mathcal{O}\rangle,
$$

where $\mathcal{O}$ is the set of all relators (not only defining) of $H$. Given a symmetrized set of words $\mathcal{R}$, we consider the quotient group

$$
H_{1}=\langle H \mid \mathcal{R}\rangle=\langle S \mid \mathcal{O} \cup \mathcal{R}\rangle .
$$

A cell in a van Kampen diagram over $(8)$ is called an $\mathcal{R}$-cell (respectively, an $\mathcal{O}$-cell) if its boundary label is a word from $\mathcal{R}$ (respectively, $\mathcal{O}$ ). We always consider van 
Kampen diagrams over (8) up to some natural elementary transformations. For example we do not distinguish diagrams if one can be obtained from the other by joining two distinct $\mathcal{O}$-cells having a common edge or by the inverse transformation, etc. (see Ol'shanskii [42, Section 5] for details).

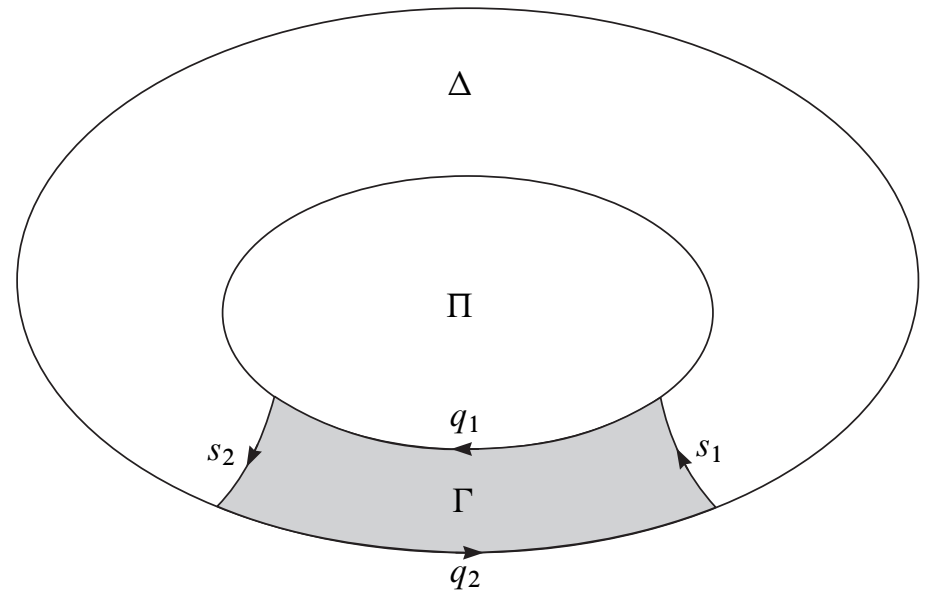

Figure 2: A contiguity subdiagram

Let $\Delta$ be a van Kampen diagram over (8), $q$ a subpath of its boundary $\partial \Delta, \Pi, \Pi^{\prime}$ some $\mathcal{R}$-cells of $\Delta$. Suppose that there is a simple closed path $p=s_{1} q_{1} s_{2} q_{2}$ in $\Delta$, where $q_{1}$ (respectively $q_{2}$ ) is a subpath of the boundary $\partial \Pi$ (respectively $q$ or $\partial \Pi^{\prime}$ ) and $\max \left\{\left|s_{1}\right|,\left|s_{2}\right|\right\} \leq \varepsilon$ for some constant $\varepsilon$. By $\Gamma$ we denote the subdiagram of $\Delta$ bounded by $p$. If $\Gamma$ contains no $\mathcal{R}$-cells, we say that $\Gamma$ is an $\varepsilon$-contiguity subdiagram of $\Pi$ to the part $q$ of $\partial \Delta$ or $\Pi^{\prime}$ respectively (Figure 2). The subpaths $q_{1}$ and $q_{2}$ are called contiguity arcs of $\Gamma$ and the ratio $\left|q_{1}\right| /|\partial \Pi|$ is called the contiguity degree of $\Pi$ to $\partial \Delta$ (or $\left.\Pi^{\prime}\right)$ and is denoted by $(\Pi, \Gamma, \partial \Delta)\left(\right.$ or $\left.\left(\Pi, \Gamma, \Pi^{\prime}\right)\right)$.

The following easy observation will often be useful.

Lemma 4.4 Suppose that the group $H$ is hyperbolic. Let $\mathcal{R}$ be a set of geodesic in $H$ words, $\Delta$ a diagram over (8), and $q$ a subpath of $\partial \Delta$ whose label is geodesic in $H_{1}$. Then for any $\varepsilon \geq 0$, no $\mathcal{R}$-cell $\Pi$ in $\Delta$ have an $\varepsilon$-contiguity subdiagram $\Gamma$ to $q$ such that $(\Pi, \Gamma, q)>1 / 2+2 \varepsilon /|\partial \Pi|$.

Proof Let $\Gamma$ be an $\varepsilon$-contiguity subdiagram of an $\mathcal{R}$-cell $\Pi$ to $q, \partial \Gamma=s_{1} q_{1} s_{2} q_{2}$, where $q_{2}$ is a subpath of $q$ and $\partial \Pi=q_{1} r$. Let also $\gamma$ denote the contiguity degree $(\Pi, \Gamma, q)$. Then we have $\left|q_{2}\right| \geq\left|q_{1}\right|-\left|s_{1}\right|-\left|s_{2}\right|=\gamma|\partial \Pi|-2 \varepsilon$ since $\operatorname{Lab}\left(q_{1}\right)$ is geodesic in $H$. On the other hand, $\left|q_{2}\right| \leq|r|+\left|s_{1}\right|+\left|s_{2}\right| \leq(1-\gamma)|\partial \Pi|+2 \varepsilon$ as $\operatorname{Lab}\left(q_{2}\right)$ is geodesic in $H$. These two inequalities yield $\gamma \leq 1 / 2+2 \varepsilon /|\partial \Pi|$. 
Given a van Kampen diagram $\Delta$ over (8), we call a combinatorial map from the 1 -skeleton $\mathrm{Sk}^{(1)}(\Delta)$ to the Cayley graph $\Gamma\left(H_{1}, S\right)$ natural if it preserves labels and orientation of edges. The following easy observation will be useful.

Lemma 4.5 Suppose that $H$ is hyperbolic. Let $\mathcal{R}$ be a symmetrized set of words in $S^{ \pm 1}$ satisfying the condition $C(\varepsilon, \mu, \rho)$ for some $\varepsilon \geq 0, \mu \in(0,1), \rho>0$. Suppose that $\Pi, \Pi^{\prime}$ are two $\mathcal{R}$-cells in a diagram $\Delta$ over $(8)$ and $\Gamma$ is an $\varepsilon$-contiguity subdiagram of $\Pi^{\prime}$ to $\Pi$ such that $\gamma=\left(\Pi^{\prime}, \Gamma, \Pi\right) \geq \mu$. Then

$$
|| \partial \Pi|-| \partial \Pi^{\prime}|| \leq 2 \varepsilon
$$

and for any natural map $\phi: \mathrm{Sk}^{(1)}(\Delta) \rightarrow \Gamma\left(H_{1}, S\right)$, the Hausdorff distance between $\phi(\partial \Pi)$ and $\phi\left(\partial \Pi^{\prime}\right)$ does not exceed $2 \varepsilon+2 \delta$.

Proof Let $\partial \Pi=u v,\left(\partial \Pi^{\prime}\right)^{-1}=u^{\prime} v^{\prime}$, and $\partial \Gamma=y u z\left(u^{\prime}\right)^{-1}$. Since $\operatorname{Lab}(y u z)=$ $\operatorname{Lab}\left(u^{\prime}\right)$ in $H$, we have

$$
\operatorname{Lab}(y) \operatorname{Lab}(u v) \operatorname{Lab}(y)^{-1}=\operatorname{Lab}\left(u^{\prime} v^{\prime}\right)
$$

in $H$ by the $C(\varepsilon, \mu, \rho)$-condition. As labels of $\partial \Pi$ and $\partial \Pi^{\prime}$ are geodesic in $H,(10)$ implies (9).

Further let $Q=a b c d$ be a quadrangle in $\Gamma(H, S)$ such that $a, b, c$, and $d$ are labeled by $\operatorname{Lab}(y), \operatorname{Lab}(u v), \operatorname{Lab}(y)^{-1}$, and $\left(\operatorname{Lab}\left(u^{\prime} v^{\prime}\right)\right)^{-1}$, respectively. By the first assertion of Lemma 2.9, $b$ and $d$ belong to the closed $(\varepsilon+2 \delta)$-neighborhoods of each other. To finish the proof it remains to note that the map $\Gamma(H, S) \rightarrow \Gamma\left(H_{1}, S\right)$ induced by the homomorphism $H \rightarrow H_{1}$ does not increase the distance.

We call a (disc) van Kampen diagram over (8) minimal if it has minimal number of $\mathcal{R}$-cells among all disc diagrams with the same boundary label. The first part of the following result is an analog of the Greendlinger Lemma 3.7 for presentations over hyperbolic groups.

Lemma 4.6 Suppose that $H$ is a $\delta$-hyperbolic group having presentation $\langle S \mid \mathcal{O}\rangle$ as in (7), $\varepsilon \geq 2 \delta, 0<\mu \leq 0.01$, and $\rho$ is large enough (it suffices to choose $\rho>10^{6} \varepsilon / \mu$ ). Let $H_{1}$ be given by a presentation

$$
H_{1}=\langle H \mid \mathcal{R}\rangle=\langle S \mid \mathcal{O} \cup \mathcal{R}\rangle
$$

as in (8) where $\mathcal{R}$ is a finite symmetrized set of words in $S^{ \pm 1}$ satisfying the $C(\varepsilon, \mu, \rho)$ condition. Then the following statements hold. 
(1) Let $\Delta$ be a minimal disc diagram over (8). Suppose that $\partial \Delta=q^{1} \cdots q^{t}$, where the labels of $q^{1}, \ldots, q^{t}$ are geodesic in $H$ and $t \leq 12$. Then, provided $\Delta$ has an $\mathcal{R}$-cell, there exists an $\mathcal{R}$-cell $\Pi$ in $\Delta$ and disjoint $\varepsilon$-contiguity subdiagrams $\Gamma_{1}, \ldots, \Gamma_{t}$ (some of them may be absent) of $\Pi$ to $q^{1}, \ldots, q^{t}$ respectively such that

$$
\left(\Pi, \Gamma_{1}, q^{1}\right)+\cdots+\left(\Pi, \Gamma_{t}, q^{t}\right)>1-23 \mu .
$$

(2) $H_{1}$ is a $\delta_{1}$-hyperbolic group with $\delta_{1} \leq 4 r$ where $r=\max \{|R| \mid R \in \mathcal{R}\}$.

Proof The first part of the lemma is essentially a special case of [42, Lemma 6.6] where the parameters $\lambda$ and $c$ of the quasigeodesity of the defining words are equal to 1 and 0 , respectively because the words in $\mathcal{R}$ are geodesic in $H$. The minor corrections in the argument of [42] leading to this special case are the following.

We replace $t \leq 4$ by $t \leq 12$. Note that the proof from [42] works even in the case $t \leq k$, for any fixed $k$, but then we should replace 23 by $C=C(k)$ in the formulation of the lemma.

As in the proof from [42], we need to consider geodesic quadrangles $s_{1} q_{1} s_{2} q_{2}$ with "short" sides $s_{1}, s_{2}$ and "long" sides $q_{1}, q_{2}$. Then if a point $o \in q_{1}$ is far from the ends of $q_{1}$, say, $\min \left(\operatorname{dist}\left(o, q_{-}\right), \operatorname{dist}\left(o, q_{+}\right)\right) \geq \max \left(\left|s_{1}\right|,\left|s_{2}\right|\right)+2 \delta$ then in [42], it is proved that the distance from $o$ to $q_{2}$ is bounded from above by $c_{1}=13 \delta$. In our case, by Lemma 2.9, we can take $c_{1}=2 \delta$.

Finally, in [42, Lemma 6.2], we can replace the upper estimate $n \sqrt{\rho}$ by $n \mu \rho$ (this is possible because $\rho$ is large enough).

The proof of the second statement of the lemma is divided into two steps.

Step 1 First we consider a minimal diagram $\Gamma$ over $H_{1}$ whose contour contains a subpath $p q$ where segments $p$ and $q$ are geodesic in $\Gamma$. Assume that there is an $\mathcal{R}$-cell $\Pi$ with two contiguity subdiagrams $\Gamma_{p}$ and $\Gamma_{q}$ to $p$ and $q$, respectively, and with contiguity arcs $v_{p} \subseteq p$ and $v_{q} \subseteq q$, such that $\left(v_{p}\right)_{-}=p_{-}$and $\left(v_{q}\right)_{+}=q_{+}$. We claim that the Hausdorff distance between the images $\bar{p}$ and $\bar{q}$ of $p$ and $q$ in the Cayley graph of $H_{1}$ does not exceed $2 r$. We may assume that the boundary path of $\Gamma$ is the product of $p q$, an arc of $\Pi$, and two side arcs of $\Gamma_{p}$ and $\Gamma_{q}$ (see Figure 3 ).

Let $v_{p} x u y$ be the boundary path of $\Gamma_{p}$ where $u$ is the contiguity arc of $\Gamma_{p}$ lying on $\partial \Pi$. Since $v_{p}$ is geodesic we have $\left|v_{p}\right| \leq|x|+|y|+|u|<2 \varepsilon+r$. Therefore, using $\Pi$ and the contiguity subdiagrams, we can connect every point of $v_{p}$ (of $v_{q}$ ) to $q$ (to $p$ ) with a path of length $\leq\left|v_{p}\right| / 2+2 \varepsilon+r<2 r$ since, according to the first statement of the lemma, $\rho$ is chosen much greater than $\varepsilon$. 


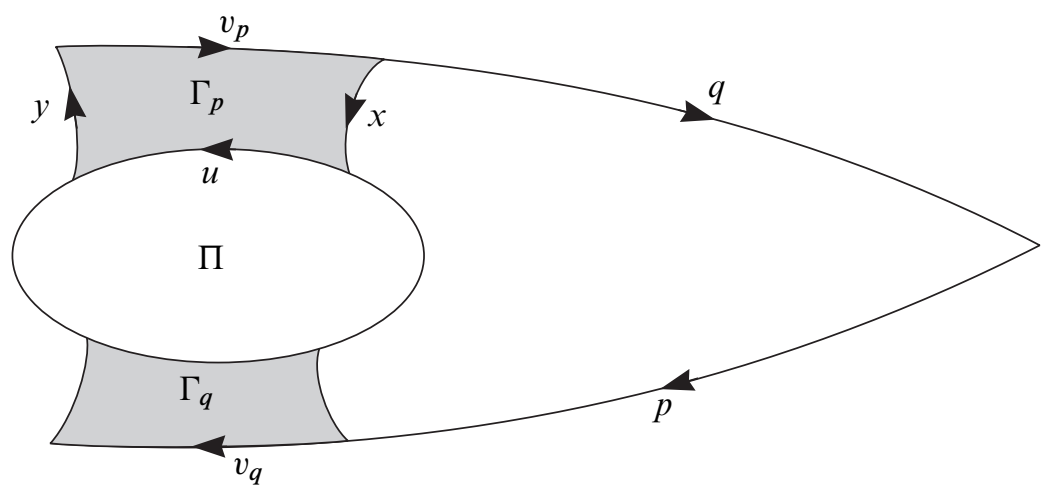

Figure 3: The diagram $\Gamma$

Then we consider a maximal set of $\mathcal{R}$-cells $\Pi, \Pi^{\prime}, \Pi^{\prime \prime}, \ldots$ in $\Gamma$ having disjoint contiguity subdiagrams $\Gamma_{p}, \Gamma_{q}, \Gamma_{p}^{\prime}, \Gamma_{q}^{\prime}, \ldots$ to both $p$ and $q$. After suitable enumeration, they provide us with decompositions $p=v_{p} w_{p} v_{p}^{\prime} w_{p}^{\prime} \ldots$ and $q=\ldots w_{q}^{\prime} v_{q}^{\prime} w_{q} v_{q}$ where $v_{p}, v_{p}^{\prime}, v_{p}^{\prime \prime}, \ldots$ and $v_{q}, v_{q}^{\prime}, v_{q}^{\prime \prime}, \ldots$ are the contiguity arcs of the above contiguity subdiagrams. As in the previous paragraph, we have that the distance between every point of $v_{p}^{\prime}, v_{p}^{\prime \prime}, \ldots$ ( of $v_{q}^{\prime}, v_{q}^{\prime \prime}, \ldots$ ) and $q$ (and $p$ ) is less than $2 r$. Thus it suffices to obtain the same estimate for the distance between a point of one of $w_{p}, w_{p}^{\prime}, \ldots$ and $q$. (More precisely, it suffices to do this with the images $\bar{w}_{p}, \ldots, \bar{q}$ of these paths in the Cayley graph of $H_{1}$.)

For example, $w_{p}^{\prime}$ is a section of a loop $w_{p}^{\prime} a b c w_{q}^{\prime} d e f$ where $b$ and $e$ are arcs of $\Pi^{\prime}$ and $\Pi^{\prime \prime}$, respectively, and $a, c, d, f$ are geodesics of length at most $\varepsilon$. Denote by $\Xi$ the subdiagram bounded by this octagon. If $\Xi$ is a diagram over $H$, then by Lemma 2.9, every point of $\bar{w}_{p}^{\prime}$ is at distance at most $6 \delta$ from the union of the remaining 7 sides. Since $\max (|b|,|e|) \leq r$, we have that the distance between a point of $w_{p}^{\prime}$ and $w_{q}^{\prime}$ is at most $6 \delta+2 \varepsilon+r<2 r$ since the parameter $\rho$ is chosen so that $\rho>6 \delta+2 \varepsilon$.

Thus to complete Step 1, it suffices to show that $\Xi$ contains no $\mathcal{R}$-cells. Arguing by contradiction, we have an $\mathcal{R}$-cell $\pi$ and its contiguity subdiagrams $\Gamma_{1}, \ldots, \Gamma_{8}$ (some of them may be absent) to $w_{p}^{\prime}, a, \ldots, f$, respectively, with

$$
\left(\pi, \Gamma_{1}, w_{p}^{\prime}\right)+\cdots+\left(\pi, \Gamma_{8}, f\right)>1-23 \mu
$$

by the first assertion of the lemma. Here the contiguity degree to $b$ and $e$ are less than $\mu$ by the $C(\varepsilon, \mu, \rho)$-condition. Since the lengths of $a, c, d, f$ are less than $\varepsilon \ll \rho$, the contiguity degree of $\pi$ to each of these four boundary sections of $\Xi$ is less than $\mu / 2$. (The accurate proof of the latter inequality is given in [42, Lemma 6.5(a)].) Hence $\left(\pi, \Gamma_{1}, w_{p}^{\prime}\right)+\left(\pi, \Gamma_{5}, w_{q}^{\prime}\right)>1-27 \mu$. 
If, for example, $\Gamma_{5}$ is absent, then $w_{p}^{\prime}$ is homotopic in $\Gamma$ to the path $x^{\prime} z y^{\prime}$, where $x^{\prime}$ and $y^{\prime}$ are side arcs of contiguity subdiagram and $z$ an arc on the boundary $\partial \pi$ with length $<27 \mu|\partial \pi|$. Since $w_{p}^{\prime}$ is geodesic, we have $\left|w_{p}^{\prime}\right|<2 \varepsilon+27 \mu|\partial \pi|$. On the other hand, $w_{p}^{\prime}$ is homotopic to $x^{\prime} u^{\prime} y^{\prime}$ in the diagram $\Gamma_{p}$ over $H$ where $u^{\prime}$ is the arc of $\pi$ of length at least $(1-27 \mu)|\partial \pi|$. Therefore $\left|w_{p}^{\prime}\right|+2 \varepsilon>(1-27 \mu)|\partial \pi|$. Since $|\partial \pi| \geq \rho$ we obtain $(1-54 \mu) \rho<4 \varepsilon$ that contradicts $\rho>10^{6} \mu^{-1} \varepsilon$.

Thus both $\Gamma_{1}$ and $\Gamma_{5}$ are present, and so the cell $\pi$ can be added to the set $\Pi, \Pi^{\prime}, \ldots$ contrary the maximality. This contradiction completes Step 1.

Step 2 Assume that $\delta_{1}>4 r$. Then, by Rips' definitions of hyperbolicity (see Gromov $[20,6.6])$ there exists a geodesic triangle $x y z$ in the Cayley graph of $H_{1}$ such that

( for arbitrary three points $o_{1}, o_{2}, o_{3}$ chosen on the sides $x, y$, and $z$, respectively, we have

$$
\max \left(\operatorname{dist}\left(o_{1}, o_{2}\right), \operatorname{dist}\left(o_{2}, o_{3}\right), \operatorname{dist}\left(o_{3}, o_{1}\right)\right) \geq \delta_{1}>4 r .
$$

Let $\Delta$ be a minimal diagram over $H_{1}$ corresponding to the triangle. We preserve the notation $x y z$ for the boundary of $\Delta$. A subdiagram $\Gamma$ is said to be an $x y$-corner of $\Delta$ if for some subpaths $p$ of $x$ and $q$ of $y$ such that $p_{+}=q_{-}$(ie, $p q$ is a subpath of $x y$ ), it contains a cell $\Pi$ with two contiguity subdiagrams $\Gamma_{p}$ and $\Gamma_{q}$ satisfying the conditions of Step 1, and it is bounded by $p, q, \Pi, \Gamma_{p}$ and $\Gamma_{q}$ as in the first paragraph of Step 1. The $x y$-corner is called maximal, if the sum $|p|+|q|$ is maximal. By definition, it consists of the single vertex $x_{+}=y_{-}$if there exists no $\Pi$ as above. Similarly we define $y z$ - and $z x$-corners.

In this notation, we suppose that the $x y$-corner $\Gamma=\Gamma_{x y}$ is maximal, $x$ contains the subsegment $p$, and let $p^{\prime}$ be a similar segment of a maximal $z x$-corner $\Gamma_{z x}$ where $p^{\prime}$ also lies on $x$. If $p$ and $p^{\prime}$ together cover $x$, then every point of $x$ belongs to the $2 r$-neighborhood of the union of two other sides of the triangle $x y z$. This implies that there is a point $o_{1}$ on $x$ and two points $o_{2}$ and $o_{3}$ on $y$ and $z$, respectively, such that $\max \left(\operatorname{dist}\left(o_{1}, o_{2}\right), \operatorname{dist}\left(o_{1}, o_{3}\right)\right) \leq 2 r$, and this contradicts Condition $(\star)$. Hence $p$ and $p^{\prime}$ must be disjoint. It follows that all three maximal corners $\Gamma_{x y}, \Gamma_{y z}$ and $\Gamma_{z x}$ can be chosen pairwise disjoint(see Figure 4).

Now consider the diagram $\Delta^{\prime}$ obtained from $\Delta$ by cutting off these three corners. The contour of $\Delta^{\prime}$ is of the form $x^{\prime} a y^{\prime} b z^{\prime} c$ where $x^{\prime}, y^{\prime}$, and $z^{\prime}$ belong in $x, y$, and $z$, respectively. In turn, $a=a_{1} a_{2} a_{3}$ where $a_{2}$ is an arc of the cell $\Pi$ from the definition of corner, and $a_{1}, a_{3}$ are side arcs of $\Gamma_{p}$ and $\Gamma_{q}$. Similarly, $b=b_{1} b_{2} b_{3}$ and $c=c_{1} c_{2} c_{3}$. Thus we have a decomposition of $\partial \Delta^{\prime}$ in 12 sections. Note that $\sum_{i=1}^{3}\left(\left|a_{i}\right|+\left|b_{i}\right|+\left|c_{i}\right|\right) \leq 6 \varepsilon+3 r$. 


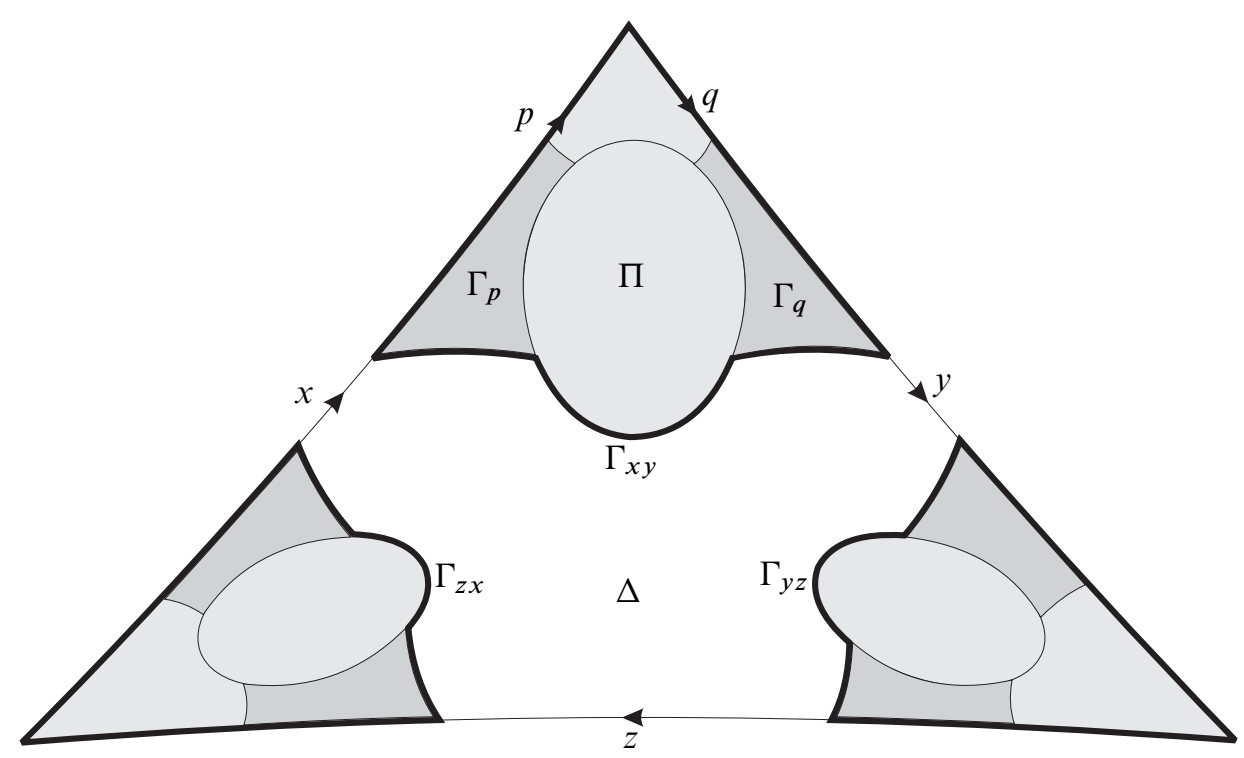

Figure 4: The corners $\Gamma_{x y}, \Gamma_{y z}$ and $\Gamma_{z x}$ in $\Delta$

If $\Delta^{\prime}$ is a diagram over $H$, then by Lemma $2.9, x^{\prime}$ belongs to the $10 \delta$-neighborhood of the union of the remaining 11 sides of the 12-gon, and so $x^{\prime}$ belongs to the $(10 \delta+6 \varepsilon+3 r)$-neighborhood of $y \cup z$. Since $10 \delta+6 \varepsilon<\rho \leq r$, every point of $x^{\prime}$ belongs to the $4 r$-neighborhood of $y \cup z$. Recall that every point of $x \backslash x^{\prime}$ is in $p \cup p^{\prime}$ and belongs in the $2 r$-neighborhood of $y \cup z$. Similarly, every point of $y$ or $z$ belongs in $4 r$-neighborhood of the union of two other sides, which contradicts to the choice of the triangle $x y z$.

Then we may assume that $\Delta^{\prime}$ has an $\mathcal{R}$-cell, and so, by the first assertion of the lemma, it has a cell $\pi$ with contiguity subdiagrams $\Gamma_{x^{\prime}}, \Gamma_{a_{1}}, \ldots$ to the 12 sections, such that the sum of their contiguity degrees is greater than $1-23 \mu$. As at the Step 1, the sum of contiguity degrees for all $a_{i}, b_{i}, c_{i}(i=1,2,3)$ is less than $6 \mu$. So the sum of the contiguity degrees to $x^{\prime}, y^{\prime}$, and $z^{\prime}$ is greater than $1-29 \mu$. It is impossible that two of these three subdiagrams be absent because the degree of contiguity of $\pi$ to a geodesic section cannot be as high as $1-29 \mu$ by the argument of Step 1 with coefficient 27 replaced by 29 .

Therefore we may assume that there are contiguity subdiagrams of $\pi$ to both $x^{\prime}$ and $y^{\prime}$. But this contradicts the maximality of the corner $\Gamma_{x y}$, and the lemma is completely proved. 


\subsection{Comparing various small cancellation conditions}

The purpose of this section is to compare the small cancellation condition introduced in Section 4.1 with the classical small cancellation condition.

Lemma 4.7 Let $R_{1}, R_{2}, \ldots$ be an infinite set of distinct cyclically reduced words in a finite alphabet $S, \mathcal{R}_{i}$ the set of all cyclic shifts of $R_{i}^{ \pm 1}$, and $\mathcal{R}=\bigcup_{i=1}^{\infty} \mathcal{R}_{i}$. Assume that

$\left(S C_{1}\right)$ the set $\mathcal{R}$ satisfies $C^{\prime}(\lambda)$ for some $\lambda \leq 1 / 10$;

$\left(S C_{2}\right)$ the set $\mathcal{R}_{n}(n=1,2, \ldots)$ satisfies $C^{\prime}\left(\lambda_{n}\right)$ where $\lambda_{n} \rightarrow 0$.

Let also $H=\left\langle S \mid R_{i_{1}}, \ldots, R_{i_{j}}\right\rangle$ for some $i_{1}<\cdots<i_{j}$. Then, for every $\mu>0, \varepsilon \geq 0$, and $\rho>0$, there is $n>i_{j}$ such that the set $\mathcal{R}_{n}$ satisfies the $C(\varepsilon, \mu, \rho)-$ condition over $H$.

Proof We first prove that any subword $V$ of a cyclic shift of $R_{n}^{ \pm 1}$ is geodesic in the group $H$. Let $U$ be a geodesic word equal to $V$ in $H$. Notice that $V$ has no subword $W$ which is also a subword of one of the words $R$ from $\mathcal{T}=\mathcal{R}_{i_{1}} \cup \cdots \cup \mathcal{R}_{i_{j}}$ with $|V| \geq \lambda|R|$ by Condition (SC $C_{1}$ ). Then by Lemma 3.7, either $U \equiv V$, and we are done, or there is a subword $W$ of $U$ which is also a prefix of a word $R$ from $\mathcal{T}$ with $|W|>(1-3 \lambda-2 \lambda)|R| \geq|R| / 2$. Therefore the subword $W$ is equal in $H$ to a shorter word $T^{-1}$, where $R \equiv W T=1$ in $H$. But then $U$ is not geodesic, a contradiction.

Now, it remains to show that a word from $\mathcal{R}_{n}$ has no $\varepsilon$-pieces of length at least $\mu\left|R_{n}\right|$ if $n$ is large enough. We may further assume by $\left(S C_{2}\right)$ that $\lambda_{n}<\mu / 2$ and $\left|R_{n}\right|>\mu^{-1} \max \left(\left|R_{i_{j}}\right|, 4 \varepsilon\right)$. (Observe that $\left(S C_{2}\right)$ implies $\left|R_{n}\right| \rightarrow \infty$ and $n \rightarrow \infty$.)

Assume that $R, R^{\prime} \in \mathcal{R}_{n}, R \equiv U V, R^{\prime} \equiv U^{\prime} V^{\prime},|U| \geq \mu\left|R_{n}\right|$, and $U^{\prime}=Y U Z$ in $H$ for some geodesic in $H$ words $Y, Z$ with $\max (|Y|,|Z|) \leq \varepsilon$. We must prove that $Y R Y^{-1}=R^{\prime}$ in $H$.

Consider a diagram $\Delta$ over $H$ with boundary path $p_{1} q_{1} p_{2} q_{2}$, where $\operatorname{Lab}\left(p_{1}\right) \equiv Y$, $\operatorname{Lab}\left(q_{1}\right) \equiv U, \operatorname{Lab}\left(q_{2}\right) \equiv Z$, and $\operatorname{Lab}\left(p_{2}\right) \equiv\left(U^{\prime}\right)^{-1}$.

If $\Delta$ has no cells then there is a common subpath $t$ of $q_{1}$ and $q_{2}^{-1}$ with $|t| \geq \lambda_{n}\left|R_{n}\right|$ because

$$
\left|p_{1}\right|+\left|p_{2}\right| \leq 2 \varepsilon<\mu\left|R_{n}\right| / 2<\left(\mu-\lambda_{n}\right)\left|R_{n}\right|,
$$

and every edge of $q_{1}$ belong to the path $p_{2} q_{2} p_{1}$. From the small cancellation condition $\left(S C_{1}\right)$, we see that $T \equiv \operatorname{Lab}(t)$ is a prefix of a unique word $R_{0}$ from $\mathcal{R}_{n}$. Since $U \equiv X_{1} T X_{2}$ and $U^{\prime} \equiv X_{3} T X_{4}$ for some words $X_{1}, X_{2}, X_{3}, X_{4}$, we obtain that the conjugates $X_{1}^{-1} R X_{1}$ and $X_{3}^{-1} R^{\prime} X_{3}$ are both freely equal to $R_{0}$, ie 
$R=X_{1} X_{3}^{-1} R^{\prime}\left(X_{1} X_{3}^{-1}\right)^{-1}$. Since $Y X_{1} X_{3}^{-1}$ is a label of a closed path in $\Delta$, we have $Y R Y^{-1}=\left(Y X_{1} X_{3}^{-1}\right) R^{\prime}\left(Y X_{1} X_{3}^{-1}\right)^{-1}=R^{\prime}$ in $H$, as required.

Now assume by contradiction that $\Delta$ has a cell. No cell $\Pi$ has a boundary arc with length $>|\partial \Pi| / 2$ contained in $p_{1}$ or in $p_{2}$ because these segments are geodesic. Since $q_{1}$ is a geodesic path by (1), no cell $\Pi$ can have vertices in both $p_{1}$ and $p_{2}$ because otherwise

$$
\mu\left|R_{n}\right| \leq\left|q_{1}\right| \leq\left|p_{1}\right|+|\partial \Pi| / 2+\left|p_{2}\right| \leq 2 \varepsilon+\left|R_{i_{j}}\right| / 2 \leq \max \left(\left|R_{i_{j}}\right|, 4 \varepsilon\right)
$$

contrary to the choice of $n$. Then it follows from Lemma 3.7, that there is a cell $\Pi$ in $\Delta$, whose boundary has a common subpath $t$ with either $q_{1}$ or $q_{2}$, where $|t|>\frac{1}{2}(1-3 \lambda-1 / 2)|\partial \Pi| \geq \lambda|\partial \Pi|$. But this contradicts Condition $\left(S C_{1}\right)$ because $n \notin\left\{i_{1}, \ldots, i_{j}\right\}$.

Remark 4.8 Infinite sets of words in the alphabet $\{a, b\}$ satisfying $\left(S C_{1}\right)$ and $\left(S C_{2}\right)$ were constructed in various places (see, for example, Erschler and Osin [15]). Moreover, one can find such sets satisfying $\left(S C_{1}\right)$ for arbitrary small $\lambda>0$.

Our next goal is to relate $C(\varepsilon, \mu, \rho)$ to the small cancellation condition $C(\varepsilon, \mu, \lambda, c, \rho)$ introduced in [42]. Essentially, this condition is obtained from $C(\varepsilon, \mu, \rho)$ by replacing the word "geodesic" by "quasigeodesic"; the additional parameters are the quasigeodesic parameters. We shall show that any $C(\varepsilon, \mu, \lambda, c, \rho)$-presentation can be transformed into a $C\left(\varepsilon^{\prime}, \mu^{\prime}, \rho^{\prime}\right)$-presentation of the same group for suitable $\varepsilon^{\prime}, \mu^{\prime}, \rho^{\prime}$.

Definition 4.9 Let $H$ be a hyperbolic group generated by a finite set $S$. A symmetrized set $\mathcal{R}$ of words in $S^{ \pm 1}$ satisfies the condition $C(\varepsilon, \mu, \lambda, c, \rho)$ for some constants $\varepsilon>0, \mu \in(0,1), \lambda \in(0,1], c \geq 0, \rho>0$, if all words in $\mathcal{R}$ are $(\lambda, c)-$ quasigeodesic and conditions $\left(C_{2}\right),\left(C_{3}\right)$ from Definition 4.3 hold. Thus $C(\varepsilon, \mu, \rho)$ is equivalent to $C(\varepsilon, 1,0, \mu, \rho)$.

In the two lemmas below the following notation is used. Let $\mathcal{R}_{0}$ be a set of words in $S^{ \pm 1}, \mathcal{R}_{0}^{\prime}$ the set obtained from $\mathcal{R}_{0}$ by replacing each $R \in \mathcal{R}_{0}$ with a shortest word $R^{\prime}$ such that $R$ and $R^{\prime}$ are conjugate in $H$. Denote by $\mathcal{R}$ (respectively $\mathcal{R}^{\prime}$ ) the set of all cyclic shifts of words from $\mathcal{R}_{0}^{ \pm 1}$ (respectively $\left(\mathcal{R}_{0}^{\prime}\right)^{ \pm 1}$ ). Note that all words in $\mathcal{R}^{\prime}$ are geodesic in $H$.

Given $\lambda>0, c \geq 0$, we define

$$
\kappa=8 \delta+4 \theta,
$$

where $\delta$ is the hyperbolicity constant of the Cayley graph $\Gamma(H, S)$, and $\theta=\theta(\lambda, c)$ is the constant from Lemma 2.8 . 
Lemma 4.10 Suppose that all words in $\mathcal{R}$ are $(\lambda, c)$-quasigeodesic in $H$ for some $\lambda, c$ and have lengths at least $(\kappa+c) / \lambda$. Then for every $W \in \mathcal{R}^{\prime}$, there is $R_{W} \in \mathcal{R}$ such that $W$ and $R_{W}$ are conjugate by an element of length at most $\kappa / 2$ in $H$.

Proof Any word $W \in \mathcal{R}^{\prime}$ is conjugate to some word $R \in \mathcal{R}^{ \pm 1}$ in $H$. Let $T_{W, R}$ denote a shortest word conjugating $W$ to $R$ in $H$. Let also $U$ and $S$ be cyclic shifts of $W$ and $R$ respectively such that

$$
|T(U, S)| \leq\left|T\left(U^{\prime}, S^{\prime}\right)\right|
$$

for any cyclic shifts $U^{\prime}$ and $S^{\prime}$ of $W$ and $R$.

There is a 4 -gon $a s b u^{-1}$ in $\Gamma(H, S)$ such that $\operatorname{Lab}(u) \equiv U, \operatorname{Lab}(s) \equiv S, \operatorname{Lab}(a) \equiv$ $\operatorname{Lab}\left(b^{-1}\right) \equiv T(U, S)$. Clearly $s$ is $(\lambda, c)$-quasigeodesic, and $a, u, b$ are geodesic. Moreover

$$
\operatorname{dist}(u, s) \geq|a|
$$

by (11). There are two cases to consider.

Case 1 If $|a| \leq 2 \delta+\theta$, then by Lemma 2.8 and Lemma 2.9, $u$ and $s$ belong to the closed $(4 \delta+2 \theta)$-neighborhoods of each other. Therefore, $W$ is conjugate to a cyclic shift of $R$ by a word of length at most $4 \delta+2 \theta$.

Case 2 Now assume that $|a|>2 \delta+\theta$. In particular, $\operatorname{dist}(u, s)>2 \delta+\theta$ by (12). Consider the middle point $m$ of $s$. Lemma 2.8 and Lemma 2.9 imply that $\operatorname{dist}(m, a \cup b) \leq 2 \delta+\theta$. For definiteness, assume that $\operatorname{dist}(m, a) \leq 2 \delta+\theta$. Let $z$ be the point on $a$ such that $\operatorname{dist}(m, a)=\operatorname{dist}(m, z)$. Then

$$
\operatorname{dist}\left(z, a_{-}\right) \geq \operatorname{dist}\left(m, a_{-}\right)-2 \delta-\theta \geq \operatorname{dist}(s, u)-2 \delta-\theta \geq|a|-2 \delta-\theta
$$

by (12). Therefore,

$$
\operatorname{dist}\left(z, s_{-}\right) \leq|a|-\operatorname{dist}\left(z, a_{-}\right) \leq 2 \delta+\theta \text {. }
$$

Consequently,

$$
\operatorname{dist}\left(m, s_{-}\right) \leq \operatorname{dist}(m, z)+\operatorname{dist}\left(z, s_{-}\right) \leq 2(2 \delta+\theta) .
$$

This means that a cyclic shift of $R$ represents an element of length at most $4(2 \delta+\theta)$ in $H$. Hence $|R| \leq \lambda^{-1}(8 \delta+4 \theta+c)$ that contradicts our assumption. Hence this case in impossible. 
Lemma 4.11 Suppose that $\mathcal{R}$ satisfies $C(\varepsilon, \mu, \lambda, c, \rho)$ for some $\varepsilon>0, \mu \in(0,1)$, $\lambda \in(0,1], c>0$, and

$$
\rho>2(c+3 \kappa) / \lambda
$$

Then $\mathcal{R}^{\prime}$ satisfies $C\left(\varepsilon^{\prime}, \mu^{\prime}, \rho^{\prime}\right)$ for

$$
\varepsilon^{\prime}=\varepsilon-2 \kappa, \quad \mu^{\prime}=2 \mu / \lambda, \quad \rho^{\prime}=\lambda \rho-c-\kappa .
$$

Proof By (13) and Lemma 4.10 for any word $W \in \mathcal{R}^{\prime}$, there is a word $R_{W} \in \mathcal{R}$ that is conjugate to $W$ by a word of length at most $\kappa / 2$ in $H$. In particular, this yields the last inequality in (14).

Suppose now that for some words $W_{1}, W_{2}$ of $\mathcal{R}^{\prime}$, we have $W_{1} \equiv U_{1} V_{1}, W_{2} \equiv U_{2} V_{2}$, and $U_{1}=Y U_{2} Z$ in $H$, where $|Y|,|Z| \leq \varepsilon^{\prime}$ and $\left|U_{1}\right| \geq \mu^{\prime}\left|W_{1}\right|$. Let $A_{1}, A_{2}$ be words of lengths at most $\kappa / 2$ such that

$$
R_{W_{i}}=A_{i} W_{i} A_{i}^{-1}, i=1,2
$$

in $H$. Using Lemma 2.8 and Lemma 2.9, we can find initial subwords $C_{i}$ of $R_{W_{i}}$, $i=1,2$, such that

$$
C_{i}=A_{i} U_{i} B_{i}, i=1,2
$$

in $H$, where

$$
\left|B_{i}\right| \leq \theta+2 \delta+\kappa / 2<\kappa .
$$

Thus $C_{1}=A_{1} Y A_{2}^{-1} C_{2} B_{2}^{-1} Z B_{1}$ in $H$. Note that

$$
\max \left\{\left|B_{2}^{-1} Z B_{1}\right|,\left|A_{1} Y A_{2}^{-1}\right|\right\} \leq \varepsilon^{\prime}+2 \kappa=\varepsilon .
$$

Now using subsequently (16), (17), (15), and (13) we obtain

$$
\begin{aligned}
\left|C_{1}\right| & \geq\left|U_{1}\right|-\left|A_{1}\right|-\left|B_{1}\right| \geq \mu^{\prime}\left|W_{1}\right|-2 \kappa \geq \mu^{\prime}\left(\lambda\left|R_{W_{1}}\right|-c-\kappa\right)-2 \kappa \\
& \geq 2 \mu\left|R_{W_{1}}\right|-2 \mu(c+\kappa) / \lambda-2 \kappa \geq \mu\left|R_{W_{1}}\right| .
\end{aligned}
$$

Hence

$$
R_{W_{1}}=\left(A_{1} Y A_{2}^{-1}\right) R_{W_{2}}\left(A_{1} Y A_{2}^{-1}\right)^{-1}
$$

in $H$ by the $C(\varepsilon, \lambda, c, \mu, \rho)$-condition. Combining this with (15), we obtain $W_{1}=$ $Y W_{2} Y^{-1}$. 


\subsection{Groups with circle-tree asymptotic cones}

In this section we define the graded small cancellation condition and prove that all asymptotic cones of any group given by a graded small cancellation presentation are circle-trees.

In the next two sections we show that many classical small cancellation groups as well as some "monsters" obtained by methods from [42] admit graded small cancellation presentations.

Definition 4.12 Let $\alpha, K$ be positive numbers. We say that the presentation

$$
\langle S \mid \mathcal{R}\rangle=\left\langle S \mid \bigcup_{i=0}^{\infty} \mathcal{R}_{i}\right\rangle
$$

of a group $G$ is a $\mathcal{Q}(\alpha, K)$-presentation if the following conditions hold for some sequences $\varepsilon=\left(\varepsilon_{n}\right), \mu=\left(\mu_{n}\right)$, and $\rho=\left(\rho_{n}\right)$ of positive real numbers $(n=1,2, \ldots)$.

$\left(\mathbf{Q}_{0}\right)$ The group $G_{0}=\left\langle S \mid \mathcal{R}_{0}\right\rangle$ is $\delta_{0}$-hyperbolic for some $\delta_{0}$.

$\left(\mathbf{Q}_{1}\right)$ For every $n \geq 1, \mathcal{R}_{n}$ satisfies $C\left(\varepsilon_{n}, \mu_{n}, \rho_{n}\right)$ over $G_{n-1}=\left\langle S \mid \bigcup_{i=0}^{n-1} \mathcal{R}_{i}\right\rangle$.

$\left(\mathbf{Q}_{2}\right) \quad \mu_{n}=o(1), \mu_{n} \leq \alpha$, and $\mu_{n} \rho_{n}>K \varepsilon_{n}$ for any $n \geq 1$.

$\left(\mathbf{Q}_{3}\right) \quad \varepsilon_{n+1}>8 \max \left\{|R|, R \in \mathcal{R}_{n}\right\}=O\left(\rho_{n}\right)$.

The following lemma shows that if $\alpha$ is small enough and $K$ is big enough, $\mathcal{Q}(\alpha, K)-$ presentations have properties resembling the properties of ordinary small cancellation presentations.

Lemma 4.13 Let (18) be a $\mathcal{Q}\left(.01,10^{6}\right)$-presentation. Then the following conditions hold.

(a) For every $n \geq 1$, Lemma 4.6 applies to $H=G_{n-1}$ and $H_{1}=G_{n}=\left\langle H \mid \mathcal{R}_{n}\right\rangle$. In particular, $G_{n}$ is $\delta_{n}$-hyperbolic, where $\delta_{n} \leq 4 \max _{R \in \mathcal{R}_{n}}|R|$.

(b) $\varepsilon_{n}=o\left(\rho_{n}\right)$.

(c) $\rho_{n}=o\left(\rho_{n+1}\right)$; in particular, $\rho_{n} \rightarrow \infty$ as $n \rightarrow \infty$ and $\delta_{n}=o\left(\rho_{n+1}\right)$.

(d) $\rho_{n}=o\left(r_{S}\left(G_{n} \rightarrow G_{n+1}\right)\right)$, where $r_{S}$ is the injectivity radius.

Proof The first assertion easily follows from $\left(\mathbf{Q}_{0}\right)-\left(\mathbf{Q}_{2}\right)$ by induction. Assertions (b) and (c) follow immediately from $\left(\mathbf{Q}_{2}\right)$ and $\left(\mathbf{Q}_{3}\right)$. Finally given $g \in \operatorname{Ker}\left(G_{n} \rightarrow G_{n+1}\right)$, $g \neq 1$, we consider a geodesic (in $G_{n}$ ) word $W$ representing $g$ and a minimal van Kampen diagram over $G_{n+1}$ with boundary label $W$. Applying Lemma 4.6 for $r=1$ and taking into account that words in $\mathcal{R}_{n+1}$ are geodesic in $G_{n}$, we obtain $|W|>\left(1-23 \mu_{n+1}\right)|\partial \Pi|-2 \varepsilon_{n}$, where $\Pi$ is an $\mathcal{R}_{n+1}$-cell provided by Lemma 4.6. Hence $|g| \geq(1-o(1)) \rho_{n+1}$. Combining this with (c) we obtain (d). 
Definition 4.14 From now on the condition $\mathcal{Q}=\mathcal{Q}\left(.01,10^{6}\right)$ will be called the graded small cancellation condition.

The following statement is an immediate corollary of Lemma 4.7. It shows, in particular, that the class of groups admitting graded small cancellation presentations is large.

Corollary 4.15 Let $R_{1}, R_{2}, \ldots$ be an infinite set of distinct cyclically reduced words in a finite alphabet $S, \mathcal{R}_{i}$ the set of all cyclic shifts of $R_{i}^{ \pm 1}$, and $\mathcal{R}=\bigcup_{i=1}^{\infty} \mathcal{R}_{i}$. Assume that conditions $\left(S C_{1}\right)$ for $\lambda=1 / 100$ and $\left(S C_{2}\right)$ of Lemma 4.7 are satisfied. Then there is an infinite sequence $i_{1}<i_{2}<\cdots$ such that $\left\langle S \mid \bigcup_{j=1}^{\infty} \mathcal{R}_{i_{j}}\right\rangle$ satisfies the graded small cancellation condition.

Definition 4.16 Given an ultrafilter $\omega$ and a scaling sequence $d=\left(d_{n}\right)$, we say that a sequence of real numbers $f=\left(f_{n}\right)$ is $(\omega, d)$-visible if there exists a subsequence $\left(f_{n_{i}}\right)$ of $f$ such that $f_{n_{i}}=\Theta_{\omega}\left(d_{i}\right)$.

Theorem 4.17 For any group $G$ having a graded small cancellation presentation, any ultrafilter $\omega$, and any sequence of scaling constants $d=\left(d_{n}\right)$, the asymptotic cone $\operatorname{Con}^{\omega}(G, d)$ is a circle-tree. $\operatorname{Con}^{\omega}(G, d)$ is an $\mathbb{R}$-tree if and only if the sequence $\left(\rho_{n}\right)$ from Definition 4.12 is not $(\omega, d)$-visible.

The proof of the theorem is divided into a sequence of lemmas. Throughout the rest of the section we fix arbitrary scaling sequence $d=\left(d_{n}\right)$ and ultrafilter $\omega$.

Let us fix a group $G$ having a graded small cancellation presentation (18). In what follows, we denote by dist $_{S}$ (respectively dist ${ }_{\text {Hau }}$ ) the distance (respectively the Hausdorff distance) in the Cayley graph $\Gamma(G, S)$.

Lemma 4.18 Any subword of any word $R_{n} \in \mathcal{R}_{n}$ of length at most $\left|R_{n}\right| / 2$ is $\left(1-o_{\omega}(1), 0\right)$-quasigeodesic.

Proof Suppose $U_{n}=V_{n}$ in $G$, where $U_{n}$ is a subword of $R_{n} \in \mathcal{R}_{n},\left|U_{n}\right| \leq\left|R_{n}\right| / 2$, and $V_{n}$ is a geodesic word in $G$. By Lemma 4.13, $U_{n}=V_{n}$ in $G_{n} \omega$-almost surely. Let $\Delta_{n}$ be a minimal diagram over $G_{n}$ with boundary $p_{n} q_{n}$, where $\operatorname{Lab}\left(p_{n}\right)=U_{n}$, $\operatorname{Lab}\left(q_{n}\right)=V_{n}^{-1}$. If $\Delta_{n}$ has no $\mathcal{R}_{n}$-cells, then $\left|U_{n}\right|=\left|V_{n}\right|$ since $R_{n}$ is geodesic in $G_{n-1}$. Thus we may assume that $\Delta_{n}$ has at least one $\mathcal{R}_{n}$-cell. Let $\Pi_{n}$ be the $\mathcal{R}_{n}$-cell, $\Gamma_{n}^{1}, \Gamma_{n}^{2}$ the contiguity diagrams to $p, q$, respectively, provided by Lemma 4.6.

Observe that $\lim ^{\omega}\left(\Pi_{n}, \Gamma_{n}^{2}, q_{n}\right) \leq 1 / 2$. (Otherwise $\left(\Pi_{n}, \Gamma_{n}^{2}, q_{n}\right)>1 / 2+2 \varepsilon_{n} /\left|\Pi_{n}\right|$ $\omega$-almost surely as $2 \varepsilon_{n} /\left|\Pi_{n}\right|=o(1)$ and $V_{n}$ could not be geodesic by Lemma 4.4.) 
Thus $\lim ^{\omega}\left(\Pi_{n}, \Gamma_{n}^{1}, p_{n}\right) \geq 1 / 2>\mu_{n}$. Hence ||$\partial \Pi_{n}|-| R_{n}|| \leq 2 \varepsilon_{n}$ by Lemma 4.5. Now there are two cases to consider.

Case 1 If $\lim ^{\omega}\left(\Pi_{n}, \Gamma_{n}^{2}, q_{n}\right)=1 / 2$, then

$$
\begin{aligned}
\left|V_{n}\right| & \geq\left(1 / 2-o_{\omega}(1)\right)\left|\partial \Pi_{n}\right|-2 \varepsilon_{n} \\
& \geq\left(1 / 2-o_{\omega}(1)\right)\left(\left|R_{n}\right|-2 \varepsilon_{n}\right)-2 \varepsilon_{n} \geq\left(1-o_{\omega}(1)\right)\left|U_{n}\right|
\end{aligned}
$$

and we are done.

Case 2 If $\lim ^{\omega}\left(\Pi_{n}, \Gamma_{n}^{2}, q_{n}\right)<\theta<1 / 2$, then $\lim ^{\omega}\left(\Pi_{n}, \Gamma_{n}^{1}, p_{n}\right)>1-\theta$ and we get a contradiction as

$$
\left|U_{n}\right|>(1-\theta)\left|\partial \Pi_{n}\right|-2 \varepsilon_{n} \geq(1-\theta)\left(\left|R_{n}\right|-2 \varepsilon_{n}\right)-2 \varepsilon_{n}>\frac{1}{2}\left|R_{n}\right|
$$

$\omega$-almost surely.

Definition 4.19 Let $\mathcal{A}$ denote the set of all loops in the Cayley graph $\Gamma(G, S)$ labeled by words from the set of relators $\mathcal{R}$ (see (18)). We say that a sequence $\left(p_{n}\right)$ of elements of $\mathcal{A}$ is asymptotically visible (relative to the scaling sequence $d$ and ultrafilter $\omega$ ) if

$$
\left|p_{n}\right|=\Theta_{\omega}\left(d_{n}\right) \text { and } \operatorname{dist}_{S}\left(1, p_{n}\right)=O_{\omega}\left(d_{n}\right) .
$$

By $\mathcal{C}=\mathcal{C}(d, \omega)$ we denote the collection of all distinct limits $\lim ^{\omega} p_{n}$, where $\left(p_{n}\right)$ ranges in the set of all asymptotically visible sequences of elements of the set $\mathcal{A}$.

Lemma 4.20 Every piece from $C=\lim ^{\omega} p_{n} \in \mathcal{C}$ is isometric to a circle of length $\lim ^{\omega}\left(\left|p_{n}\right| / d_{n}\right)$.

Proof By Lemma 4.18, $p_{n}$ equipped with the metric induced from $\Gamma(G, S)$ is $(1-$ $\left.o_{\omega}(1), 0\right)$-quasi-isometric to a circle of length $\left|p_{n}\right|$ and our lemma follows. Checking details is straightforward and we leave this to the reader.

The next observation is quite trivial and follows immediately from Property $\left(\mathbf{Q}_{3}\right)$ of the graded small cancellation condition (Definition 4.12) and Lemma 4.13 (c).

Lemma 4.21 Suppose that the sequence $\left(\rho_{n}\right)$ is $(\omega, d)$-visible. Let $\left(\mathcal{R}_{i_{n}}\right)$ be a subsequence of $\left(\mathcal{R}_{n}\right)$, such that $\rho_{i_{n}}=\Theta_{\omega}\left(d_{n}\right)$. Then for any asymptotically visible sequence $\left(p_{n}\right)$ where $\operatorname{Lab}\left(p_{n}\right) \in \mathcal{R}$, we have $\operatorname{Lab}\left(p_{n}\right) \in \mathcal{R}_{i_{n}} \omega$-almost surely.

Lemma 4.22 Suppose that for some asymptotically visible sequences $\left(p_{n}\right)$ and $\left(q_{n}\right)$, the intersection $\lim ^{\omega} p_{n} \cap \lim ^{\omega} q_{n}$ contains at least two distinct points. Then $\lim ^{\omega} p_{n}=$ $\lim ^{\omega} q_{n}$. 
Proof The assumptions of the lemma imply that for every $n \in \mathbb{N}$, there exist quadrangles $Q_{n}=p_{n}^{\prime} s_{n} q_{n}^{\prime} t_{n}$ in $\Gamma(G, S)$ such that $p_{n}^{\prime}$ and $q_{n}^{\prime}$ are subpaths of $p_{n}$ and $q_{n}$, respectively, and $s_{n}, t_{n}$ are geodesics such that

$$
\begin{aligned}
\left|p_{n}\right| & =\Theta_{\omega}\left(d_{n}\right), & \left|q_{n}\right| & =\Theta_{\omega}\left(d_{n}\right), \\
\left|s_{n}\right| & =o_{\omega}\left(d_{n}\right), & \left|t_{n}\right| & =o_{\omega}\left(d_{n}\right) .
\end{aligned}
$$

Without loss of generality we may also assume that

$$
\left|p_{n}^{\prime}\right| \leq \frac{1}{2}\left|p_{n}\right|, \quad\left|q_{n}^{\prime}\right| \leq \frac{1}{2}\left|q_{n}\right| .
$$

Let $\left(\mathcal{R}_{i_{n}}\right)$ be the subsequence of $\left(\mathcal{R}_{n}\right)$ provided by Lemma 4.21. Thus $\operatorname{Lab}\left(p_{n}\right)$, $\operatorname{Lab}\left(q_{n}\right) \in \mathcal{R}_{i_{n}} \omega$-almost surely. Note that (20) and (21) imply

$$
\left|Q_{n}\right|=\Theta_{\omega}\left(d_{n}\right)=\Theta_{\omega}\left(\rho_{i_{n}}\right) .
$$

Therefore, by Lemma 4.13 (d) there is a van Kampen diagram $\Xi_{n}$ with the boundary label $\operatorname{Lab}\left(Q_{n}\right)$ having no $\mathcal{R}_{j}$-cells for $j>i_{n} \omega$-almost surely. For simplicity we keep the notation $p_{n}^{\prime}, s_{n}, q_{n}^{\prime}, t_{n}$ for the corresponding parts of $\partial \Xi_{n}$. Let also $\Delta_{n}$ be the diagram obtained from $\Xi_{n}$ by attaching two $\mathcal{R}_{i_{n}}-$ cells $\Sigma_{n}^{1}$ and $\Sigma_{n}^{2}$ along $p_{n}^{\prime}$ and $q_{n}^{\prime}$ respectively so that the natural map $\operatorname{Sk}^{(1)}\left(\Delta_{n}\right) \rightarrow \Gamma(G, S)$ sending parts of $\partial \Xi_{n}$ to the corresponding sides of $Q_{n}$, maps $\partial \Sigma_{n}^{1}$ to $p_{n}$ and $\partial \Sigma_{n}^{2}$ to $q_{n}$. There are two cases to consider.

Case $1 \Xi_{n}$ has no $\mathcal{R}_{i_{n}}$-cells $\omega$-almost surely and hence $\operatorname{Lab}\left(Q_{n}\right)=1$ in $G_{i_{n}-1}$ $\omega$-almost surely. We recall that $\Gamma\left(G_{i_{n}-1}, S\right)$ is $\delta_{i_{n}-1}$-hyperbolic. Let $p_{n}^{\prime \prime}$ be the subpath of $p_{n}^{\prime}$ such that $\operatorname{dist}\left(\left(p_{n}^{\prime \prime}\right)_{ \pm},\left(p_{n}^{\prime}\right)_{ \pm}\right)=\max \left\{\left|s_{n}\right|,\left|t_{n}\right|\right\}+2 \delta_{i_{n}-1}$. (Note that $\left|p_{n}^{\prime}\right|>2\left(\max \left\{\left|s_{n}\right|,\left|t_{n}\right|\right\}+2 \delta_{i_{n}-1}\right) \omega$-almost surely.) By Lemma $2.9, \operatorname{dist}_{\text {Hau }}\left(p_{n}^{\prime \prime}, q_{n}^{\prime}\right) \leq$ $2 \delta_{i_{n}-1}$. Thus we may assume that $\Delta_{n}$ contains a $2 \delta_{i_{n}-1}$-contiguity subdiagram $\Gamma_{n}$ of $\Sigma_{n}^{1}$ to $\Sigma_{n}^{2}$ such that

$$
\left(\Sigma_{n}^{1}, \Gamma_{n}, \Sigma_{n}^{2}\right)=\frac{\left|p_{n}^{\prime}\right|-2\left(\max \left\{\left|s_{n}\right|,\left|t_{n}\right|\right\}+2 \delta_{i_{n}-1}\right)}{\left|\partial \Sigma_{n}^{1}\right|}=\frac{\Theta_{\omega}\left(d_{n}\right)-o_{\omega}\left(d_{n}\right)}{\Theta_{\omega}\left(d_{n}\right)}>\mu_{i_{n}}
$$

$\omega$-almost surely (see Figure 5). Now Lemma 4.5 and Lemma 4.13 imply that

$$
\operatorname{dist}_{\text {Hau }}\left(p_{n}, q_{n}\right) \leq \varepsilon_{n}+2 \delta_{i_{n}-1}=o_{\omega}\left(d_{n}\right) .
$$

Therefore $\lim ^{\omega} p_{n}=\lim ^{\omega} q_{n}$.

Case 2 Suppose now that $\Delta_{n}$ has at least one $\mathcal{R}_{i_{n}}$-cell $\omega$-almost surely. Then by Lemma 4.6, we may assume that there is an $\mathcal{R}_{i_{n}}$-cell $\Pi_{n}$ in $\Xi_{n}$ and disjoint $\varepsilon_{i_{n}}$-contiguity subdiagrams $\Gamma_{n}^{1}, \ldots, \Gamma_{n}^{4}$ of $\Pi_{n}$ to $p_{n}^{\prime}, s_{n}, q_{n}^{\prime}, t_{n}$, respectively, such that

$$
\left(\Pi_{n}, \Gamma_{n}^{1}, p_{n}^{\prime}\right)+\left(\Pi_{n}, \Gamma_{n}^{2}, s_{n}\right)+\left(\Pi_{n}, \Gamma_{n}^{3}, q_{n}^{\prime}\right)+\left(\Pi_{n}, \Gamma_{n}^{4}, t_{n}\right)>1-23 \mu_{i_{n}}
$$



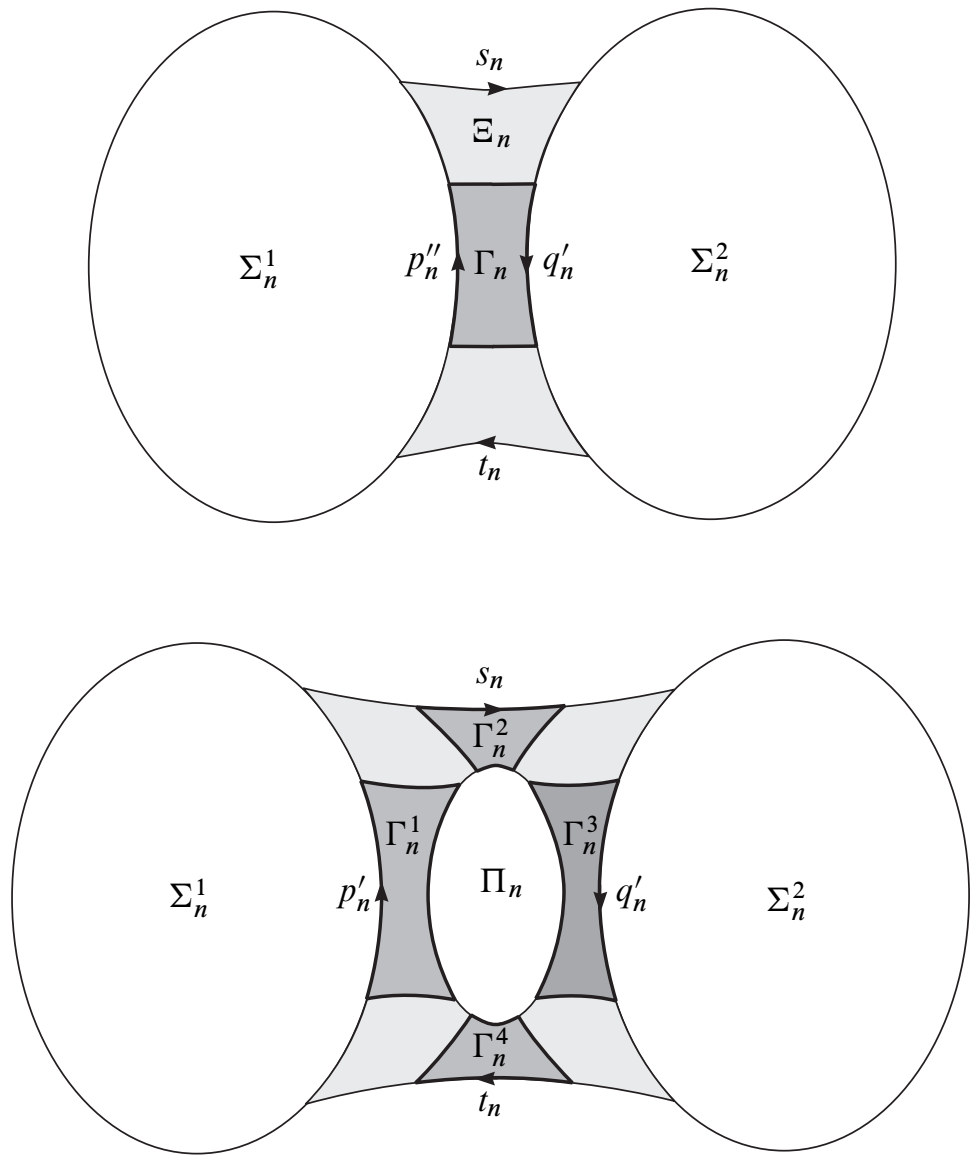

Figure 5: Two cases in the proof of Lemma 4.22

$\omega$-almost surely (Figure 5). Using (21) and Lemma 4.13 one can easily show that

$$
\left(\Pi_{n}, \Gamma_{n}^{2}, s_{n}\right)+\left(\Pi_{n}, \Gamma_{n}^{4}, t_{n}\right)=o_{\omega}(1) .
$$

Hence

$$
\left(\Pi_{n}, \Gamma_{n}^{1}, p_{n}^{\prime}\right)+\left(\Pi_{n}, \Gamma_{n}^{3}, q_{n}^{\prime}\right)=1-o_{\omega}(1) \text {. }
$$

Note that $\Gamma_{n}^{3}$ may also be considered as a contiguity subdiagram of $\Pi_{n}$ to $\Sigma_{n}^{2}$ in $\Delta_{n}$. Suppose first that we have $\left(\Pi_{n}, \Gamma_{n}^{1}, p_{n}^{\prime}\right)=\left(\Pi_{n}, \Gamma_{n}^{1}, \Sigma_{n}^{1}\right) \leq \mu_{i_{n}} \omega$-almost surely. Then $\left(\Pi_{n}, \Gamma_{n}^{3}, \Sigma_{n}^{2}\right)=1-o_{\omega}(1)$. Applying Lemma 4.5 we obtain

$$
|| \partial \Pi_{n}|-| \partial \Sigma_{n}^{2} \|<2 \varepsilon_{n} .
$$


Hence the length of the contiguity arc $v_{n}$ of $\Gamma_{n}^{3}$ to $\Sigma_{n}^{2}$ satisfies

$$
\left|v_{n}\right| \geq\left(1-o_{\omega}(1)\right)\left|\partial \Pi_{n}\right|-2 \varepsilon_{n}=\left(1-o_{\omega}(1)\right)\left|\partial \Sigma_{n}^{2}\right| .
$$

However this contradicts (22).

Therefore $\left(\Pi_{n}, \Gamma_{n}^{1}, p_{n}^{\prime}\right)>\mu_{i_{n}}$ and similarly $\left(\Pi_{n}, \Gamma_{n}^{3}, q_{n}^{\prime}\right)>\mu_{i_{n}}$. Let $w_{n}$ be the image of $\partial \Pi_{n}$ under the natural map $\operatorname{Sk}^{(1)}\left(\Delta_{n}\right) \rightarrow \Gamma(G, S)$ sending $\partial \Sigma_{n}^{1}$ and $\partial \Sigma_{n}^{2}$ to $p_{n}$ and $q_{n}$ respectively. Then using Lemma 4.5 and Lemma 4.13 we obtain

$$
\operatorname{dist}_{\mathrm{Hau}}\left(p_{n}, q_{n}\right) \leq \operatorname{dist}_{\mathrm{Hau}}\left(p_{n}, w_{n}\right)+\operatorname{dist}_{\mathrm{Hau}}\left(q_{n}, w_{n}\right)=o_{\omega}\left(d_{n}\right) .
$$

Thus $\lim ^{\omega}\left(p_{n}\right)=\lim ^{\omega}\left(q_{n}\right)$ again.

Lemma 4.23 Every simple triangle in $\operatorname{Con}^{\omega}(G, d)$ whose sides are limit geodesics is contained in a subset from $\mathcal{C}$.

Proof Suppose that $p q s$ is a simple triangle in $\operatorname{Con}^{\omega}(G, d)$ whose sides are limit geodesics. As in the proof of Theorem 3.3, pqs $=\lim ^{\omega} H_{n}$, where $H_{n}$ is a geodesic hexagon in $\Gamma(G, S)$. Let $\Delta_{n}$ be a van Kampen diagram over (18) with boundary label $\operatorname{Lab}\left(H_{n}\right)$.

First of all we assume that $\left(\rho_{n}\right)$ is $(\omega, d)$-visible and denote by $\left(\mathcal{R}_{i_{n}}\right)$ be the subsequence of $\left(\mathcal{R}_{n}\right)$ provided by Lemma 4.21. Note that $\left|H_{n}\right|=\Theta_{\omega}\left(d_{i_{n}}\right)$. Arguing as in the proof of the previous lemma, one can show that $\Delta_{n}$ has no $\mathcal{R}_{j}$-cells for $j>i_{n}$ $\omega$-almost surely. To simplify our notation we identify the 1 -skeleton of $\Delta_{n}$ with its natural image in $\Gamma(G, S)$.

If $\Delta_{n}$ has no $\mathcal{R}_{i_{n}}$-cells $\omega$-almost surely, we obtain a contradiction as in the third paragraph of the proof of Theorem 3.3. Thus we may assume that $\Delta_{n}$ has at least one $\mathcal{R}_{i_{n}}$-cell $\omega$-almost surely and $\Delta_{n}$ is minimal over $G_{i_{n}}$. By Lemma 4.6 there is an $\mathcal{R}_{i_{n}}$-cell $\Pi_{n}$ in $\Delta_{n}$ and $\varepsilon_{i_{n}}$-contiguity subdiagrams $\Gamma_{n}^{1}, \ldots, \Gamma_{n}^{6}$ of $\Pi_{n}$ to the sides of $H_{n}$ such that $\partial \Pi_{n}$ belongs to the closed $\left(23 \mu_{i_{n}}\left|\partial \Pi_{n}\right|\right)$-neighborhood of the union $\bigcup_{i=1}^{6} \Gamma_{n}^{i}$ in $\Delta_{n} \omega$-almost surely. Let $w_{n}$ denote the natural image of $\partial \Pi_{n}$ in $\Gamma(G, S)$. By Lemma $2.9 w_{n}$ belongs to the closed $\left(23 \mu_{i_{n}}\left|\partial \Pi_{n}\right|+\varepsilon_{i_{n}}+2 \delta_{i_{n}-1}\right)$-neighborhood of $H_{n}$ in $\Gamma(G, S)$. Further Lemma 4.13 implies that $23 \mu_{i_{n}}\left|\partial \Pi_{n}\right|+\varepsilon_{i_{n}}+2 \delta_{i_{n}-1}=o_{\omega}\left(d_{n}\right)$. Hence $\lim ^{\omega} w_{n} \subseteq p q s$. Since $\lim ^{\omega} w_{n}$ is a circle by Lemma 4.20 and $p q s$ is simple, we have $p q s=\lim ^{\omega} w_{n} \in \mathcal{C}$.

Finally we assume that $\left(\rho_{n}\right)$ is not $(\omega, d)$-visible. Let $j_{n}$ be the maximal number such that $\Delta_{n}$ has at least one $\mathcal{R}_{j_{n}}$-cell. Let $\Pi_{n}$ and $\Gamma_{n}^{1}, \ldots, \Gamma_{n}^{6}$ be the $\mathcal{R}_{j_{n}}$-cell and the $\varepsilon_{j_{n}}$-contiguity subdiagrams of $\Pi_{n}$ to sides of $H_{n}$ provided by Lemma 4.6. Again we can easily show that the total length of the contiguity arcs of $\Gamma_{n}^{1}, \ldots, \Gamma_{n}^{6}$ to the sides 
of $H_{n}$ is $\Theta_{\omega}\left(\rho_{j_{n}}\right)$. Since $\left(\rho_{n}\right)$ is not $(\omega, d)$-visible, $\rho_{j_{n}}=\Theta_{\omega}\left(d_{n}\right)$ is impossible. Therefore $\rho_{j_{n}}=o_{\omega}\left(d_{n}\right)$. Hence $\delta_{j_{n}}=o_{\omega}\left(d_{n}\right)$ by Lemma 4.13. This again leads to a contradiction as in the third paragraph of the proof of Theorem 3.3. Thus $\operatorname{Con}^{\omega}(G, d)$ has no nontrivial simple triangles whose sides are limit geodesics, ie, it is tree-graded with respect to the empty collection of pieces (ie, it is an $\mathbb{R}$-tree) by Lemma 2.7 . $\square$

Proof of Theorem 4.17 Let $G$ have a graded small cancellation presentation (18). For any fixed scaling sequence $d=\left(d_{n}\right)$ and any ultrafilter $\omega$, let $\mathcal{C}=\mathcal{C}(d, \omega)$ be the collection of subsets of $\operatorname{Con}^{\omega}(G, d)$ described in Definition 4.19. If $\left(\rho_{n}\right)$ is $(\omega, d)-$ visible, all elements of $\mathcal{C}$ are circles whose radii are uniformly bounded from below and from above by positive constants by Lemma 4.20 and Lemma 4.21. Further by Lemma 4.22, $\mathcal{C}$ satisfies $\left(T_{1}\right)$ (see Definition 2.1). Applying now Lemma 4.23 and Lemma 2.7 we conclude that $\operatorname{Con}^{\omega}(G, d)$ is tree-graded with respect to $\mathcal{C}$, ie, is a circle-tree. If $\left(\rho_{n}\right)$ is not $(\omega, d)$-visible, the same arguments show that $\operatorname{Con}^{\omega}(G, d)$ is tree-graded with respect to $\mathcal{C}$. But $\mathcal{C}$ is empty in this case, hence $\operatorname{Con}^{\omega}(G, d)$ is an $\mathbb{R}$-tree.

\subsection{Groups without free subgroups}

Recall that any torsion-free nonelementary hyperbolic group has an infinite quotient group with finite (or cyclic) proper subgroups [42]. We show in this section that some of these groups have graded small cancellation presentations. In what follows, $H$ denotes a hyperbolic group generated by a finite set $S$.

The following Lemma 4.24 is an analog of Lemma 4.6 for such presentations (and a particular case of [42, Lemma 6.6]), while Lemma 4.25 is a quasigeodesic analog of Lemma 4.4.

Lemma 4.24 For any hyperbolic group $H$ and any $\lambda>0$, there is $\mu_{0}>0$ such that for any $\mu \in\left(0, \mu_{0}\right]$ and any $c \geq 0$, there are $\varepsilon \geq 0$ and $\rho>0$ with the following property:

Suppose a finite symmetrized presentation $H_{1}=\langle H \mid \mathcal{R}\rangle$ satisfies the condition $C(\varepsilon, \mu, \lambda, c, \rho)$, and $\Delta$ is a minimal diagram over $H_{1}$ whose boundary is a product of $(\lambda, c)$-quasigeodesic paths $p$ and $q$. Then provided $\Delta$ has an $\mathcal{R}$-cell, there exists an $\mathcal{R}$-cell $\Pi$ in $\Delta$ and disjoint $\varepsilon$-contiguity subdiagrams $\Gamma_{1}$ and $\Gamma_{2}$ (one of them may be absent) of $\Pi$ to $p$ and $q$, respectively, such that $\left(\Pi, \Gamma_{1}, p\right)+\left(\Pi, \Gamma_{2}, q\right)>1-23 \mu$.

Lemma 4.25 Let a presentation $H_{1}=\langle H \mid \mathcal{R}\rangle$ satisfy a $C(\varepsilon, \mu, \lambda, c, \rho)$-condition with $\mu<\lambda^{2} / 100$ and $\rho>2 \mu^{-1}(c+2 \varepsilon)$. Let $\Delta$ be a minimal diagram over $H_{1}$ with a $(\lambda, c)$-quasigeodesic subpath $q$ of the boundary, and $\Gamma$ a contiguity subdiagram of an $\mathcal{R}$-cell $\Pi$ to $q$. Then $\psi=(\Pi, \Gamma, q)<1-24 \mu$. 
Proof Let $p_{1} q_{1} p_{2} q_{2}$ be the boundary of the contiguity subdiagram of $\Pi$ to $q$, where $q_{1} p=\partial \Pi$ and $q_{2}$ is a subpath of $q$. Since $q_{2}$ is a $(\lambda, c)$-quasigeodesic subpath of $q$, we have $\lambda\left|q_{2}\right|-c \leq\left|p_{2}\right|+|p|+\left|p_{1}\right| \leq 2 \varepsilon+(1-\psi)|\partial \Pi|$. On the other hand, $\left|q_{2}\right| \geq \lambda\left|q_{1}\right|-c-\left|p_{1}\right|-\left|p_{2}\right|$ since the path $q_{1}$ is $(\lambda, c)$-quasigeodesic. Hence $\left|q_{2}\right| \geq \lambda \psi|\partial \Pi|-c-2 \varepsilon$. These two estimates for $\left|q_{2}\right|$ give us the inequality

$$
\lambda^{-1}(|\partial \Pi|(1-\psi)+2 \varepsilon+c) \geq \psi \lambda|\partial \Pi|-c-2 \varepsilon,
$$

that is,

$$
\psi \leq\left(\lambda^{-1}|\partial \Pi|+\left(1+\lambda^{-1}\right)(c+2 \varepsilon)\right)\left(|\partial \Pi|\left(\lambda^{-1}+\lambda\right)\right)^{-1} .
$$

Since $|\partial \Pi| \geq \rho$, we obtain from the assumptions of the lemma

$$
\psi \leq \frac{\lambda^{-1} \rho+\left(1+\lambda^{-1}\right)(c+2 \varepsilon)}{\rho\left(\lambda^{-1}+\lambda\right)}<\frac{1+\mu}{1+100 \mu}<1-24 \mu .
$$

Below we say that a bi-infinite path $p$ in the Cayley graph of a group generated by a finite set $S$ is $V$-periodic (or just periodic), if $p$ is labeled by the bi-infinite power of some word $V$ in $S^{ \pm 1}$.

Theorem 4.26 (1) Let $G$ be an arbitrary nonelementary hyperbolic group. Then there exists an infinite torsion quotient group $Q_{1}$ of $G$ admitting a graded small cancellation presentation.

(2) Let $G$ be an arbitrary torsion free noncyclic hyperbolic group with a finite set of generators $S$. Then there exists an infinite non-Abelian torsion free quotient group $Q_{2}$ of $G$ admitting a graded small cancellation presentation and such that all proper subgroups of $Q_{2}$ are cyclic. Moreover, every periodic bi-infinite path in the Cayley graph $\Gamma\left(Q_{2}, S\right)$ is a Morse quasigeodesic.

Proof (1) Infinite torsion quotient group $Q_{1}$ of an arbitrary nonelementary hyperbolic group $G$ was constructed in [42, Corollary 2] as a direct limit of a sequence of hyperbolic groups $G=G(0) \rightarrow G(1) \rightarrow \cdots \rightarrow G(i-1) \rightarrow G(i) \rightarrow \cdots$, where each step is a transition from $H=G(i-1)$ to $H_{1}=G(i)=\left\langle G(i-1) \mid V^{m}\right\rangle$ for a word $V=V_{i}$ and a sufficiently large $m=m_{i}$. Lemmas 4.1 and 6.7 of [42] claim that the set of the cyclic shifts of the words $V^{ \pm 1}$ satisfy a $C(\varepsilon, \mu, \lambda, c, \rho)$-condition, where $\lambda=\lambda_{i}=\lambda(V, i-1)>0, c=c_{i}=c(V, i-1) \geq 0$, the positive $\mu=\mu_{i}$ can be selected arbitrary small, then $\varepsilon=\varepsilon_{i}$ can be chosen arbitrary large, and afterwards $m$, and therefore $\rho=\rho_{i}$, can be chosen arbitrary large.

It follows from Lemma 4.11 that one can replace the defining word $V_{i}^{m}$ by a conjugate in $H$ word $R_{i}$ having minimal length in its conjugacy class, so that the set of cyclic shifts of $R_{i}$ satisfy $C\left(\varepsilon_{i}^{\prime}, \mu_{i}^{\prime}, \rho_{i}^{\prime}\right)$-condition with parameters $\varepsilon_{n}^{\prime}, \mu_{n}^{\prime}, \rho_{n}^{\prime}(n=1,2, \ldots)$ satisfying the Definition 4.12. This proves the first statement of the proposition. 
(2) To construct $Q_{2}$ we denote by $\mathcal{F}$ the set of all 2-generated subgroups of $G$ and enumerate all elements $p_{1}, p_{2}, \ldots$ of the set $\mathcal{P}=S \times \mathcal{F}$. We set $G(0)=G$ and proceed by induction. Suppose that a (torsion-free) hyperbolic group $G(i-1)$ and relators $R_{1}, \ldots, R_{i-1}$ are already constructed. Then we consider the first pair, say $p_{k}=(s, K) \in \mathcal{P}$, such that the image $K^{\prime}$ of $K$ in $G(i-1)$ is nonelementary and the image $s^{\prime}$ of $s$ in $G(i-1)$ does not belong to $K^{\prime}$. As in the proof of [42, Corollary 1], we can choose a word $R_{i}$ of the form $R_{i} \equiv X_{0} U^{m} X_{1} U^{m} \cdots X_{l} U^{m}$, where $X_{0}$ represents an element of $s K$ and $U, X_{1}, \ldots, X_{l}$ represent elements of $K$, such that the set of all cyclic shifts of $R_{i}^{ \pm 1}$ satisfies a $C(\varepsilon, \mu, \lambda, c, \rho)$-condition. Here $\lambda=\lambda_{i}>0$, the positive $\mu=\mu_{i}$ can be selected arbitrary small, and then $c=c_{i} \geq 0$ arbitrary large, then $\varepsilon=\varepsilon_{i}$ can be chosen arbitrary large, and afterwards $m$, and therefore $\rho=\rho_{i}$, can be chosen arbitrary large. Such a choice of the parameters is guaranteed by Lemmas 4.2 and 6.7 of [42]. Then $G(i)=\left\langle G(i-1) \mid R_{i}\right\rangle$, and the group $Q_{2}$ is defined to be the limit of the sequence $G=G(0) \rightarrow G(1) \rightarrow \cdots \rightarrow G(i) \rightarrow \ldots$ Hence, as in the first part of the proof, one can choose the parameters so that $Q_{2}$ has a graded small cancellation presentation. As in [42, Corollary 1], $Q_{2}$ is a non-Abelian torsion free group with cyclic proper subgroups. (The only difference is that now we are adding only one relation $R_{i}$ when passing from $G(i-1)$ to $G(i)$, while in [42], the set $\mathcal{F}$ was enumerated, and, for given $K \in \mathcal{F}$, one imposed finitely many relations to obtain $G(i)$, namely, one relation for every $s \in S$.)

To ensure the Morse property for bi-infinite periodic paths in $\Gamma\left(Q_{2}, S\right)$ we have to make the following additional changes in the scheme from [42]. For every $i=1,2, \ldots$, when passing from $G(i-1)$ to $G(i)$ we fix a set of words $\mathcal{V}_{i}$ in $S^{ \pm 1}$ of lengths at most $i$ having infinite order in $G_{i-1}$. Arbitrary power of a word from $\mathcal{V}_{i}$ is $(\lambda(i), c(i))-$ quasigeodesic in the hyperbolic group $G(i-1)$ for some $\lambda(i)>0$ and $c(i) \geq 0$, and one can chose the constants $\lambda_{i}$ and $c_{i}$ in the previous paragraph so that $\lambda_{i} \leq \lambda(i)$ and $c_{i} \geq c(i)$. Then [42, Lemma 2.5] allows us to chose the words $R_{i}$ and the parameters in the previous paragraph so that the following is true.

$(*)$

$\left\{\begin{array}{l}\text { Let } \Delta \text { be a minimal diagram over } G(i), q \text { a part of } \partial \Delta \text { such that } \operatorname{Lab}(q)= \\ V_{i}^{n} \text { for some } 1 \leq i \leq s, n \in \mathbb{N} \text {. Then } \Delta \text { contains no } \varepsilon_{i} \text {-contiguity } \\ \text { subdiagrams of } R_{i} \text {-cells to } q \text { with contiguity degree at least } \mu_{i} .\end{array}\right.$

Assume that $V$ is a word representing a nontrivial element in $Q_{2}$. Then $V \in \mathcal{V}_{i}$ for some $i$. Assume that for some $n>0, V^{n}=U$ in $Q_{2}$, where $U$ is a geodesic word in $Q_{2}$. Let $j=j(n)$ be the smallest positive integer such that $V^{n}=U$ in $G(j)$. Suppose that $j \geq i$ (hence $V \in \mathcal{V}_{j}$ ). Consider a minimal diagram $\Delta$ over $G(j)$ with $\partial \Delta=p q$, where $\operatorname{Lab}(p) \equiv V^{n}$ and $\operatorname{Lab}\left(q^{-1}\right) \equiv U$. Let $\Pi, \Gamma_{1}, \Gamma_{2}$ be the $\mathcal{R}_{j}$-cell of $\Delta$ and the $\varepsilon_{j}$-contiguity diagrams of $\Pi$ to $p$ and $q$, respectively, provided by Lemma 4.24. Then $\left(\Pi, \Gamma_{1}, p\right)<\mu_{j}$ by $(*)$, and so $\left(\Pi, \Gamma_{2}, q\right)>1-24 \mu_{j}$ that contradicts 
Lemma 4.25. Hence $j<i$, ie, $V^{n}=U$ in the hyperbolic group $G(i)$, where $i$ is independent of $n$. This implies that any bi-infinite $V$-periodic path in $\Gamma\left(Q_{2}, S\right)$ is quasigeodesic.

It remains to prove that any bi-infinite $V$-periodic path in $\Gamma\left(Q_{2}, S\right)$ is Morse. Let us fix arbitrary $L, C>0$ and consider any $(L, C)$-quasigeodesic word $W$ such that $V^{n}=W$ in $Q_{2}$ for some $n$. As above let $j$ be the smallest positive integer such that $V^{n}=W$ in $G(j)$.

Since in a hyperbolic group every bi-infinite periodic geodesic is Morse [2], we would finish the proof if we show that $j$ can be bounded from above by some constant $J=J(L, C)$ independent of $n$. Let us choose $J$ so that $\lambda_{J}<L, C<c_{J}$, and $V \in \mathcal{V}_{J}$. Again we consider a minimal diagram $\Delta$ over $Q_{2}$ with $\partial \Delta=p q$, where $\operatorname{Lab}(p) \equiv V^{n}$, and $\operatorname{Lab}\left(q^{-1}\right) \equiv W$.

By contradiction, assume that $\operatorname{rank}(\Delta)=k>J$. Let $\Pi, \Gamma_{1}, \Gamma_{2}$ be the $\mathcal{R}_{k}$-cell of $\Delta$ and the $\varepsilon_{j}$-contiguity diagrams of $\Pi$ to $p$ and $q$, respectively, provided by Lemma 4.24. Then $\left(\Pi, \Gamma_{2}, q\right)>1-24 \mu_{j}$ as above, contrary to Lemma 4.25.

Remark 4.27 In a similar way, one can use methods of [42] to construct an infinite group $Q$ admitting a graded small cancellation presentation, and such that all proper subgroups of $Q$ are finite. In that construction, one would have to use [42, Theorem 4]. Note that there exists a slight error in the formulation of that theorem. Let $E_{0}$ be the elementary group and $C$ its infinite cyclic normal subgroup from the formulation of [42, Theorem 4]. Since the group $H$ satisfies the quasi-identity

$$
x^{2} y=y x^{2} \rightarrow x y=y x,
$$

the center $Z$ of $E_{0}$ has a finite odd index in $E_{0}$ by [42, Proposition 2]. Hence $Z$ contains the Sylow 2-subgroup $P_{2}$ of $E_{0}$ and an infinite cyclic subgroup $C$ such that the product $C P_{2}$ is of odd index in $E_{0}$. To make the formulation of [42, Theorem 4] correct, one needs to add the condition that the cyclic subgroup $C$ in $E_{0}$ is chosen with this additional property, namely, the order of $E_{0} /\left(C P_{2}\right)$ is odd. (This condition was used in the proof of [42, Theorem 4].)

Also the direct limits of hyperbolic groups in [35] can be chosen satisfying Condition (3) of Theorem 3.3. Hence there exist torsion and torsion free examples of divisible (and even verbally complete) lacunary hyperbolic groups.

\subsection{Floyd boundary}

Finally we note a relation between cut points in asymptotic cones and the Floyd boundary. Recall that the Floyd boundary [16] $\partial G$ of a finitely generated group 
$G=\langle S\rangle$ is defined as follows. Let $\operatorname{dist}_{F}$ be the metric on $\Gamma=\Gamma(G, S)$ obtained by setting the lengths of each edge $e$ to be equal to $(1+\operatorname{dist}(e, 1))^{-2}$. It is easy to see that $\Gamma$ is bounded with respect to $\operatorname{dist}_{F}$. Let $\bar{\Gamma}$ be the metric completion of $\left(\Gamma, \operatorname{dist}_{F}\right)$. Then $\partial G=\bar{\Gamma} \backslash \Gamma$.

The Floyd boundary of a group $G$ is a quasi-isometry invariant. If $\partial G$ consists of 0 (respectively 2 ) points, $G$ is finite (respectively virtually cyclic). If $\partial G$ consists of 0,1 or 2 points it is said to be trivial. Otherwise it is uncountable (and, moreover, $\partial G$ is a boundary in the sense of Furstenberg). If $\partial G$ is nontrivial, $G$ contains a free non-Abelian subgroup. In particular, $G$ is trivial for any amenable group. The class of groups with nontrivial Floyd boundary includes nonelementary hyperbolic groups, nonelementary geometrically finite Kleinian groups, groups with infinitely many ends, and many other examples. (For more details we refer to Karlsson [29].)

Proposition 4.28 Let $G$ be a finitely generated group whose Floyd boundary consists of at least 2 points. Then all asymptotic cones of $G$ have cut points.

Proof Let us fix a scaling sequence $d=\left(d_{n}\right)$ and an ultrafilter $\omega$. Let also $\left(x_{n}\right),\left(y_{n}\right)$ be sequences of elements of $G$ that converge to distinct points $x, y \in \partial G$. For each $x_{n}$ we fix a geodesic $\gamma_{n}$ in $\Gamma(G, S)$ connecting $x_{n}$ to 1 . Since $\Gamma(G, S)$ is locally finite, there is an infinite ray $\gamma$ such that $\gamma_{-}=1$ and the combinatorial length of the common part of $\gamma$ and $\gamma_{n}$ tends to $\infty$ as $n \rightarrow \infty$. This means, in particular, that the sequence of vertices of $\gamma$ converge to $x$ as $n \rightarrow \infty$. Thus we may assume that $\left|x_{n}\right|=n$ and $\left|y_{n}\right|=n$. Consider the subsequences $a_{n}=x_{\left[d_{n}\right]}$ and $b_{n}=y_{\left[d_{n}\right]}$ and set $a=\left(a_{n}\right)^{\omega}$, $b=\left(b_{n}\right)^{\omega}$. Note that $\operatorname{dist}\left(a,(1)^{\omega}\right)=\operatorname{dist}\left(b,(1)^{\omega}\right)=1$.

Suppose that $\operatorname{Con}^{\omega}(G, d)$ has no cut points. Then for some $\varepsilon>0$, there is a path $p$ in $\operatorname{Con}^{\omega}(G, d) \backslash$ Ball $\left((1)^{\omega}, \varepsilon\right)$ of some length $L$ connecting $a$ to $b$. Now applying standard methods it is easy to show that $a_{n}$ and $b_{n}$ can be connected by a path $p_{n}$ in $\Gamma(G, S)$ such that $\left|p_{n}\right|<2 L d_{n}$ and $p_{n}$ avoids the ball of radius $\varepsilon d_{n} / 2$ centered at 1 in $\Gamma(G, S)$ (with respect to the combinatorial metric) $\omega$-almost surely. Hence we have

$$
\operatorname{dist}_{F}\left(a_{n}, b_{n}\right) \geq \frac{2 L d_{n}}{\left(\varepsilon d_{n} / 2\right)^{2}}=o(1) .
$$

Thus $\left(a_{n}\right)$ and $\left(b_{n}\right)$ converge to the same points of the Floyd boundary and we get a contradiction.

We note that the converse to Proposition 4.28 does not hold. Indeed all asymptotic cones of groups constructed in this section have cut points by Theorem 4.17. On the other hand their Floyd boundary consists of a single point since they contain no non-Abelian free subgroups and are not virtually cyclic. 
Corollary 4.29 There exists a lacunary hyperbolic group $G$ such that the Floyd boundary $\partial G$ consists of a single point and all asymptotic cones of $G$ are circle-trees.

\section{Central extensions of lacunary hyperbolic groups}

\subsection{Asymptotic cones of group extensions}

In this section, we obtain some results about asymptotic cones of group extensions. These results are used in the next two sections.

Lemma 5.1 (Asymptotic cones of isometry groups) Suppose that a finitely generated group $G$ acts isometrically on a metric space $X$. Fix an arbitrary point $x \in X$. Then the map $\alpha: G \rightarrow X$ defined by $\alpha(g)=g x$ for any $g \in G$ induces a continuous map $\hat{\alpha}: \operatorname{Con}^{\omega}(G, d) \rightarrow \operatorname{Con}^{\omega}(X, d)$.

Proof Note that the map $\alpha$ is C-Lipschitz for

$$
C=\max \left\{\operatorname{dist}(x, s x) \mid s \in S^{ \pm 1}\right\},
$$

where $S$ is a finite generating set of $G$. Indeed if $g=s_{1} \cdots s_{n}$ for some $g \in G$ and $s_{1}, \ldots, s_{n} \in S^{ \pm 1}$, then

$$
\operatorname{dist}(x, g x) \leq \sum_{i=1}^{n} \operatorname{dist}\left(g_{i-1} x, g_{i-1} s_{i} x\right) \leq \sum_{i=1}^{n} \operatorname{dist}\left(x, s_{i} x\right) \leq C n \text {. }
$$

where $g_{0}=1$ and $g_{i}=s_{1} \cdots s_{i}$ for $1 \leq i \leq n$. Thus for any scaling sequence $d=\left(d_{n}\right)$ and any nonprincipal ultrafilter $\omega$, the map $\left(g_{n}\right)^{\omega} \mapsto\left(g_{n} x\right)^{\omega}$ from $\operatorname{Con}^{\omega}(G, d)$ to $\operatorname{Con}^{\omega}(X, d)$ is continuous.

Given a group $G$ generated by a finite set $S$ and a normal subgroup $N$ of $G$, we endow the group $G$ and the quotient group $G / N$ by the word metric with respect to the set $S$ and its image in $G / N$, respectively. We also assume that $N$ is endowed with the metric induced from $G$. Thus for any $d$ and $\omega, \operatorname{Con}^{\omega}(N, d)$ may be considered as a subset of $\operatorname{Con}^{\omega}(G, d)$. Set $x=1 \in G / N$. Then Lemma 5.1 applied to the natural action of $G$ on the quotient group $G / N$ by left multiplications gives us the map $\widehat{\alpha}$ : $\operatorname{Con}^{\omega}(G, d) \rightarrow$ $\operatorname{Con}^{\omega}(G / N, d)$. Note that $\alpha$ is the natural homomorphism $G \rightarrow G / N$ in this case. Given $b \in \operatorname{Con}^{\omega}(G / N, d)$, we call the subset $\mathcal{F}_{b}=\hat{\alpha}^{-1}(b) \subseteq \operatorname{Con}^{\omega}(G, d)$ fiber.

Recall that the group

$$
G_{e}^{\omega}(d)=\left\{\left(g_{n}\right) \in \prod^{\omega} G|| g_{n} \mid=O_{\omega}\left(d_{n}\right)\right\}
$$


acts transitively by isometries on $\operatorname{Con}^{\omega}(G, d)$ by bigg multiplication. Let $N_{e}^{\omega}(d)$ be the subgroup of $G_{e}^{\omega}(d)$ defined as follows:

$$
N_{e}^{\omega}(d)=\left\{\left(g_{n}\right) \in \prod^{\omega} N|| g_{n} \mid=O_{\omega}\left(d_{n}\right)\right\} .
$$

Theorem 5.2 (Asymptotic cones of quotient groups) Let $G=\langle S\rangle$ be a finitely generated group $G, N$ a normal subgroup of $G$ endowed with the metric induced from $G$. Then the following conditions hold.

(a) The map $\hat{\alpha}$ is surjective.

(b) For any $b \in \operatorname{Con}^{\omega}(G / N, d)$, we have $\mathcal{F}_{b}=\gamma \operatorname{Con}^{\omega}(N, d)$ for some element $\gamma \in G_{e}^{\omega}(d)$.

(c) The action of $G_{e}^{\omega}(d)$ permutes fibers, that is, for any $b \in \operatorname{Con}^{\omega}(G / N, d)$ and $\gamma \in G_{e}^{\omega}(d), \gamma \mathcal{F}_{b}$ is a fibre.

(d) The action of $N_{e}^{\omega}(d)$ stabilizes each fiber (as a set) and acts on each fiber transitively.

(e) If $\operatorname{Con}^{\omega}(N, d)$ is discrete, the map $\hat{\alpha}: \operatorname{Con}^{\omega}(G, d) \rightarrow \operatorname{Con}^{\omega}(G / N, d)$ is locally isometric. If $\operatorname{Con}^{\omega}(N, d)$ consists of a single point, then $\operatorname{Con}^{\omega}(G, d)$ and $\operatorname{Con}^{\omega}(G / N, d)$ are isometric.

Proof Let $\sigma: G / N \rightarrow G$ be a section that assigns to each element $x \in G / N$ a shortest preimage of $x$ in $G$. If $h=\left(h_{n} N\right)^{\omega}$ is a point in $\operatorname{Con}^{\omega}(G / N, d)$, then $\left.g=\left(\sigma\left(h_{n} N\right)\right)^{\omega}\right)$ belongs to $\operatorname{Con}^{\omega}(G, d)$ and $\hat{\alpha}(g)=h$. Thus $\hat{\alpha}$ is surjective.

Further for any $b \in \operatorname{Con}^{\omega}(G / N, d)$, we have

$$
\mathcal{F}_{b}=\left\{\left(g_{n}\right)^{\omega} \in \operatorname{Con}^{\omega}(G, d) \mid\left(\alpha\left(g_{n}\right)\right)^{\omega}=b\right\} .
$$

Let us fix any element $\left(f_{n}\right)^{\omega} \in \mathcal{F}_{b}$ and set $\gamma=\left(f_{n}\right)$. Clearly $\gamma \operatorname{Con}^{\omega}(N, d) \subseteq \mathcal{F}_{b}$. If $g=\left(g_{n}\right)^{\omega} \in \mathcal{F}_{b}$, then $\left(\alpha\left(f_{n}\right)\right)^{\omega}=\left(\alpha\left(g_{n}\right)\right)^{\omega}$, ie, $\left|\alpha\left(g_{n}^{-1}\right) \alpha\left(f_{n}\right)\right|=o_{\omega}\left(d_{n}\right)$. Let $s_{n}$ be a shortest preimage of $\alpha\left(g_{n}^{-1} f_{n}\right)$ in $G$. Then $\left|s_{n}\right|=o_{\omega}\left(d_{n}\right)$ and $u_{n}=f_{n}^{-1} g_{n} s_{n} \in N$. Note that $\left|u_{n}\right| \leq\left|f_{n}\right|+\left|g_{n}\right|+\left|s_{n}\right|=O_{\omega}\left(d_{n}\right)$. Hence $u=\left(u_{n}\right)^{\omega} \in \operatorname{Con}^{\omega}(N, d)$. Clearly $\gamma u=\left(g_{n} s_{n}\right)^{\omega}=\left(g_{n}\right)^{\omega}$. Thus $\mathcal{F}_{b} \subseteq \gamma \operatorname{Con}^{\omega}(N, d)$ and the second assertion is proved.

Similarly it is easy to show that each subset of the form $\gamma \operatorname{Con}^{\omega}(N, d)$, where $\gamma \in$ $G_{e}^{\omega}(d)$, is a fiber. This and the second assertion imply the third one.

Given two elements $\left(g_{n}\right)^{\omega}$ and $\left(h_{n}\right)^{\omega}$ of $\mathcal{F}_{b}$, the element $\left(h_{n} g_{n}^{-1}\right)^{\omega}$ belongs to $N_{e}^{\omega}(d)$ and takes $\left(g_{n}\right)^{\omega}$ to $\left(h_{n}\right)^{\omega}$. This proves the fourth assertion. 
Finally, assume that a ball of radius $\varepsilon \in(0, \infty]$ in $\operatorname{Con}^{\omega}(N, d)$ consists of a single point. Let $h=\left(h_{n}\right)^{\omega}$ and $g=\left(g_{n}\right)^{\omega}$ be two elements of $\operatorname{Con}^{\omega}(G, d)$ such that $\operatorname{dist}(g, h)<\varepsilon / 2$. Note that

$$
\left|\sigma\left(g_{n}^{-1} h_{n} N\right)\right| \leq\left|g_{n}^{-1} h_{n}\right|<\varepsilon d_{n} / 2
$$

$\omega$-almost surely. Then for $u_{n}=g_{n}^{-1} h_{n}\left(\sigma\left(g_{n}^{-1} h_{n} N\right)\right)^{-1}$, we have $\left|u_{n}\right|<\varepsilon d_{n} \omega-$ almost surely. Since $u_{n} \in N,\left(u_{n}\right)^{\omega}$ belongs to the ball of radius $\varepsilon$ in $\operatorname{Con}^{\omega}(N, d)$ around $(1)^{\omega}$. Hence $\left(u_{n}\right)^{\omega}=(1)^{\omega}$, ie, $\left|u_{n}\right|=o_{\omega}\left(d_{n}\right)$. Finally we obtain

$$
\begin{aligned}
\operatorname{dist}(\hat{\alpha}(g), \hat{\alpha}(h)) & =\stackrel{\omega}{\lim } d\left(h_{n} N, g_{n} N\right) / d_{n}=\stackrel{\omega}{\lim } d\left(g_{n}^{-1} h_{n} N, N\right) / d_{n} \\
& =\stackrel{\omega}{\lim }\left|\sigma\left(g_{n}^{-1} h_{n} N\right)\right| / d_{n} \leq \stackrel{\omega}{\lim }\left(\left|g_{n}^{-1} h_{n}\right|+\left|u_{n}\right|\right) / d_{n} \\
& =\stackrel{\omega}{\lim }\left|g_{n}^{-1} h_{n}\right| / d_{n}=\operatorname{dist}(g, h) .
\end{aligned}
$$

Thus the restriction of $\hat{\alpha}$ to any ball of radius $\varepsilon / 4$ is an isometry.

Recall that a subspace $Y$ of a metric space $X$ is said to be convex if any geodesic path $p$ in $X$ such that $p_{ \pm} \in Y$ belongs to $Y$. In particular, if $X$ is geodesic, then any convex subspace of $X$ is geodesic.

Proposition 5.3 Let $d=\left(d_{n}\right)$ a scaling sequence, $\omega$ a nonprincipal ultrafilter. Suppose that $T$ is a convex $\mathbb{R}$-tree in $\operatorname{Con}^{\omega}(G, d)$. Then for any point $y \in \operatorname{Con}^{\omega}(G, d)$ such that $\widehat{\alpha}(y) \in T$, there is an isometric section $\sigma: T \rightarrow \operatorname{Con}^{\omega}(G, d)$ of $\widehat{\alpha}$ such that $y \in \sigma(T)$.

To prove the proposition we need an auxiliary result.

Lemma 5.4 For any point $x \in \operatorname{Con}^{\omega}(G, d)$ and any fiber $\mathcal{F}_{b}$, there is a point $f \in \mathcal{F}_{b}$ such that $\operatorname{dist}\left(x, \mathcal{F}_{b}\right)=\operatorname{dist}(x, f)=\operatorname{dist}(\widehat{\alpha}(x), b)$.

Proof By Theorem 5.2 (c), it suffices to prove the statement of the lemma for $x=(1)^{\omega}$. Let $\mathcal{F}=\gamma \operatorname{Con}^{\omega}(N, d)$, where $\gamma=\left(g_{n}\right) \in G_{e}^{\omega}(d)$. We fix any section $s: G / N \rightarrow G$ that assigns to every element of $G / N$ a shortest preimage. Take $f=\left(s\left(g_{n} N\right)\right)^{\omega}$. By the choice of $s$, we have $\left|s\left(g_{n} N\right)\right|=\left|\alpha\left(s\left(g_{n} N\right)\right)\right|$. Hence

$$
\operatorname{dist}(1, f)=\operatorname{dist}\left(1,\left(\alpha \circ s\left(g_{n} N\right)\right)^{\omega}\right)=\operatorname{dist}(1, \widehat{\alpha}(f))=\operatorname{dist}(1, b) .
$$

Note that $\gamma^{-1} f=\left(g_{n}^{-1} s\left(g_{n} N\right)\right)^{\omega} \in \operatorname{Con}^{\omega}(N, d)$ and thus $f \in \mathcal{F}$. Finally for any point $f^{\prime} \in \mathcal{F}$ we have $f^{\prime}=\left(g_{n} u_{n}\right)^{\omega}$ for some $u_{n} \in N$. Hence

$$
\operatorname{dist}\left(1, f^{\prime}\right)=\lim \frac{\left|g_{n} u_{n}\right|}{d_{n}} \geq \lim \frac{\left|s\left(g_{n} N\right)\right|}{d_{n}}=\operatorname{dist}(1, f) .
$$

Thus $\operatorname{dist}(1, \mathcal{F})=\operatorname{dist}(1, f)=\operatorname{dist}(1, b)$. 
Proof of Proposition 5.3 We first consider a segment $I=[a, b] \subset T$ and any preimage $x$ of $a$. Let $f$ be the point of $\mathcal{F}_{b}$ provided by Lemma 5.4. Recall that $\operatorname{Con}^{\omega}(G, d)$ is a geodesic metric space. Let $J_{I}=[x, f]$ be a geodesic segment in $\operatorname{Con}^{\omega}(G, d)$. Note that $\hat{\alpha}$ does not increase the distance. If $u, v \in J_{I}$ and $\operatorname{dist}(\alpha(u), \alpha(v))<\operatorname{dist}(u, v)$, then

$$
\begin{aligned}
\operatorname{dist}(a, b) & \leq \operatorname{dist}(a, \alpha(u))+\operatorname{dist}(\alpha(u), \alpha(v))+\operatorname{dist}(\alpha(v), b) \\
& <\operatorname{dist}(x, u)+\operatorname{dist}(u, v)+\operatorname{dist}(v, f)=\left|J_{I}\right|
\end{aligned}
$$

that contradicts the choice of $f$. Thus $\hat{\alpha}$ isometrically maps $J_{I}$ to its image in $\operatorname{Con}^{\omega}(G / N, d)$. Since $\left|J_{I}\right|=\operatorname{dist}(x, f)=\operatorname{dist}(a, b)=|I|$ by the choice of $f, \hat{\alpha}\left(J_{I}\right)$ is a geodesic segment in $\operatorname{Con}^{\omega}(G / N, d)$. As $T$ is convex, we have $\widehat{\alpha}\left(J_{I}\right) \in T$. Hence $\hat{\alpha}\left(J_{I}\right)=I$. Thus for any preimage $x$ of $a$, there is an isometric section $\sigma_{I}: I \rightarrow \operatorname{Con}^{\omega}(G, d)$ such that $\sigma_{I}(a)=x$.

Let us fix a vertex $o$ of $T$. Suppose that we have already found an isometric section $\sigma_{T_{0}}$ for a subtree $T_{0} \subset T$ containing $o$ such that $y \in T_{0}$. Let $b \in T \backslash T_{0}$. Then there is a unique point $a \in T_{0}$ such that $[b, o] \cap T_{0}=[a, o]$. Let $x=\sigma_{T_{0}}(a)$ and let $\sigma_{I}: I \rightarrow \operatorname{Con}^{\omega}(G, d)$ be an isometric section for $I=[a, b]$ such that $\sigma_{I}(a)=x$. We then define an isometric section $\sigma_{T_{1}}: T_{1} \rightarrow \operatorname{Con}^{\omega}(G, d)$, where $T_{1}=T_{0} \cup[a, b]$, by the rule

$$
\sigma_{T_{1}}(t)= \begin{cases}\sigma_{T_{0}}(t), & \text { if } t \in T_{0}, \\ \sigma_{I}(t), & \text { if } t \in[a, b] .\end{cases}
$$

Now we can complete the proof by transfinite induction.

Corollary 5.5 If $\operatorname{Con}^{\omega}(G / N, d)$ is an $\mathbb{R}$-tree for some scaling sequence $d=\left(d_{n}\right)$ and nonprincipal ultrafilter $\omega$, then there is an isometric section $\sigma: \operatorname{Con}^{\omega}(G / N, d) \rightarrow$ $\operatorname{Con}^{\omega}(G, d)$ of the map $\hat{\alpha}$.

\subsection{Central extensions of lacunary hyperbolic groups}

We keep the notation from the previous section here. Given a product $X \times Y$ of metric spaces $X$ and $Y$, by the product metric we mean the metric on $X \times Y$ defined by the rule

$$
\operatorname{dist}_{X \times Y}\left(\left(x_{1}, y_{1}\right),\left(x_{2}, y_{2}\right)\right)=\operatorname{dist}_{X}\left(x_{1}, x_{2}\right)+\operatorname{dist}_{Y}\left(y_{1}, y_{2}\right) \text {. }
$$

Recall that every 2-dimensional cohomology class on a hyperbolic group can be represented by a bounded cocycle by Neumann and Reeves [37] (see also Mineyev [36], where it is proved for all dimensions $\geq 2$ ). This implies that for any finitely generated group $G$ and any finitely generated central subgroup $N \leq G$ such that $G / N$ is hyperbolic, $G$ is quasi-isometric to $N \times G / N$ [37]. (A particular case was also proved 
in Gersten [17].) Therefore, for any $d$ and $\omega, \operatorname{Con}^{\omega}(G, d)$ is bi-Lipschitz equivalent to $\operatorname{Con}^{\omega}(N, d) \times \operatorname{Con}^{\omega}(G / N, d)$ endowed with the product metric. In this section we generalize this result to the class of lacunary hyperbolic groups as follows.

Theorem 5.6 Let $N$ be a central subgroup of a finitely generated group $G$ endowed with the induced metric. Suppose that for some nonprincipal ultrafilter $\omega$ and some scaling sequence $d=\left(d_{n}\right)$, $\operatorname{Con}^{\omega}(G / N, d)$ is an $\mathbb{R}$-tree. Then $\operatorname{Con}^{\omega}(G, d)$ is biLipschitz equivalent to $\operatorname{Con}^{\omega}(N, d) \times \operatorname{Con}^{\omega}(G / N, d)$ endowed with the product metric.

Proof Let $\sigma: \operatorname{Con}^{\omega}(G / N, d) \rightarrow \operatorname{Con}^{\omega}(G, d)$ be the isometric section provided by Corollary 5.5. We define a map $\varkappa$ : $\operatorname{Con}^{\omega}(N, d) \times \operatorname{Con}^{\omega}(G / N, d) \rightarrow \operatorname{Con}^{\omega}(G, d)$ as follows. Suppose that $x \in \operatorname{Con}^{\omega}(G / N, d)$ and $g=\left(g_{n}\right)^{\omega} \in \operatorname{Con}^{\omega}(N, d)$, where $\left(g_{n}\right) \in N_{e}^{\omega}(d)$. Then $x(g, x)=\left(g_{n}\right) \sigma(x)$.

First observe that $\varkappa$ is well-defined. Indeed if $\left(g_{n}\right)^{\omega}=\left(h_{n}\right)^{\omega} \in \operatorname{Con}^{\omega}(N, d)$, then $g_{n}=h_{n} u_{n}$, where $u_{n} \in N$ and $\left|u_{n}\right|=o_{\omega}\left(d_{n}\right)$. Since $N$ is central, for any $y=$ $\left(y_{n}\right)^{\omega} \in \operatorname{Con}^{\omega}(G, d)$, we have

$$
\left(g_{n}\right)\left(y_{n}\right)^{\omega}=\left(h_{n} u_{n} y_{n}\right)^{\omega}=\left(h_{n} y_{n} u_{n}\right)^{\omega}=\left(h_{n} y_{n}\right)^{\omega}=\left(h_{n}\right)\left(y_{n}\right)^{\omega} .
$$

Further observe that for any $y=\left(y_{n}\right)^{\omega} \in \operatorname{Con}^{\omega}(G, d)$ and $g_{1}=\left(g_{1 n}\right)^{\omega}, g_{2}=\left(g_{2 n}\right)^{\omega} \in$ $\operatorname{Con}^{\omega}(N, d)$ we have

$$
\operatorname{dist}\left(g_{1 n}, g_{2 n}\right)=\operatorname{dist}\left(y_{n} g_{1 n}, y_{n} g_{2 n}\right)=\operatorname{dist}\left(g_{1 n} y_{n}, g_{2 n} y_{n}\right) .
$$

Therefore,

$$
\operatorname{dist}\left(g_{1}, g_{2}\right)=\operatorname{dist}\left(\left(g_{1 n}\right) y,\left(g_{2 n}\right) y\right) .
$$

Suppose now that $\left(g_{1}, x_{1}\right),\left(g_{2}, x_{2}\right) \in \operatorname{Con}^{\omega}(N, d) \times \operatorname{Con}^{\omega}(G / N, d)$, where $g_{1}=$ $\left(g_{1 n}\right)^{\omega}, g_{2}=\left(g_{2 n}\right)^{\omega} \in \operatorname{Con}^{\omega}(N, d)$, and $k_{1}=\varkappa\left(g_{1}, x_{1}\right), k_{2}=\varkappa\left(g_{2}, x_{2}\right)$. Applying (24) we obtain

$$
\begin{aligned}
\operatorname{dist}\left(k_{1}, k_{2}\right) & =\operatorname{dist}\left(\left(g_{1 n}\right) \sigma\left(x_{1}\right),\left(g_{2 n}\right) \sigma\left(x_{2}\right)\right) \\
& \leq \operatorname{dist}\left(\left(g_{1 n}\right) \sigma\left(x_{1}\right),\left(g_{2 n}\right) \sigma\left(x_{1}\right)\right)+\operatorname{dist}\left(\left(g_{2 n}\right) \sigma\left(x_{1}\right),\left(g_{2 n}\right) \sigma\left(x_{2}\right)\right) \\
& =\operatorname{dist}\left(g_{1}, g_{2}\right)+\operatorname{dist}\left(\sigma\left(x_{1}\right), \sigma\left(x_{2}\right)\right) \\
& =\operatorname{dist}\left(g_{1}, g_{2}\right)+\operatorname{dist}\left(x_{1}, x_{2}\right)
\end{aligned}
$$

Note that $\widehat{\alpha}(\varkappa(g, x))=\hat{\alpha}(\sigma(x))=x$ since the action of $G_{e}^{\omega}(d)$ preserves fibers. Hence

$$
\operatorname{dist}\left(x_{1}, x_{2}\right)=\operatorname{dist}\left(\widehat{\alpha}\left(k_{1}\right), \widehat{\alpha}\left(k_{2}\right)\right) \leq \operatorname{dist}\left(k_{1}, k_{2}\right) .
$$


Now reversing the inequality and replacing pluses with minuses in (25), we obtain

$$
\operatorname{dist}\left(g_{1}, g_{2}\right) \leq \operatorname{dist}\left(k_{1}, k_{2}\right)+\operatorname{dist}\left(x_{1}, x_{2}\right) \leq 2 \operatorname{dist}\left(k_{1}, k_{2}\right) .
$$

Finally combining (26) and (27) we obtain

$$
\operatorname{dist}\left(\left(g_{1}, x_{1}\right),\left(g_{2}, x_{2}\right)\right)=\operatorname{dist}\left(g_{1}, g_{2}\right)+\operatorname{dist}\left(x_{1}, x_{2}\right) \leq 3 \operatorname{dist}\left(k_{1}, k_{2}\right) .
$$

This inequality together with (25) shows that $\varkappa$ is a 3-bi-Lipschitz map. To complete the proof it remains to note that $\varkappa$ is surjective by Theorem 5.2 (d).

Our next goal is to prove Theorem 5.8, which will be used in the next section. We start with the following general lemma.

Lemma 5.7 Let $G$ be a non-virtually cyclic finitely generated group, $\mathcal{C}=\operatorname{Con}^{\omega}(G, d)$ its asymptotic cone. Let $X \subseteq \mathcal{C}$ be a finite subset. Then for every $x \in \mathcal{C} \backslash X$ there exists a path $p$ in $\mathcal{C} \backslash X$ with $p_{-}=x$, containing points arbitrary far away from $x$.

Proof Since $G$ is finitely generated, there exists an infinite geodesic ray in the Cayley graph of $G$. Its ultralimit is an infinite geodesic ray $r$ in $\mathcal{C}$. Since $\mathcal{C}$ is homogeneous, we can assume that $r_{-}=x$. We can also assume that $x=e=(1)^{\omega}$.

Let $X=\left\{x_{1}, \ldots, x_{m}\right\}, x_{i}=\left(x_{i}(n)\right)^{\omega}$. Let $2 l=\min \left\{\operatorname{dist}\left(e, x_{i}\right), i=1, \ldots, m\right\}$, $l_{i}=\operatorname{dist}\left(e, x_{i}\right)$. For every $g=\left(g_{n}\right) \in G_{e}^{\omega}(d)$ with $\left|g_{n}\right| \leq l d_{n}$ consider the ray $g^{-1} r$. The union of $g^{-1} r$ and any geodesic $\left[e, g^{-1} e\right]$ is a path $p(g)$ starting at $e$ and containing points arbitrary far from $e$.

If one of these paths does not contain any $x_{i}$, we are done. Assume that every $p(g)$ contains $x_{i}$ from $X$. For every $i=1, \ldots, m$ let $M_{i}$ be the set of all $g=\left(g_{n}\right)^{\omega}$ with $\left|g_{n}\right| \leq l d_{n}$ such that $p(g)$ contains $x_{i}$. Then the union of $M_{i}$ contains the limit $\lim ^{\omega} \operatorname{Ball}_{G}\left(e, l d_{n}\right)$.

For every $g=\left(g_{n}\right)^{\omega} \in M_{i} g^{-1} r$ must contain $x_{i}$ because $\left[e, g^{-1} e\right]$ is too short to contain $x_{i}$. Hence $g x_{i} \in r$. Since $\operatorname{dist}(e, g e) \leq l$, $\operatorname{dist}\left(g x_{i}, g e\right)=\operatorname{dist}\left(x_{i}, e\right)=l_{i}$, we conclude that $g x_{i}$ belongs to the subgeodesic $r\left[l_{i}-l, l_{i}+l\right]$ of the ray $r$. Pick a number $N \geq 2$ and divide the interval $r\left[l_{i}-l, l_{i}+l\right]$ into $N+1$ equal subintervals. Let $a_{1}, \ldots, a_{N}$ be the division points, $a_{j}=\left(a_{j}(n)\right)^{\omega}$. Then for every $g=\left(g_{n}\right)^{\omega} \in M_{i}$ the point $g x_{i}$ is within distance $2 l /(N+1)$ from one of $a_{j}$. Hence

$$
\operatorname{dist}_{G}\left(g_{n} x_{i}(n), a_{j}(n)\right) \leq \frac{2 l d_{n}}{N+1}+o_{\omega}\left(d_{n}\right) \leq \frac{2 l d_{n}}{N}
$$

$\omega$-almost surely. Therefore $g_{n} x_{i}(n)$ is in the ball $B_{j}$ of radius $2 l d_{n} / N$ around $a_{j}(n)$ in $G \omega$-almost surely. Hence, for any fixed $i \in\{1, \ldots, m\}$, the number of elements 
$g_{n}$ such that $g_{n} x_{i}(n) \in B_{j}$ does not exceed $N$ times the number of elements of the ball $\operatorname{Ball}_{G}\left(2 l d_{n} / N\right)$.

Let $f(n)$ be the growth function of the group $G$. Since the union of $M_{i}$ contains the limit $\lim ^{\omega} \operatorname{Ball}_{G}\left(l d_{n}\right)$, we proved in the previous paragraph that for every $N \geq 2$,

$$
f\left(l d_{n}\right) \leq m N f\left(\frac{l d_{n}}{N}\right)
$$

$\omega$-almost surely.

By Gromov [19, page 68] (see also van den Dries and Wilkie [9]), then the asymptotic cone $\operatorname{Con}\left(G,\left(l d_{n}\right)\right)$ is locally compact. As shown by Point [48], that asymptotic cone has Minkovski dimension 1 (for the definition, see [48]). Then by [48] $G$ is virtually nilpotent and $\operatorname{Con}^{\omega}\left(G,\left(l d_{n}\right)\right)$ is homeomorphic to $\mathbb{R}$. But then [13, Proposition 6.1] implies that $G$ is virtually cyclic, which contradicts the assumption of the lemma. $\square$

For every metric space $X$, we define a connectedness degree $c(X)$ as the minimal number of points of $X$ whose removal disconnects $X$. If $X$ can not be disconnected by removing finitely many points, we set $c(X)=\infty$. In particular, $X$ has cut points if and only if $c(X)=1$. By a cut set of $X$ we mean any subset of $X$ whose removal disconnects $X$.

Theorem 5.8 Let $N$ be a central subgroup of a finitely generated group $G$. Suppose that $\operatorname{Con}^{\omega}(N, d)$ consists of $m<\infty$ points for some nonprincipal ultrafilter $\omega$ and some scaling sequence $d=\left(d_{n}\right)$. Then

$$
c\left(\operatorname{Con}^{\omega}(G, d)\right)=m c\left(\operatorname{Con}^{\omega}(G / N, d)\right) .
$$

Moreover, a finite subset $C \subset \operatorname{Con}^{\omega}(G, d)$ disconnects $\operatorname{Con}^{\omega}(G, d)$ if and only if $C$ contains a full preimage of a cut set of $\operatorname{Con}^{\omega}(G / N, d)$ under the map $\widehat{\alpha}: \operatorname{Con}^{\omega}(G, d) \rightarrow$ $\operatorname{Con}^{\omega}(G / N, d)$.

Proof First note that $G$ is not virtually cyclic. Indeed otherwise either $N$ is finite or $N$ contains an infinite cyclic group. In the first case $\operatorname{Con}^{\omega}(G, d)$ is $\mathbb{R}, m=1$, and the proposition is obvious. In the second case $\operatorname{Con}^{\omega}(N, d)$ contains infinitely many points that contradicts $m<\infty$.

Take a finite set $C$ in $\operatorname{Con}^{\omega}(G, d)$. Let $\widehat{C}$ be the full preimage of $\widehat{\alpha}(C)$ under $\widehat{\alpha}$. Then $\widehat{C}$ is finite.

We shall need the following statement.

Lemma 5.9 Let $a, a^{\prime} \in \operatorname{Con}^{\omega}(G, d) \backslash \widehat{C}$ and $\widehat{\alpha}(a)=\widehat{\alpha}\left(a^{\prime}\right)$. Then $a$ and $a^{\prime}$ are in the same connected component of $\operatorname{Con}^{\omega}(G, d) \backslash \widehat{C}$. 
Proof Let $\gamma \in N_{e}^{\omega}(d)$ be such that $\gamma a=a^{\prime}$ (such $\gamma$ exists by Theorem 5.2). By Lemma 5.7, there exists a path $r$ in $\operatorname{Con}^{\omega}(G, d) \backslash \widehat{C}$ with $r_{-}=a$ containing points arbitrary far away from $a$. Consider the path $r^{\prime}=\gamma r$. Then $r_{-}^{\prime}=a^{\prime}$. Note that $\widehat{C}$ is closed under the action of $N_{e}^{\omega}(d)$. Hence $r^{\prime}$ does not contain points from $\widehat{C}$. Consider a point $z$ on $r$ such that $\operatorname{dist}(z, C)>\operatorname{dist}\left(a, a^{\prime}\right)$. Let $z^{\prime}=\gamma z$ (using Theorem 5.2 again). Since $\operatorname{Con}^{\omega}(G, d)$ is homogeneous, there exists $\beta \in G_{e}^{\omega}(d)$ such that $z=\beta a$. Since $N$ is central in $G$, we have

$$
\operatorname{dist}\left(z, z^{\prime}\right)=\operatorname{dist}(\beta a, \gamma \beta a)=\operatorname{dist}(\beta a, \beta \gamma a)=\operatorname{dist}\left(a, a^{\prime}\right) .
$$

Therefore any geodesic path from $z$ to $z^{\prime}$ avoids $\widehat{C}$.

Now consider the path $w$ that goes first from $a$ to $z$ along $r$, then from $z$ to $z^{\prime}$ along any geodesic $\left[z, z^{\prime}\right]$, then goes back to $a^{\prime}$ along $r^{\prime}$. That path avoids points from $\widehat{C}$.

Let us continue the proof of Theorem 5.8.

Suppose the finite set $C$ does not contain a full preimage of a cut set of $\operatorname{Con}^{\omega}(G / N, d)$ under $\widehat{\alpha}$. We need to show that $C$ is not a cut set of $\operatorname{Con}^{\omega}(G, d)$ that is any two points $u, v \in \operatorname{Con}^{\omega}(G, d) \backslash C$ can be connected by a path avoiding $C$. Take any geodesic $p$ connecting $u, v \in \operatorname{Con}^{\omega}(G, d) \backslash C$. If this geodesic does not contain points in $C$, we are done. Suppose that $p$ contains a point from $C$. Then it is enough to show how to replace subpaths of $p$ connecting points close enough to points in $C$. Thus without loss of generality we can assume that $u$ and $v$ are from $\operatorname{Ball}(c, \zeta) \backslash \widehat{C}$ for some $c \in C$ and some small enough $\zeta$.

Pick any $\zeta$ such that $\operatorname{Ball}(c, \zeta) \cap \widehat{C}=\{c\}$. Pick two points $u, v \in \operatorname{Ball}(c, \zeta) \backslash\{c\}$. Then $u, v \notin \widehat{C}$.

Suppose that $\hat{\alpha}(C)$ is not a cut set of $\operatorname{Con}^{\omega}(G / N, d)$. Then $\hat{\alpha}(u)$ can be connected with $\hat{\alpha}(v)$ by a path $q$ in $\operatorname{Con}^{\omega}(G / N, d) \backslash \hat{\alpha}(C)$. Since $\hat{\alpha}$ is a covering map by Theorem 5.2 (e), we can lift the path $p$ to a path $\hat{p}$ in $\operatorname{Con}^{\omega}(G, d)$ avoiding $\widehat{C}$ and such that $\hat{p}_{-}=u$. Note that $\hat{\alpha}\left(\hat{p}_{+}\right)=\hat{\alpha}(v)$. Hence by Lemma 5.9, we can connect $\hat{p}_{+}$with $v$ by a path $p_{1}$ in $\operatorname{Con}^{\omega}(G, d) \backslash \widehat{C}$. The composition of $\hat{p}$ and $p_{1}$ connects $u$ and $v$ and avoids $C$ as required.

Now suppose that $\widehat{\alpha}(C)$ is a cut set in $\operatorname{Con}^{\omega}(G / N, d)$. Let $C^{\prime}$ be the union of all fibers of $\hat{\alpha}$ contained in $C$. We can assume that $\widehat{\alpha}\left(C^{\prime}\right)$ is not a cut set. Therefore there exists a path from $\hat{\alpha}(u)$ to $\hat{\alpha}(v)$ in $\operatorname{Con}^{\omega}(G / N, d) \backslash \hat{\alpha}\left(C^{\prime}\right)$. That path must contain points $\hat{\alpha}\left(c_{1}\right)$ for some $c_{1} \in C$ with $c_{1} \notin C^{\prime}$. Lifting this path to $\operatorname{Con}^{\omega}(G, d)$ and using Lemma 5.9, we can obtain a path connecting $u$ and $v$ in $\operatorname{Con}^{\omega}(G, d) \backslash C^{\prime}$ but containing points in $C \backslash C^{\prime}$. If we could replace parts of this path connecting points close the points in $C \backslash C^{\prime}$ by paths avoiding these points we would show that $C$ is not 
a cut set. Thus without loss of generality we can assume that $u, v$ are very close to a point in $C \backslash C^{\prime}$, ie we can assume that $c \in C \backslash C^{\prime}$.

Then there exists a point $c^{\prime} \notin C$ with $\hat{\alpha}\left(c^{\prime}\right)=\hat{\alpha}(c)$. Let $\gamma \in N_{e}^{\omega}(d)$ be such that $\gamma c=c^{\prime}$. We can assume that $\zeta$ is small enough so that $\gamma \operatorname{Ball}(c, \zeta)$ does not intersect $C$. By Lemma 5.9, there exist paths $r, r^{\prime}$ in $\operatorname{Con}^{\omega}(G, d) \backslash \hat{C}$ with $r_{-}=u, r_{+}=\gamma u$, $r_{-}^{\prime}=v, r_{+}^{\prime}=\gamma v$. Then one can travel from $u$ to $v$ by first going to $\gamma u$ along $r$ then by a path from $\gamma u$ to $\gamma v$ inside $\gamma \operatorname{Ball}(c, \zeta)$, then back to $v$ along $r^{\prime}$. That path avoids $C$. Hence $C$ is not a cut set in $\operatorname{Con}^{\omega}(G, d)$.

We have proved that every cut set of $\operatorname{Con}^{\omega}(G / N, d)$ contains a full preimage of a cut set of $\operatorname{Con}^{\omega}(G / N, d)$. This implies

$$
c\left(\operatorname{Con}^{\omega}(G, d)\right) \geq m c\left(\operatorname{Con}^{\omega}(G / N, d)\right) .
$$

In order to prove the opposite inequality, note that if a finite set $\left\{b_{1}, \ldots, b_{c}\right\}$ disconnects $\operatorname{Con}^{\omega}(G / N, d)$ then the union of fibers $\mathcal{F}_{b_{1}}, \ldots, \mathcal{F}_{b_{c}}$ disconnect $\operatorname{Con}^{\omega}(N, d)$ because $\hat{\alpha}$ is continuous.

\subsection{Applications}

Let us consider a family of central extensions of a lacunary hyperbolic group constructed as follows. By Corollary 4.15 and Remark 4.8 there is a presentation

$$
H=\left\langle a, b \mid \bigcup_{i=1}^{\infty} \mathcal{R}_{i}\right\rangle
$$

that simultaneously satisfies the graded small cancellation condition and the classical small cancellation condition $C^{\prime}(1 / 24)$, where for every $i, \mathcal{R}_{i}$ consists of cyclic shifts of a single word $R_{i}$ and its inverses. Throughout this section we fix any such presentation and denote by $r_{n}$ the length of the word $R_{n}$. Given a sequence of integers $k=\left(k_{n}\right)$, where $k_{n} \geq 2$, we consider the central extension of $H$ defined as follows.

$$
G(k)=\left\langle a, b \mid\left[R_{n}, a\right]=1,\left[R_{n}, b\right]=1, R_{n}^{k_{n}}=1, n=1,2, \ldots\right\rangle
$$

We begin with auxiliary results.

Lemma 5.10 Let $U$ be a subword of a word $R_{n}^{k_{n}}$ of length at most $k_{n} r_{n} / 2$. Then the length of the element represented by the word $U$ in $G$ is at least $|U| / 8$.

Proof Suppose that $U=V$ in $G$ where the word $V$ is geodesic in $G$. Let $\Delta$ be a diagram over $G$ corresponding to this equality. Let $\partial \Delta=p q^{-1}$ be the decomposition of the boundary of $\Delta$, where $U \equiv \operatorname{Lab}(p), V \equiv \operatorname{Lab}(q)$. We can turn $\Delta$ into a 
diagram $\Delta^{\prime}$ over $H$ with the same boundary label by the following procedure. Every cell corresponding to the relation $R_{i}^{k_{i}}$ is replaced by a union of $k_{i}$ cells each labeled by $R_{i}$ connected by a point. Every cell labeled by $\left[R_{i}, a\right]$ or $\left[R_{i}, b\right]$ is replaced by the union of two cells labeled by $R_{i}$ connected by an edge labeled by $a$ or $b$. In both cases the union has the same boundary label as the original cell (see Figure 6).
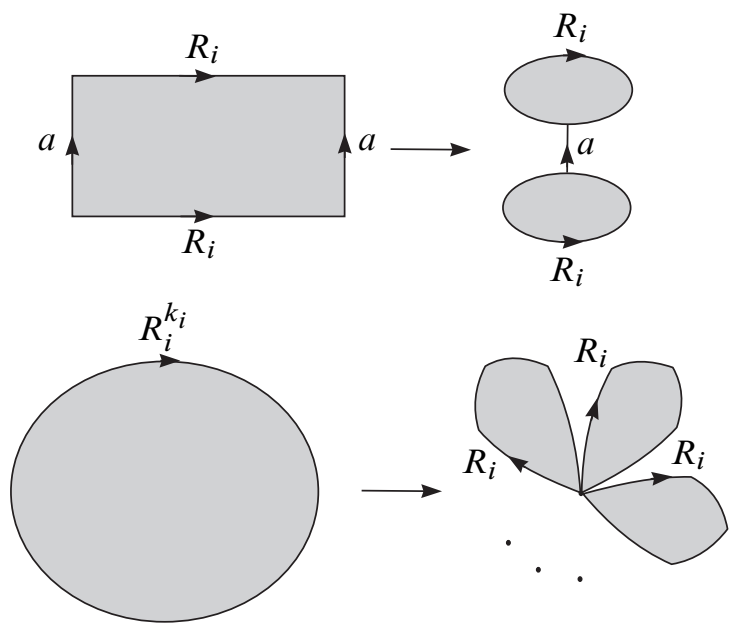

Figure 6: Transforming cells in $\Delta$

To any cell $\Pi$ labeled by $R_{n}^{ \pm 1}$ in $\Delta^{\prime}$, we assign the number -1 if $R_{n}$ reads along $\partial \Pi$ in the clockwise direction and 1 otherwise. By the algebraic number of $R_{n}$-cells in $\Delta^{\prime}$ we mean the sum of the assigned numbers over all cells labeled by $R_{n}^{ \pm 1}$. Notice that the algebraic number of $R_{n}$-cells in $\Delta^{\prime}$ is divisible by $k_{n}$. If we reduce $\Delta^{\prime}$ by canceling pairs of cells having a common edge and being mirror images of each other, we obtain a diagram $\Delta^{\prime \prime}$ with the same property. We keep the notation $p q^{-1}$ for the boundary of $\Delta^{\prime \prime}$. We have to show that

$$
|q| \geq|p| / 8 \text {. }
$$

Assume that the boundary of some cell $\Pi$ in $\Delta^{\prime \prime}$ has a common subpath of length at least $|\partial \Pi| / 24$ with $p$. Then $\partial \Pi$ is labeled by $R_{n}$ by the $C^{\prime}(1 / 24)-$ condition, and since $p$ is labeled by a power of $R_{n}$ we may assume without loss of generality that $\partial \Pi \subset p$. After cutting such a cell off we obtain a new diagram with boundary $p^{\prime} q^{-1}$, where $\operatorname{Lab}\left(q^{\prime}\right)$ is again a power of $R_{n}$. Continuing this process we obtain a subdiagram $\Sigma$ of $\Delta^{\prime \prime}$ with boundary $t q^{-1}$, where $t$ is labeled by a power of $R_{n}$, such that no $R_{n}$-cell $\Pi$ of $\Sigma$ has a common subpath of lengths at least $|\partial \Pi| / 24$ with $t$. Now there are two cases to consider. 
Case 1 Suppose that $|t| \geq|p| / 4$. Observe that any cell $\Pi$ in $\Sigma$ satisfies the following condition:

$(+)\left\{\begin{array}{l}\text { For any two vertices } x, y \in t \cap \partial \Pi, \text { there is a common subpaths of } \partial \Pi \\ \text { and } t \text { connecting } x \text { and } y\end{array}\right.$

Indeed otherwise there is a subdiagram $\Xi$ of $\Sigma$ such that $\Xi$ contains at least one cell and $\partial \Xi=s_{1} s_{2}$, where $s_{1}$ is a subpath of $t$ and $s_{2}$ is a subpath of $\partial \Pi$. Note that a common subpath of the boundary of any cell $\Omega$ in $\Xi$ and $s_{1}$ (respectively $s_{2}$ ) has length less than $|\partial \Omega| / 24$ by the construction of $\Sigma$ (respectively since $\Delta^{\prime \prime}$ is reduced). This contradicts Lemma 3.7.

In particular, $(+)$ and the $C^{\prime}(1 / 24)$-condition imply that for any cell $\Pi$ of $\Sigma$, the intersection $\partial \Pi \cap t$ is a path of length less than $|\partial \Pi| / 24$. Note that if the number of common edges of $t$ and $q$ is at least $|p| / 8$, the inequality (29) is obvious. Hence we may assume that more than $|t|-|p| / 8 \geq|p| / 8$ edges of $t$ belong to cells of $\Sigma$. Therefore the sum of perimeters of all cells in $\Sigma$ is greater than $24|p| / 8=3|p|$. Applying Lemma 3.8 to $\Delta^{\prime \prime}$ we obtain $|p|+|q| \geq 9|p| / 4$, which yields (29).

Case 2 Suppose that $|t| \leq|p| / 4$. This means that we have to cut at least $k=$ $3|p| /\left(4 r_{n}\right) R_{n}$-cells to get $\Sigma$ from $\Delta^{\prime \prime}$. Note that $k \leq|p| / r_{n} \leq k_{n} / 2$ and all these cells have the same orientation by the $C^{\prime}(1 / 24)$-condition. Since the algebraic number of $R_{n}$-cells in $\Delta^{\prime \prime}$ should be divisible by $k_{n}$, the total number of $R_{n}$-cells in $\Delta^{\prime \prime}$ is at least $2 k$. Applying Lemma 3.8 again, we obtain $|p|+|q| \geq 6 k r_{n} / 4 \geq 9|p| / 8$. Hence $|q| \geq|p| / 8$.

Theorem 5.11 For every $m \geq 2$ there exists a finitely generated group $G$ such that for any ultrafilter $\omega$ and any scaling sequence $d=\left(d_{n}\right)$, exactly one of the following possibilities occurs and both of them can be realized for suitable $\omega$ and $d$.

(a) $\operatorname{Con}^{\omega}(G, d)$ is an $m$-fold cover of a circle-tree and $c\left(\operatorname{Con}^{\omega}(G, d)\right)=m$. Moreover, a finite subset $C \subset \operatorname{Con}^{\omega}(G, d)$ disconnects $\operatorname{Con}^{\omega}(G, d)$ if and only if $C$ contains a fiber of the map $\widehat{\alpha}: \operatorname{Con}^{\omega}(G, d) \rightarrow \operatorname{Con}^{\omega}(G / N, d)$.

(b) $\operatorname{Con}^{\omega}(G, d)$ is an $\mathbb{R}$-tree.

In particular, in both cases $\operatorname{Con}^{\omega}(G, d)$ is locally isometric to an $\mathbb{R}$-tree.

Proof Let $G=G(k)$ be the group corresponding to the sequence $k_{n}=m \geq 2$ for all $n$. The central subgroup $N=\left\langle R_{1}, R_{2}, \ldots\right\rangle$ inherits a metric from $G$. Let 
$g=\left(g_{n}\right)^{\omega} \in \operatorname{Con}^{\omega}(N, d)$. If $g_{n} \neq 1$, then $g_{n}=R_{1}^{\varepsilon_{1}} R_{2}^{\varepsilon_{2}} \cdots R_{j(n)}^{\varepsilon_{j(n)}}$, where $0 \leq \varepsilon_{i} \leq m-1$ for all $i$ and $\varepsilon_{j(n)} \neq 0$. Note that

$$
\operatorname{dist}\left(g_{n}, R_{j(n)}^{\varepsilon_{j(n)}}\right) \leq \sum_{i=1}^{j(n)-1}\left|R_{i}^{\varepsilon_{i}}\right| \leq(m-1) \sum_{i=1}^{j(n)-1}\left|R_{i}\right|=o_{\omega}\left(R_{j(n)}\right)
$$

by $\left(\mathbf{Q}_{3}\right)$, Lemma 4.13 (c), and Lemma 5.10. Hence

$$
\left(g_{n}\right)^{\omega}=\left(R_{i_{n}}^{s}\right)^{\omega}
$$

for some $s \in\{0,1, \ldots, m-1\}$. Now there are two cases to consider.

Case 1 Suppose that $\left(r_{n}\right)$ is not $(\omega, d)$-visible. Then (30) implies $\left(g_{n}\right)^{\omega}=(1)^{\omega}$, ie, $\operatorname{Con}^{\omega}(N, d)$ is a point. Applying the last assertion of Theorem 5.2 and Theorem 4.17, we obtain (b).

Case 2 Assume now that $\left(r_{n}\right)$ is $(\omega, d)$-visible (see Definition 4.16). Let $\left(R_{i_{n}}\right)$ be the sequence such that $r_{i_{n}}=\Theta_{\omega}\left(d_{n}\right)$. Note any sequence $\left(i_{n}^{\prime}\right)$ satisfying $r_{i_{n}^{\prime}}=\Theta_{\omega}\left(d_{n}\right)$ is $\omega$-equal to $\left(i_{n}\right)$ (that is, $i_{n}=i_{n}^{\prime} \omega$-almost surely) by $\left(Q_{4}\right)$ and Lemma 4.13 (c). Therefore $\operatorname{Con}^{\omega}(N, d)$ contains at most $m$ points.

In contrast, the points $\left(R_{i_{n}}^{s}\right)^{\omega}$ are different for different values of $s \in\{0,1, \ldots, m-1\}$. Indeed if $\left(R_{i_{n}}^{s}\right)^{\omega}=\left(R_{i_{n}}^{t}\right)^{\omega}$ for some $s \neq t, s, t \in\{0,1, \ldots, m-1\}$, then $\left|R_{i_{n}}^{s-t}\right|=$ $o_{\omega}\left(d_{n}\right)$. Passing from $s-t$ to $m-(s-t)$ if necessary, we obtain $\left|R_{i_{n}}^{l}\right|=o_{\omega}\left(d_{n}\right)=$ $o_{\omega}\left(r_{i_{n}}\right)$ for some $0<l \leq m / 2$. However this contradicts Lemma 5.10. Hence $\operatorname{Con}^{\omega}(N, d)$ consists of exactly $m$ points. Applying Theorem 5.2 again, we obtain that the map $\operatorname{Con}^{\omega}(G, d) \rightarrow \operatorname{Con}^{\omega}(G / N, d)$ induced by the natural homomorphism is locally isometric and each fiber consists of $m$ points, ie, $\operatorname{Con}^{\omega}(G, d)$ is an $m$-fold cover of $\operatorname{Con}^{\omega}(G / N, d)$. Note that by Theorem $4.17 \operatorname{Con}^{\omega}(G / N, d)$ is a circle-tree. Hence it contains cut points by Lemma 2.3. Therefore a finite subset $C \subset \operatorname{Con}^{\omega}(G, d)$ disconnects $\operatorname{Con}^{\omega}(G, d)$ if and only if $C$ contains a fiber of the map $\widehat{\alpha}$ : $\operatorname{Con}^{\omega}(G, d) \rightarrow$ $\operatorname{Con}^{\omega}(G / N, d)$ by Theorem 5.8. In particular, $c\left(\operatorname{Con}^{\omega}(G, d)\right)=m$.

Finally we note that $\left(Q_{4}\right)$ and Lemma 4.13 (c) guarantees that the second case occurs. The first case occurs when taking $d_{n}=r_{n}$ and any $\omega$.

Below we denote by $\mathbb{S}^{1}$ the unit circle with the lengths metric.

Theorem 5.12 There exists a finitely generated group $G$ and a scaling sequence $d=\left(d_{n}\right)$ such that for any ultrafilter $\omega, \operatorname{Con}^{\omega}(G, d)$ is bi-Lipschitz equivalent to the product of an $\mathbb{R}$-tree and $\mathbb{S}^{1}$. In particular, $\pi_{1}\left(\operatorname{Con}^{\omega}(G, d)\right)=\mathbb{Z}$. 
Proof Let $G(k)$ be the group corresponding to a sequence $k=\left(k_{n}\right)$ such that

$$
k_{n} \rightarrow \infty \text { and } k_{n} r_{n}=o\left(r_{n+1}\right) .
$$

The existence of such a sequence is guaranteed by the equality $r_{n}=o\left(r_{n+1}\right)$, which follows from $\left(Q_{4}\right)$ and Lemma 4.13 (c). Set $d_{n}=k_{n} r_{n}, d=\left(d_{n}\right)$. As in the proof of the previous theorem let $g=\left(g_{n}\right)^{\omega} \in \operatorname{Con}^{\omega}(N, d)$. If $g_{n} \neq 1$, then $g_{n}=R_{1}^{\varepsilon_{1}} R_{2}^{\varepsilon_{2}} \cdots R_{j(n)}^{\varepsilon_{j(n)}}$, where $0 \leq \varepsilon_{i} \leq k_{i}-1$ for all $i$ and $\varepsilon_{j(n)} \neq 0$. Note that

$$
\operatorname{dist}\left(g, R_{j(n)}^{\varepsilon_{j}(n)}\right) \leq \sum_{i=1}^{j(n)-1}\left|R_{i}^{\varepsilon_{i}}\right| \leq \sum_{i=1}^{j(n)-1} k_{i}\left|R_{i}\right|=o_{\omega}\left(R_{j(n)}\right)
$$

by (31) and Lemma 5.10. Therefore $\left(g_{n}\right)^{\omega}=\left(R_{j(n)}^{s(n)}\right)^{\omega}$ where

$$
s(n) \in\left\{0,1, \ldots, k_{n}-1\right\} .
$$

Note that if $\left(g_{n}\right)^{\omega} \neq(1)^{\omega}$, then $r_{j_{n}}=\Theta_{\omega}\left(\left|g_{n}\right|\right)=\Theta_{\omega}\left(d_{n}\right)$ and hence $j_{n}=n \omega$-almost surely. Thus $\left(g_{n}\right)^{\omega} \in \lim ^{\omega} p_{n}$, where $p_{n}$ is the cycle in $\Gamma(G,\{a, b\})$ that begins and ends at 1 and has label $R_{n}^{k_{n}}$. Since $\left|R_{n}\right|=r_{n}=o\left(d_{n}\right), \lim ^{\omega}\left(p_{n}\right)$ coincides with the set of points of type $\left(R_{n}^{s(n)}\right)^{\omega} \in \operatorname{Con}^{\omega}(N, d)$ and we obtain $\operatorname{Con}^{\omega}(N, d)=\lim ^{\omega} p_{n}$.

Observe that by Lemma $5.10 p_{n}$ is $(1 / 4,1)$-quasi-isometric to a circle of lengths $\left|p_{n}\right|=k_{n} r_{n}=d_{n}$. Hence $\lim ^{\omega} p_{n}$ is bi-Lipschitz equivalent to the unit circle. Note that by (31) the sequence $r_{n}$ is not $(\omega, d)$-visible. Hence $\operatorname{Con}^{\omega}(G / N, d)$ is an $\mathbb{R}$-tree by Theorem 4.17. Applying now Theorem 5.6, we obtain that $\operatorname{Con}^{\omega}(G, d)$ is bi-Lipschitz equivalent to the product of an $\mathbb{R}$-tree and $\mathbb{S}^{1}$.

Remark 5.13 Arguing as in the proof of Theorem 5.11 it is not hard to classify all asymptotic cones of the group $G(k)$ from the proof of Theorem 5.12 as follows. For any scaling sequence $d_{n}$ and any ultrafilter $\omega$, exactly one of the conditions (a)-(c) below holds and all possibilities can be realized.

(a) $\left(r_{n}\right)$ is $(\omega, d)$-visible, $\operatorname{Con}^{\omega}(G, d)$ is an infinite degree cover of a circle-tree.

(b) $\left(k_{n} r_{n}\right)$ is $(\omega, d)$-visible and $\operatorname{Con}^{\omega}(G, d)$ is bi-Lipschitz equivalent to the product of an $\mathbb{R}$-tree and a unit circle.

(c) There exists an $(\omega, d)$-visible sequence $\left(c_{n}\right)$ such that $c_{n}=o\left(k_{n} r_{n}\right)$ and $r_{n}=$ $o\left(c_{n}\right)$. In this case $\operatorname{Con}^{\omega}(G, d)$ is bi-Lipschitz equivalent to the product of an $\mathbb{R}$-tree and $\mathbb{R}$.

(d) There is no $(\omega, d)$-visible sequence $\left(c_{n}\right)$ such that $r_{n} \leq c_{n} \leq k_{n} r_{n}$. In this case $\operatorname{Con}^{\omega}(G, d)$ is an $\mathbb{R}$-tree.

In particular, even the finiteness of the connectedness number $c\left(\operatorname{Con}^{\omega}(G, d)\right)$ for a given group $G$ is not invariant under changing $d$ and $\omega$. 


\section{Lacunar hyperbolicity and divergence}

\subsection{Divergence of nonconstricted groups}

The following general statement allows one to estimate the divergence function of a group with no cut points in some of its asymptotic cones. Recall that given a path $p$ in a metric space, we denote by $p_{-}$and $p_{+}$the beginning and the ending points of $p$ respectively. The length of $p$ is denoted by $|p|$.

Theorem 6.1 Let $G$ be a finitely generated group. Suppose that for some sequence of scaling constants $d_{n}$ and every ultrafilter $\omega$, the asymptotic cone $\operatorname{Con}^{\omega}\left(G,\left(d_{n}\right)\right)$ does not have cut points. Let $f(n) \geq n$ be a nondecreasing function such that $d_{n} \leq f\left(d_{n-1}\right)$ for all sufficiently large $n$. Then the divergence function $\operatorname{Div}(n)$ of $G$ does not exceed $C f(n)$ for some constant $C$ (and all $n$ ).

Proof Since the asymptotic cone does not change if we change a finite subsequence of $\left(d_{n}\right)$, we can assume without loss of generality that $d_{1}=\frac{1}{4}$. Taking a constant multiple of $f$ if necessary, we can assume that $d_{n} \leq f\left(d_{n-1}\right)$ for all $n \geq 2$.

Since $\mathcal{C}$ does not have cut points for any choice of $\omega$, by [12, Theorem 2.1], we can conclude that there exists a constant $C_{1}$ such that $\operatorname{Div}(n)$ is bounded by $C_{1} n$ for every $n$ in any interval $\left[d_{k} / 18,18 d_{k}\right]$ for every $k \geq 2$.

Let $\delta=\frac{1}{4}, \lambda=2$. For every $n \geq 1$ choose elements $a_{n}, b_{n}, c_{n}$, in $G$ with $\operatorname{dist}\left(a_{n}, b_{n}\right) \leq n$ and such that $\operatorname{Div}_{\lambda}\left(a_{n}, b_{n}, c_{n} ; \delta\right)$ is maximal possible, ie

$$
\operatorname{Div}(n)=\operatorname{Div}_{\lambda}\left(a_{n}, b_{n}, c_{n} ; \delta\right) .
$$

Suppose, by contradiction, that $\operatorname{Div}(n)$ is not smaller than $C f(n)$ for some constant $C$ and all $n$. Then for every $m \geq 1$ there exists $n=n(m)$ such that $\operatorname{Div}(n)>m f(n)$. Pick $m>12+18 C_{1}$. Let $a=a_{n}, b=b_{n}, c=c_{n}$ where $n=n(m)$. Let $r=\operatorname{dist}(c,\{a, b\})$. Let $B$ be the ball of radius $\delta r$ around $c$. Without loss of generality assume that $\operatorname{dist}(c, a)=r$.

Note that any geodesic $h$ connecting $a$ and $b$ passes through $B$ since $\operatorname{Div}_{\gamma}(a, b, c ; \delta)>$ $n$. Hence $r \leq 2 n$. (Indeed, if $r>2 n$ every point in $h$ is at distance at least $r-|h| \geq$ $r-n>\frac{r}{2}>\delta r$ from $c$, and cannot belong to $B$.) Let $c^{\prime}$ be a point in $h \cap B$, so $c^{\prime} \in h$ is at distance at most $\delta r$ from $c$. Then $\operatorname{dist}\left(a, c^{\prime}\right) \leq r(1+\delta) \leq 2 r$.

Let $b^{\prime}$ be either $b$ or the point between $c^{\prime}$ and $b$ at distance $2 r$ from $c^{\prime}$. Let $h^{\prime}$ be the part of $h$ between $c^{\prime}$ and $b$. Then $h^{\prime}$ does not intersect $B$ (any point in $h^{\prime}$ is at distance $\geq(1-\delta) r>2 \delta r$ from $B)$. 
Since $\delta<\frac{1}{3}, \operatorname{dist}(a, B), \operatorname{dist}(b, B)>2 \delta r$ which exceeds the diameter of $B$.

Since the Cayley graph $\Gamma$ of $G$ is infinite, homogeneous and locally finite, for every vertex $x$ in $\Gamma$ there exists a bi-infinite geodesic $q(x)$ passing through $x$.

Consider the geodesic $q(a)$. The point $a$ cuts $q(a)$ into two geodesic rays $l(a)$ and $l(a)^{\prime}$. Since $\operatorname{dist}(a, B)$ is greater than the diameter of $B$, one of these rays does not pass through the ball $B$. Let it be $l(a)$. Similarly, let $l\left(b^{\prime}\right)$ be a geodesic ray starting at $b^{\prime}$ and not passing through $B$.

Choose the smallest $k \geq 2$ such that $r / 2 \leq d_{k}$. Note that then $d_{k-1} \leq r / 2$ (even if $k=2$ since $d_{1}=\frac{1}{4}$ and $\left.r \geq 1\right)$. Let $x$ be the point in $l(a)$ at distance $5 d_{k}$ from $a$, and let $y$ be the point in $l\left(b^{\prime}\right)$ at distance $5 d_{k}$ from $b^{\prime}$.

Case 1 Suppose that $\operatorname{dist}(x, y)<d_{k} / 2$. Then consider a geodesic $p$ connecting $x$ and $y$. Any point in $p$ is at distance at least $5 d_{k}-d_{k} / 2>2 r$ from $a$. Hence any point in $p$ is at distance at least $2 r-r>\delta r$ from $c$. Thus $p$ does not intersect $B$, so we found a path $[a, x] \cup p \cup[y, b] \cup h^{\prime}$ of length at most $11 d_{k}+n$ connecting $a$ and $b$. Then

$$
11 d_{k}+n \geq \operatorname{Div}_{\lambda}(a, b, c ; \delta)=\operatorname{Div}(n) \geq m f(n) .
$$

But $11 d_{k}+n \leq 11 f\left(d_{k-1}\right)+n \leq 11 f(r / 2)+n \leq 12 f(n)$ since $f$ is a nondecreasing function and $f(n) \geq n$. Thus $12 f(n) \geq m f(n)$, a contradiction since $m>12$.

Case 2 Suppose that $\operatorname{dist}(x, y) \geq d_{k} / 2$. Since $\operatorname{dist}(x, y) \leq 10 d_{k}+\operatorname{dist}\left(a, b^{\prime}\right) \leq$ $10 d_{k}+4 r \leq 18 d_{k}$, the distance $\operatorname{dist}(x, y)$ is in the interval $\left[d_{k} / 18,18 d_{k}\right]$, and so there exists a path $p$ of length at most $C_{1} \operatorname{dist}(x, y)$ avoiding the ball of radius $\delta \operatorname{dist}(c,\{x, y\})>\delta\left(5 d_{k}-r\right) \geq \delta\left(5 d_{k}-2 d_{k}\right)>\delta r$ around $c$. Then the path $[a, x] \cup p \cup\left[y, b^{\prime}\right] \cup h^{\prime}$ connects $a$ and $b$, avoids $B$, and has length at most

$$
10 d_{k}+C_{1}\left(18 d_{k}\right)+n \leq\left(10+18 C_{1}\right) f\left(d_{k-1}\right)+f(n) \leq\left(11+18 C_{1}\right) f(n),
$$

a contradiction since $m>11+18 C_{1}$.

\subsection{Torsion groups with slow nonlinear divergence}

Let $F_{2}=\langle a, b\rangle$ be the free group of rank 2. We fix an arbitrary odd prime $p$ and a large odd power $n_{0}$ of $p$, say, $n_{0}>10^{80}$. Let $G(0)=F_{2}$, ie the set of relators $\mathcal{R}_{0}$ of rank 0 is empty. The set of periods of rank 0 is empty by definition. Below we define the sets $\mathcal{R}_{i}$ of defining relations of groups $G(i)$ and an increasing sequence $d=\left(d_{r}\right)$ by induction. This sequence depends on a nondecreasing function $\phi$ such that $\phi(0)=0, \phi(1)=1, \phi(r) \geq 2$ for every $r=2,3 \ldots$, and $\lim _{r \rightarrow \infty} \phi(r)=\infty$. 
Two arbitrary segments from the set $\left\{\left(d_{r} / \phi(r), \phi(r) d_{r}\right] \mid r=1,2, \ldots\right\}$ will have empty intersection, and $d_{0}=1$. After $d_{r-1}$ is defined we introduce $d_{r}$ and then define all groups $G(i)$ for $\phi(r-1) d_{r-1}<i \leq \phi(r) d_{r}$.

Assume $r>0$ and $d_{0}, \ldots d_{r-1}$ are already defined along with hyperbolic groups

$$
G(0), G(1), \ldots, G\left(i_{r-1}\right)
$$

where $i_{r-1}=\left[\phi(r-1) d_{r-1}\right]$. Let $\delta_{i_{r-1}}$ be the hyperbolicity constant of $G_{i_{r-1}}$. We chose a minimal integer $d_{r}$ such that

$$
d_{r} \geq \max \left\{\phi(r)^{2} d_{r-1}, \phi(r)^{2} \delta_{i_{r-1}}, 2\right\} .
$$

For example, $d_{1}=2$. We also define $i_{r}=\left[\phi(r) d_{r}\right]$.

Then we argue by induction on $i\left(i_{r-1}<i \leq i_{r}\right)$. A word $A$ is called simple in rank $i-1$ if it is not conjugate in rank $i-1$ (that is in $G(i-1)$ ) to $B^{m}$, where $|B|<|A|$ or $B$ is a period of rank $j \leq i-1$.

Let $\mathcal{X}_{i}$ be a maximal set of simple in rank $i-1$ words of length $i$ such that for two different $A, B \in \mathcal{X}_{i}$, we have that $A$ is not conjugate of $B^{ \pm 1}$ in rank $i-1$. All the words from $\mathcal{X}_{i}$ are called periods of rank $i$.

For every period $A$ of rank $i\left(i_{r-1}<i \leq i_{r}\right)$, we introduce a large odd exponent $n_{A}$, where $n_{A}$ is a minimal power of $p$ such that

$$
\begin{array}{ll}
n_{A} \geq \max \left(n_{0}, d_{r} / i\right) & \text { for } i_{r-1}<i<d_{r} / \phi(r), \\
n_{A}=n_{0} & \text { for } d_{r} / \phi(r) \leq i \leq i_{r} .
\end{array}
$$

The set $\mathcal{R}_{i}$ is, by definition, equal to $\mathcal{R}_{i-1} \cup\left\{A^{n_{A}} \mid A \in \mathcal{X}_{i}\right\}$, and $G(i)=\left\langle a, b \mid \mathcal{R}_{i}\right\rangle$. We will show in Lemma 6.6 that the group $G(i)$ is hyperbolic. Finally,

$$
G=G\left(n_{0}, \phi\right)=\left\langle a, b \mid \bigcup_{i=0}^{\infty} \mathcal{R}_{i}\right\rangle
$$

Since $d_{0}=1$ and $d_{1}=2$ we can chose $\mathcal{R}_{1}=\left\{a^{n_{0}}, b^{n_{0}}\right\}$, and $G(1)$ is the free product of two cyclic groups of order $n_{0}$. Since $i_{1} \geq d_{1}=2$, one can set $\mathcal{R}_{2}=\left\{a, b, a b, a b^{-1}\right\}$. Thus $a$ and $b$ are periods of rank 1 , and $a b$ and $a b^{-1}$ are periods of rank 2 . Hence, for every word $w$ of length at most 2 , we have $w^{n_{0}}=1$ in $G$.

The proof of the following Proposition is based on [41] and is contained in the next section. 
Proposition 6.2 The 2-generated group $G$ satisfies the following properties:

(a) The natural homomorphism $G\left(i_{r-1}\right) \rightarrow G\left(i_{r}\right)$ is injective on the ball of radius $K d_{r} / \phi(r)$, for a nonzero constant $K$.

(b) (Bounded torsion up to a small deformation) There is a constant $c>1$ such that for every large enough integer $r$ and every word $W$ with $c d_{r} / \phi(r)<|W|_{G}<$ $d_{r} \phi(r) / c$, there exists a word $U$ of length $\leq 1$ such that $(W U)^{n_{0}}=1$ in $G$.

(c) (Relations are locally quasigeodesic) For every large enough $r$ there exists a relation $u_{r}^{n_{0}}=1$ in $\bigcup_{i=0}^{\infty} \mathcal{R}_{i}$ with $\left|u_{r}\right|=d_{r}$, such that no nonempty subword $w$ of $u_{r}^{n_{0}}$ of length $<n_{0}\left|u_{r}\right| / 2$ can be equal to a word of length at most $|w| / 2$ in $G$.

(d) (Finite cyclic centralisers) $G$ is an infinite $p$-group in which the centralizer of every nontrivial element is cyclic.

(e) The hyperbolic constant $\delta_{i_{r}}$ of the group $G\left(i_{r}\right)$ is $O\left(\phi(r) d_{r}\right)$.

(f) The order of arbitrary word $X$ in the generators of $G$ is $O\left(\phi(|X|)^{3}\right)$.

Theorem 6.3 Let $G$ and $d=\left(d_{n}\right)$ be the group and the scaling sequence constructed above. Then $G$ is lacunary hyperbolic, but for any nonprincipal ultrafilter $\omega$, the asymptotic cone $\mathfrak{C}=\operatorname{Con}^{\omega}(G, d)$ does not have cut points.

First we note that by (32) we have $\delta_{r-1}=o\left(d_{r} / \phi(r)\right)$. Together with the first assertion of Proposition 6.2 and Theorem 3.3 this implies the first assertion of the Theorem.

Let us now prove that $\mathfrak{C}$ does not have cut points. Indeed suppose this is not so. Then $\mathfrak{C}$ is a tree-graded space with respect to the collection $\mathcal{C}_{1}$ of maximal subsets without cut points (see Lemma 2.2). Let $u_{r}, r=1,2, \ldots$ be the words given by Proposition 6.2 (c). Then the limit of the loops $p_{r}$ in the Cayley graph of $\mathcal{G}$ corresponding to the relations $u_{r}^{n_{0}}=1$ is a nontrivial loop in $\mathfrak{C}$ (of length $n_{0}$ ). Indeed, if $\lim ^{\omega} p_{r}$ is not a simple loop then $\omega$-a.s. there are two points $x_{r}$ and $y_{r}$ on $p_{r}$ that are distance $\Theta\left(d_{r}\right)$ apart along the loop $p_{r}$ but $o\left(d_{r}\right)$-close in the Cayley graph of $G$. But this would contradict Proposition 6.2 (c). Therefore $\mathfrak{C}$ is not an $\mathbb{R}$-tree.

Hence some of the pieces in $\mathfrak{C}$ contain infinitely many points.

Since $\mathfrak{C}$ is homogeneous, one of these pieces, $M$, contains $O=(1)^{\omega}$. Let $A$ be another point in $M$.

As in the proof of [13, Lemma 6.10] consider two cases.

Case 1 Suppose that there are two pieces from $\mathcal{C}_{1}$ that intersect. Then every point is in two distinct pieces. Then we can construct a geodesic $g:[0, s] \rightarrow \mathfrak{C}_{1}$ such that 
$s=\sum_{i=1}^{\infty} s_{i}$ with $0<s_{i}<1 / i^{2}$ and $g\left[\sum_{i=0}^{r} s_{i}, \sum_{i=0}^{r+1} s_{i}\right] \subset M_{r}$ for some pieces $M_{r}$, where $M_{r} \neq M_{r+1}$ for all $r \in \mathbb{N} \cup\{0\}$. Here $s_{0}=0$. Such a geodesic exists by Lemma 2.4. We call such a geodesic fractal at the arrival point. That geodesic with reverse orientation will be called fractal at the departure point. If $g$ is fractal at the departure point, $g^{\prime}$ is fractal at the arrival point, $g_{+}=g_{-}^{\prime}$, we can construct (using Lemma 2.4) a geodesic $p$ which is a composition of an initial piece of $g$ and the terminal piece of $g^{\prime}$. The geodesic $p$ is then fractal at the departure and arrival points or bifractal. By homogeneity, every point in $\mathfrak{C}$ is the endpoint of a bifractal geodesic.

Let $[A, B]$ be a bifractal geodesic. Lemma 2.5, part (2), implies that $[A, B]$ can intersect a piece $M$ containing $A$ in $A$ or in a nontrivial subgeodesic $\left[A, B^{\prime}\right]$. Since $[A, B]$ is fractal at the departure point the latter case cannot occur. It follows that the intersection of $[A, B]$ and $M$ is $\{A\}$. There exists an isometry $\gamma=\left(x_{r}\right) \in G_{e}^{\omega}(d)$ such that $\gamma(O)=B$. Since $[A, B]$ is fractal at the arrival point also, it follows that $[A, B] \cap \gamma M=\{B\}$. For every $Z \in \gamma M$ we have that $[A, B] \cup[B, Z]$ is a geodesic, by Lemma 2.4. In particular $A$ is the projection of $\gamma M$ onto $M$. A symmetric argument gives that $B=\gamma(O)$ is the projection of $M$ onto $\gamma M$.

Note that in the argument of the previous paragraph we only used the fact that $\gamma O=B$. Let us change $\gamma$ a little bit preserving the property. Then the conclusions of the previous paragraph will still be true.

Let $w_{r}$ be the shortest word representing $x_{r}^{-1}$ in $G$. Note that $\frac{1}{C} d_{r} \leq\left|w_{r}\right| \leq C d_{r}$ for $\omega$-almost every $r$ where $C$ is a constant. By Proposition 6.2 (b), $\omega$-a.s. there exist words $u_{r}$ with $\left|u_{r}\right| \leq 1$ such that $\left(w_{r} u_{r}\right)^{n_{0}}=1$ is in $\mathcal{R}$. Let $\beta=\left(y_{r} x_{r}\right) \in G_{e}^{\omega}(d)$ where $y_{r} \in G$ is represented by $u_{r}^{-1}$. Then again $\beta(O)=B$. Notice that $\beta^{n_{0}}=1$.

For every $k \geq 2$ consider the piecewise geodesic path from $\beta^{k} O \in \beta^{k} M$ to $A$

$$
g=\left[\beta^{k} O, \beta^{k-1} A\right] \cup\left[\beta^{k-1} A, \beta^{k-1} O\right] \cup\left[\beta^{k-1} O, \beta^{k-2} A\right] \cup \cdots \cup[\beta O, A]
$$

where every odd numbered segment is a bifractal geodesic and every even segment is a nontrivial geodesic inside a piece. By Lemma 2.4, $g$ is a geodesic which is not inside $M$. By the strong convexity of pieces in a tree-graded space (Lemma 2.5), we conclude that $\beta^{k} O \notin M$, so $\beta^{k} M \neq M$. This contradicts the equality $\beta^{n_{0}}=1$.

Case 2 Now suppose that all pieces in $\mathcal{C}_{1}$ are disjoint. Note that we could repeat the argument from Step 1 if we found an isometry $\delta$ from $G_{e}^{\omega}(d)$ such that $B=\delta(O) \notin M$, the projection of $M$ onto $\delta(M)$ is $B$ and the projection of $\delta(M)$ onto $M$ is $A$. Indeed, by slightly changing $\delta$, we can find an isometry $\beta$ with the same property and, in addition, $\beta^{n_{0}}=1$. On the other hand, for every $k$, consider the piecewise geodesic curve (33). Every even numbered geodesic segment $g_{i}$ in it is nontrivial and inside 
a piece $M_{i}$, and every odd numbered geodesic segment connects a point $U_{i}$ in a piece $M_{i}$ and a point $U_{i+1}$ in a piece $M_{i+1}$ such that $U_{i}$ is the projection of $M_{i+1}$ onto $M_{i}$ and $U_{i+1}$ is a projection of $M_{i}$ onto $M_{i+1}$. Hence by Lemma 2.6, the odd numbered geodesic segments $g_{i}$ intersect $M_{i}$ (resp. $M_{i+1}$ ) in exactly one point. By Lemma 2.4 the curve (33) is a geodesic, and so $\beta^{k}(O) \neq O$ for any $k$, a contradiction.

Thus our goal is to find such $\delta$.

Lemma 2.2, part (b), implies that $A$ is the projection of a point $B \in \mathfrak{C} \backslash M$. Let $\gamma=\left(x_{r}\right)$ be an isometry from $G_{e}^{\omega}$ such that $\gamma(O)=B=\left[x_{r}^{-1}\right]$. If $[A, B]$ intersects $\gamma(M)$ in $B$ then we have found the desired $\delta=\gamma$ by Lemma 2.6 (since the isometries of $\mathfrak{C}$ permute the pieces of $\left.\mathcal{C}_{1}, \gamma(M) \in \mathcal{C}_{1}\right)$.

Assume $[A, B] \cap \gamma(M)=\left[B^{\prime}, B\right], B^{\prime} \neq B$ Since all the pieces are disjoint, $B^{\prime} \neq A$. We have $B^{\prime}=\gamma\left(A^{\prime}\right)$ for some $A^{\prime} \in M$. Since the space $\mathfrak{C}$ is homogeneous, and all pieces of $\mathcal{C}_{1}$ are disjoint, the stabilizer of $\gamma(M)$ in $G_{e}^{\omega}(d)$ acts transitively on $\gamma(M)$. Then there exists $\gamma^{\prime}$ in it such that $\gamma^{\prime}(B)=B^{\prime}$. We have that $\gamma^{\prime} \gamma(M)=\gamma(M)$ projects onto $M$ in $A$ and $M$ projects onto $\gamma^{\prime} \gamma(M)$ in $B^{\prime}=\gamma^{\prime} \gamma(O)$, so we can take $\delta=\gamma^{\prime} \gamma$

Proposition 6.2 and Theorem 6.3 imply that the divergence and the orders of elements of $G\left(n_{0}, \phi\right)$ can grow arbitrarily slow.

Corollary 6.4 For any positive function $f$ with $f(r) / r$ nondecreasing satisfying $\lim _{r \rightarrow \infty} f(r) / r=\infty$, and for any nondecreasing function $g(r) \lim _{r \rightarrow \infty} g(r)=\infty$, there is a function $\phi$ such that

(a) for some $\lambda$, the divergence function $\operatorname{Div}(r, \lambda)$ of the group $G=G\left(n_{0}, \phi\right)$ is $O(f(r))$, is not linear, but does not exceed a linear function on an infinite subset of $\mathbb{N}$;

(b) the order of any element $x \in G\left(n_{0}, \phi\right)$ is $O(g(|x|))$.

Proof Consider the functions $f$ and $g$ from the formulation of the corollary. Then we can choose a nondecreasing function $\phi$ such that $\lim _{r \rightarrow \infty} \phi(r)=\infty, \phi(r) \geq 2$ for any $r \geq 2$, and $\phi(r)^{3}<\min (c f(r-1) /(r-1), g(r))$ for every $r \geq 2$ and a constant $c$. Then, by (32) and Condition (e) of Proposition 6.2, we have $d_{r} \leq c^{\prime} \phi(r)^{3} d_{r-1}$ for some constant $c^{\prime}$ and every $r>0$. The right-hand side is less than $c^{\prime} c f(r-1) d_{r-1} /(r-1) \leq$ $C f\left(d_{r-1}\right)$ for $C \geq c^{\prime} c$ since the function $f(r) / r$ is nondecreasing and $d_{r-1} \geq r-1$ by (32), if $r \geq 2$. Besides, $d_{1}<C f\left(d_{0}\right)$ if $C$ is large enough. Now the statement on the divergency follows from Theorem 6.1. Condition (f) obviously implies the second statement of the corollary. 


\subsection{The proof of Proposition 6.2}

Here we present the proof of Proposition 6.2. We can apply lemmas from [41] to the construction of group $G$ from that proposition because it obviously satisfies the $R$-condition from [41, Section 25] since we do not use relations of the "second type" here. In particular, every reduced diagram of rank $i$ arising below is a B-map by [41, Lemma 26.5]. (See the definition in [41, Subsection 20.4].) The contiguity diagrams we use now are more particular than those in the previous sections. (See their definition in Subsection 20.1 of [41]).

Notation 6.5 As in [41, Chapter 7] we fix certain positive numbers $\eta \ll \zeta \ll \epsilon \ll \gamma \ll$ $\beta \ll \alpha$ between 0 and 1 where $\ll$ means "much smaller". Here "much" means enough to satisfy all the inequalities in Chapters 7 and 8 of [41]. We also have $n_{0}^{-1} \ll \eta$.

Denote by $P(i)$ the maximum of $n_{A}|A|$ for the periods $A$ of rank at most $i$, that is the maximum length of relations of rank at most $i$.

Lemma 6.6 The group $G(i)$ is $\delta_{i}$-hyperbolic for arbitrary $i \leq i_{r}=\left[\phi(r) d_{r}\right]$, where $\delta_{i}=n_{0} P(i)$.

Proof Step 1 First we want to prove that a geodesic subpath $p$ of a boundary $\partial \Delta$, where $\Delta$ is a reduced diagram of rank $i$, is a smooth section of rank $k$ (see the formulations of the smoothness conditions $S 1-S 5$ in Subsection 20.4 of [41]) if we define $\operatorname{rank}(p)=k=\left[(1-2 \beta)^{-1} P(i)+1\right]$.

Condition $S 1$ holds since $p$ is geodesic.

Assume that $\Gamma$ is a contiguity diagram of a cell $\Pi$ to a geodesic subpath $p$ in a reduced diagram $\Delta$ over $G(i)$ with $(\Pi, \Gamma, p) \geq \varepsilon$. Let $\partial(\Pi, \Gamma, p)=p_{1} q_{1} p_{2} q_{2}$. Then by [41, Lemma 21.2], $\left|q_{1}\right|>(1-2 \beta)\left|q_{2}\right|$. Indeed, the proof of [41, Lemma 21.2] does not change if one replaces "smooth $p$ " by "geodesic $p$ ".

Let $A^{n_{A}}$ be the label of $\partial \Pi$. Since $\left|q_{1}\right| \leq P(i)$, we have $\left|q_{2}\right|<(1-2 \beta)^{-1} P(i)$. This implies condition $S 2$ from [41, Section 20.4] for $p$ since then $\left|q_{2}\right|<(1+\gamma) k$. Since our relations are of the first type (ie, of the form $A_{A}^{n}$, and so the boundary of any cell is just one "long section" [41, Section 25]), $S 2$ implies $S 3$, and $S 4$ is obvious because $1<\alpha^{-1}$. Condition $S 5$ automatically holds since $k>i$, and therefore there are no cells of rank $k$ in $\Delta$. We conclude that $p$ is a smooth section of rank $k$ in $\partial \Delta$.

Step 2 Now let $x y z$ be a triangle in the Cayley graph of $G(i)$ with geodesic paths $x$, $y$, and $z$. To prove that $x$ belongs to $n_{0} P(i)$-neighborhood of $y \cup z$, we introduce an inscribed geodesic hexagon $\Psi=t_{1} x^{\prime} t_{2} y^{\prime} t_{3} z^{\prime}$, where (1) $x^{\prime}, y^{\prime}$, and $z^{\prime}$ are subpaths of 
$x, y$, and $z$, respectively, (2) $\max \left(\left|t_{1}\right|,\left|t_{2}\right|,\left|t_{3}\right|\right) \leq \eta^{-1} k$, and the sum $\left|x^{\prime}\right|+\left|y^{\prime}\right|+\left|z^{\prime}\right|$ is minimal for hexagons satisfying (1) and (2). (It follows that if $\left|x^{\prime}\right|>0$, then the distance between any point of $x^{\prime}$ and $y^{\prime} \cup z^{\prime}$ is at least $\eta^{-1} k$. Similar properties hold for $y^{\prime}$ and $z^{\prime}$.)

Then we have decompositions $x=x_{1} x^{\prime} x_{2}, y=y_{1} y^{\prime} y_{2}$, and $z_{1} z^{\prime} z_{2}$. If $\left|x_{1}\right|<$ $2 \gamma^{-1} \eta^{-1} k$, then every point $o$ of $x_{1}$ is at the distance at most $2 \gamma^{-1} \eta^{-1} k$ from $z_{2}$. If $\left|x_{1}\right| \geq 2 \gamma^{-1} \eta^{-1} k \geq 2 \gamma^{-1}\left|t_{1}\right|$, then we can apply [41, Lemma 22.4] to a reduced diagram with boundary $x_{1} t_{1}^{-1} z_{2}$ where, according to Step 1 , sections $x_{1}$ and $z_{2}$ are smooth of rank $k$. By [41, Lemma 22.4], again, every point $o$ of $x_{1}$ can be connected to a point of $z_{2}$ by a path of length $<2 \gamma^{-1} \eta^{-1} k<3 \gamma^{-1} \eta^{-1} P(i)<\frac{1}{2} n_{0} P(i)$.

Similarly, the distance from every point of $x_{2}$ to $y_{1}$ is less than $\frac{1}{2} n_{0} P(i)$. Thus to complete the proof, it suffices to show that $\left|x^{\prime}\right|<n_{0} P(i)$. Proving by contradiction, we suppose $\left|x^{\prime}\right| \geq n_{0} P(i)>\frac{1}{2} n_{0} k$ and consider a reduced diagram $\Delta$ of rank $i$ with boundary $z^{\prime} t_{1} x^{\prime} t_{2} y^{\prime} t_{3}$. First let us check that $\Delta$ is a $C$-map in the meaning of [41, Section 23.1] with 3 long sections of the first type having rank $k$, namely, $s_{0}=z^{\prime}$, $s_{1}=x^{\prime}, s_{2}=y^{\prime}$, with short sections $t_{1}, t_{2}, t_{3}$, and with paths $p_{1}, p_{2}, q$ of zero length (in the notation of [41]).

Since $\left|s_{1}\right|=\left|x^{\prime}\right| \geq \frac{1}{2} n_{0} k$, the diagram $\Delta$ satisfies condition $C 1$ and $C 2$ with $j=k$ because $l=3-1=2$ for 3 sections $s_{1}, s_{2}, s_{3}$. Conditions $C 3$ and $C 4$ hold since $|q|=\left|p_{1}\right|=\left|p_{2}\right|=0$ and $s_{1}, s_{2}, s_{3}$ are geodesic in $\Delta$. Condition $C 5$ holds since $\max \left\{\left|t_{1}\right|,\left|t_{2}\right|,\left|t_{3}\right|\right\}<\eta^{-1} k$ (and $\eta^{-1}=d$ in the book [41]). It follows from the choice of $\Psi$ that there are no contiguity subdiagrams between either $x^{\prime}$ and $y^{\prime}$ or $x^{\prime}$ and $z^{\prime}$, or $y^{\prime}$ and $z^{\prime}$, since, by [41, Lemma 21.1(1)], the side arcs of such a subdiagram would be of length $\left\langle\eta^{-1} k\right.$. Therefore $\Delta$ satisfy condition $C 6$. The condition $C 7$ holds for the same reason as $S 4$ at Step 1. Thus $\Delta$ is a $C$-map.

Since $\Delta$ is a $C$-map, there must be a contiguity subdiagram between a pair of sides from $\left\{x^{\prime}, y^{\prime}, z^{\prime}\right\}$ by [41, Lemma 23.15]. But this is impossible as was shown in the previous paragraph, which gives a contradiction.

Lemma 6.7 The natural homomorphism $G\left(i_{r-1}\right) \rightarrow G\left(i_{r}\right)$ is injective on the ball of radius $0.4 d_{r} / \phi(r)$.

Proof Let $w$ be a word equal to 1 in $G\left(i_{r}\right)$ but not in $G\left(i_{r-1}\right)$. Then there is a reduced diagram $\Delta$ such that its boundary label is $w$ and it contains a cell $\Pi$ of a rank $j>i_{r-1}$. It follows from the construction of defining words of rank $j>i_{r-1}$ that the perimeter $|\partial \Pi|$ of $\Pi$ is at least $d_{r} / \phi(r)$. Therefore, by Lemma 23.16 of [41],

$$
|w|=|\partial \Delta|>(1-\alpha)|\partial \Pi| \geq(1-\alpha) d_{r} / \phi(r)>0.8 d_{r} / \phi(r) .
$$

The inequality $|w|>0.8 d_{r} / \phi(r)$ gives the injectivity radius at least $0.4 d_{r} / \phi(r)$. 
The following lemma seems to be known. The short proof of it has been communicated to the authors by Ian Agol.

Lemma 6.8 Let $o_{1}$ and $o_{2}$ be two distinct points on the boundary of a double punctured disk $D$, and $x$ the boundary cycle starting (and ending) at $o_{1}$. Let $y$ be a simple path connecting $o_{1}$ and $o_{2}$ in $D$ and separating the punctures, and $z$ a simple loop starting at $o_{1}$ and going around exactly one of the punctures. Then every simple path connecting $o_{1}$ and $o_{2}$ in $D$ is homotopic to $x^{s} p_{1} x^{-s} p_{2}$ where each of $p_{1}, p_{2}$ is a product of at most 2 factors from $\left\{x, y, z^{ \pm 1}\right\}$.

Proof Let $\mathcal{D}$ be the diffeomorphism group of $D$. (The elements of $\mathcal{D}$ leave the boundary $\partial D$ invariant and may permute the punctures.) Denote by $\mathcal{D}_{0}$ the subgroup of $\mathcal{D}$ that fixes every point on the boundary of the disc $\partial D$. It is well known that, modulo diffeomorphisms isotopic to the identity element, $\mathcal{D}_{0}$ is the cyclic braid group $B_{2}=\langle\sigma\rangle$.

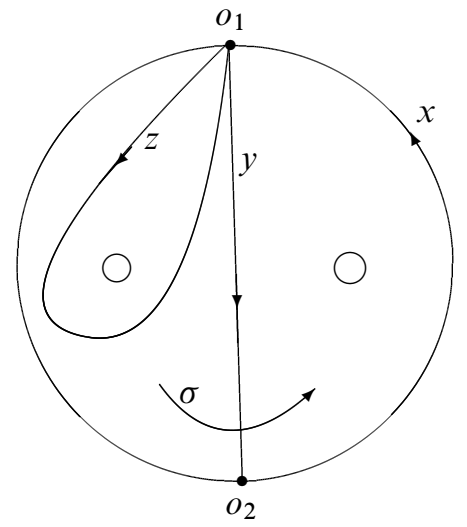

Figure 7: The paths $x, y, z$ and the diffeomorphism $\sigma$

Up to the action of $\mathcal{D}$, there are only two arcs with boundary on the disk: one inessential isotopic into the boundary, and one essential separating the two punctures. It is not hard to see that any essential arc connecting $o_{1}$ and $o_{2}$ is the image of $y$ under some diffeomorphism from $\mathcal{D}_{0}$.

One computes that: $\sigma^{2 k}(z)=x^{-k} z x^{k}, \sigma^{2 k}(x)=x$ (which is the well-known action of $B_{2}$ on the fundamental group of the punctured disk). There are two boundary parallel arcs, $z y$ and $x z y$, both of which are fixed by $\sigma$. We may also compute that $\sigma(y)=z x z y$. 
Now, we compute that

$$
\sigma^{2 k}(y)=\sigma^{2 k}\left(z^{-1} z y\right)=\sigma^{2 k}\left(z^{-1}\right) z y=x^{-k} z^{-1} x^{k} z y .
$$

We may also compute

$$
\sigma^{2 k+1}(y)=\sigma^{2 k}(z x z y)=\sigma^{2 k}(z) x z y=x^{-k} z x^{k} x z y=x^{-k-1} x z x^{k+1} z y .
$$

In each case, we see that the arcs are in the normal form $x^{k} p_{1} x^{-k} p_{2}$, where each of $p_{1}, p_{2}$ is a product of at most 2 factors from $\left\{x, y, z^{ \pm 1}\right\}$.

Lemma 6.9 Let $W$ be a word with $|W|_{G}>12 n_{0}$. Then for at most one word $U$ of length $\leq 1$, the word $W U$ is conjugate in $G$ to a word of length $\leq|W|_{G} / 15$.

Proof By contradiction, assume that there are two distinct words $U_{1}$ and $U_{2}$ of length at most 1 such that $W U_{1}$ and $W U_{2}$ are conjugate in $G$ to some $V_{1}$ and $V_{2}$, respectively, with $\left|V_{1}\right|,\left|V_{2}\right| \leq|W|_{G} / 15$. Then we have two annular diagrams $\Delta_{k}$ $(k=1,2)$ over $G$ with contours $w_{k} u_{k}$ and $v_{k}$ such that $\operatorname{Lab}\left(w_{k}\right) \equiv W, \operatorname{Lab}\left(u_{k}\right) \equiv U_{k}$, and $\operatorname{Lab}\left(v_{k}\right) \equiv V_{k}$.

Since $\operatorname{Lab}\left(w_{1}\right) \equiv \operatorname{Lab}\left(w_{2}\right)$ we can identify the paths $w_{1}$ and $w_{2}$ and obtain a diagram $\Gamma$ on a disk with two holes. The holes are bounded by paths $v_{1}$ and $v_{2}^{-1}$, and the third boundary component of $\Gamma$ is $x=u_{1} u_{2}^{-1}$. There are two vertices $o_{1}$ and $o_{2}$ on the boundary of $\Gamma$ connected by three paths, namely, by $u_{1}^{-1}$, by $u_{2}$, and by $w=w_{1}=w_{2}$. Let $\Delta$ be a reduced diagram with the same boundaries obtained from $\Gamma$.

Note that the words $W U_{k}$ are not nontrivial in $G$ since $|W|_{G}>\left|U_{k}\right|_{G}$. So are the words $V_{1}$ and $V_{2}$. The word $X \equiv U_{1} U_{2}^{-1}$ is also nontrivial since every nontrivial in $F_{2}$ word of length at most 2 is also nontrivial in $G$ by [41, Lemma 23.16]. Therefore [41, Lemma 22.2] is applicable to $\Delta$, and there is a simple path $t$ in $\Delta$ connecting some vertices $O_{1}$ on $x$ and $O_{2}$ on $v_{1}$ such that $|t|<(1 / 2+4 \gamma)\left(\left|v_{1}\right|+\left|v_{2}\right|+|x|\right)$. Hence $o_{1}$ is connected with $O_{2}$ by a simple path $s$ of length $|s|<(1 / 2+4 \gamma)\left(2|W|_{G} / 15+2\right)+1$ because $\left|V_{k}\right| \leq|W|_{G} / 15$ and $\left|U_{k}\right| \leq 1$. Therefore there is a simple loop $z$ starting at $o_{1}$ and surrounding the hole bounded by $v_{1}$ such that

$$
|z| \leq 2|s|+\left|v_{1}\right|<(1+8 \gamma)\left(2|W|_{G} / 15+2\right)+2+|W|_{G} / 15<0.205|W|_{G}
$$

since $|W|_{G}>12 n_{0}$ and $\gamma$ is very small.

On the one hand, vertices $o_{1}$ and $o_{2}$ are connected by the simple path $w$ labeled by $W$ in $\Gamma$. The reduction process (the cancellations of cells) preserves these properties of $w$ in the following sense (see [41, Section 13]): There is a simple path $w^{\prime}$ in $\Delta$ connecting $o_{1}$ and $o_{2}$ such that its label $W^{\prime}$ is equal to $W$ in $G$. 
On the other hand, $y=u_{1}^{-1}$ also connects $o_{1}$ and $o_{2}$ in $\Delta$, and $|y| \leq 1$. The path $w^{\prime}$ is homotopic for some $e$, to $p_{1} x^{e} p_{2} x^{-e}$ where $|p|_{1},\left|p_{2}\right|<2 \times 0.205|W|_{G}=0.41|W|_{G}$, by Lemma 6.8 .

Hence $|W|_{G}=\left|W^{\prime}\right|_{G} \leq\left|p_{1}\right|+\left|p_{2}\right|+2\left|x^{e}\right|_{G}<0.82|W|_{G}+2\left|X^{e}\right|_{G}$. But $\left|X^{e}\right|_{G} \leq n_{0}$ since we have $X^{n_{0}}=1$ in $G$ for every word $X$ of length at most 2. Therefore we obtain $0.18|W|_{G} \leq 2 n_{0}$ against the assumption of the lemma. The lemma is proved.

The following lemma gives Condition (b) of Proposition 6.2:

Lemma 6.10 There is a constant $c>1$ such that for every integer $r \geq 1$ and every word $W$ with $\max \left(12 n_{0}, c d_{r} / \phi(r)\right)<|W|_{G}<d_{r} \phi(r) / c$, there exists a word $U$ of length $\leq 1$ such that $(W U)^{n_{0}}=1$ in $G$.

Proof We set $c=150 \zeta^{-1}$ where $\zeta$ is the small positive number from Chapter 7 of the book [41]. Then we may assume that $W$ is a geodesic word in $G$, that is $|W|=|W|_{G}$.

By Lemma 6.9, there exist four different words $U_{k}(1 \leq k \leq 4)$ of length at most 1 such that $W U_{k}$ is not conjugate in $G$ to a word of length $\leq|W| / 15$. On the other hand, since $|W U|<2|W|$, it follows from the definition of $G(i)$ that $W U_{k}$ must be conjugate in rank $i=2|W|$ to a power $A_{k}^{m_{k}}$ where $A_{k}$ is either period of some rank $j_{k} \leq i$ or a simple in rank $i$ word. Respectively, we have four reduced annular diagrams $\Delta_{k}$ of rank $i$ with boundary paths $w_{k} u_{k}$ and $p_{k}$ where $\operatorname{Lab}\left(w_{k}\right) \equiv W$, $\operatorname{Lab}\left(u_{k}\right) \equiv U_{k}$, and $\operatorname{Lab}\left(p_{k}\right) \equiv A_{k}^{m_{k}}$.

Further one may assume that if $A_{k}$ is a period of some rank, then $\Delta_{k}$ has no cells compatible with $p_{k}$, since, by [41, Lemma 13.3], one may delete such a cell and

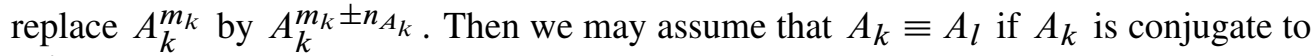
$A_{l}^{ \pm 1}$ in $G$. Since $|W|>\left|U_{k}\right|$, we also have $m_{k} \neq 0$.

Case 1 Assume that $m=\left|m_{k}\right| \leq 10 \zeta^{-1}$ for some $k$. Then $\left|A_{k}\right|=\left|A_{k}^{m}\right| / m \geq$ $0.1 \zeta|W| / 15=|W| / c>d_{r} / \phi(r)$. On the other hand, by Theorem 22.4 and Lemma 26.5 of [41], $\left|W U_{k}\right| \geq \bar{\beta} m\left|A_{k}\right|$, where $\bar{\beta}$ is the constant from Chapter 7 of [41] which is close to 1 . Hence $\left|A_{k}\right| \leq \bar{\beta}^{-1}(|W|+1)<2|W|=2|W|_{G}<\phi(r) d_{r}$.

Thus $\left|A_{k}\right| \in\left(d_{r} / \phi(r), d_{r} \phi(r)\right)$ and $\left|A_{k}\right|<2|W|=i$. It follows that $A_{k}$ cannot be simple in rank $i$. (Indeed otherwise it must be simple in all smaller ranks, and so it is conjugate in rank $j-1=\left|A_{k}\right|-1$ to a period (or to its inverse) of rank $j$ by the definition of the set $\mathcal{X}_{j}$.) Hence $A_{k}$ is a period of rank $j_{k}=\left|A_{k}\right|$, and $n_{A}=n_{0}$ because $\left|A_{k}\right| \in\left(d_{r} / \phi(r), d_{r} \phi(r)\right)$. By definition of $\mathcal{R}_{j_{k}}$, we have $A_{k}^{n_{0}}=1$ in $G$. Since $W U_{k}$ is conjugate to a power of $A_{k}$ in $G$, we also have $\left(W U_{k}\right)^{n_{0}}=1$, as desired. 
Case 2 We may now assume that $\left|m_{k}\right|>10 \xi^{-1}$ for $k=1, \ldots, 4$. Then for every pair $(k, l)(1 \leq k<l \leq 4)$, we identify diagram $\Delta_{k}$ with the mirror copy of $\Delta_{l}$ along the subpaths labeled by $W$ as we did this in the proof of Lemma 6.9. We obtain diagrams $\Gamma_{k l}$ with 3 boundary components $x_{k l}, v_{k l}^{1}, v_{k l}^{2}$ labeled by $X_{k l} \equiv U_{k} U_{l}^{-1}, A_{k}^{m_{k}}$, and $A_{l}^{-m_{l}}$, respectively. Denote by $\Delta_{k l}$ the reduced forms of diagrams $\Gamma_{k l}$.

Diagrams $\Delta_{k l}$ satisfy all conditions of $E$-maps defined in [41, Subsection 24.2] since $\left|v_{k l}^{1}\right|>10 \zeta^{-1}\left|A_{k}\right|,\left|v_{k l}^{2}\right|>10 \zeta^{-1}\left|A_{l}\right|$, and $\left|x_{k l}\right| \leq 2<\zeta \min \left(\left|v_{k l}^{1}\right|,\left|v_{k l}^{2}\right|\right)$. Lemma 24.6 of [41] says that for some $s, s^{\prime} \in\{1,2\}$, the $E$-map $\Delta_{k l}$ contains a contiguity submap $\Gamma^{k l}$ of $v_{k l}^{s}$ to $v_{k l}^{s^{\prime}}$ with contiguity degree $\left(v_{k l}^{s}, \Gamma^{k l}, v_{k l}^{s^{\prime}}\right)>0.1$, and also $\left(v_{k l}^{s^{\prime}}, \Gamma^{k l}, v_{k l}^{s}\right)>0.1$ if $s \neq s^{\prime}$

If $s=s^{\prime}$ then using Lemmas 21.1(1) and 25.8 from [41] for $\Gamma^{k l}$ we obtain a contradiction since $0.1\left|m_{t}\right|>\zeta^{-1}$ for $t=k, l$. Then we may assume that $s=1$ and $s^{\prime}=2$. Lemma 25.10 of [41] implies in turn that $A_{k} \equiv A_{l}$ and the cycles $v_{k l}^{1}$ and $v_{k l}^{2}$ are $A_{k}$-compatible, ie, the word $X_{k l}=\operatorname{Lab}\left(x_{k l}\right)$ is conjugate in $G$ to a power of $A_{k}$. These nontrivial in $F_{2}$ words $X_{k l}$ are nontrivial in $G$ by [41, Lemma 23.16].

Now we have $A_{1} \equiv \cdots \equiv A_{4} \equiv A$, and all $X_{k l}$ are conjugate to some powers $A^{m_{k l}}$ in $G$. By Lemma 26.5 and Theorem 22.4 of [41], $\left|m_{k l}\right||A|<\bar{\beta}^{-1}\left|X_{k l}\right| \leq 2 \bar{\beta}^{-1}<3$. It follows from this estimate and [41, Lemmas 22.1 and 23.16] that all $X_{k l}$ are conjugate to the powers of the same word $A$ in the free group $F_{2}$. But this is impossible because it is easy to see, that for four different words $U_{k}$ of length at most 1 , there are two words in the set $\left\{X_{k l}=U_{k} U_{l}^{-1}\right\}$ which are conjugate to $(a b)^{ \pm 1}$ and $\left(a b^{-1}\right)^{ \pm 1}$, respectively. Hence Case 2 is also impossible, and the lemma is proved.

Since every word in $\mathcal{X}_{i}$ has length $i$ and the set of defining relations of $G$ contains $\left\{A^{n_{0}} \mid A \in \mathcal{X}_{d_{r}}\right\}$, the following lemma gives Condition (c) of Proposition 6.2.

Lemma 6.11 The set $\mathcal{X}_{d_{r}}$ is nonempty for every large enough $r$. If $A \in \mathcal{X}_{d_{r}}$, then there exists no nonempty subword $w$ of the word $A^{n_{0}}$ such that $|w| \leq n_{0} d_{r} / 2$ and $w$ is equal in $G$ to a word of length $\leq|w| / 2$.

Proof The number of positive words of length $i$ in $\{a, b\}$ containing no nonempty subwords of the form $B^{6}$ is at least $(3 / 2)^{i}$ for $i \geq 1$ [41, Theorem 4.6]. Since the number of all words of length $i$ in $\{a, b\}$ is $2^{i}$ there is such 6 -aperiodic word $A$ of arbitrary length $i \gg 1$ which is not a proper power. (Indeed, for all large enough $i$, $(3 / 2)^{i}>2^{[i / 2]}+2^{[i / 3]}+2^{[i / 4]}+2^{[i / 5]}$.) Let $i=d_{r}$. It suffices to prove that $A$ is simple in rank $d_{r}-1$.

Arguing by contradiction, we have from the inductive definition that $A$ is conjugate in rank $i-1$ to a power of a period $B$ or some rank $j<i$ or to a power of a simple 
in rank $i-1$ word $B$ with $|B|<|A|$. In both cases we have a reduced diagram $\Delta$ of rank $i-1$ whose contours $p$ and $q$ are labeled by words $A$ and $B^{s}$, respectively. In the second case $\Delta$ has no cell compatible with $q$ [41, Section 13.3]. Hence $q$ is a smooth section of the B-map $\Delta$ by [41, Lemma 26.5].

If $\Delta$ has at least one cell, then there is a cell $\Pi$ in $\Delta$ with contiguity degree to $p$ greater than $1 / 2-\alpha-\gamma>\varepsilon$ [41, Lemma 21.7 and Corollary 22.2]). According to [41, Theorem 22.2] this implies that there is a contiguity subdiagram $\Gamma$ of rank 0 with $(\Pi, \Gamma, p) \geq \varepsilon$. This means than $A$ has a subword $B^{t}$, where $t=\left[\varepsilon n_{B} / 2\right]$. This contradicts the 6-aperiodicity of $A$ because $\varepsilon n_{B} \geq \varepsilon n_{0}>12$.

Thus $\Delta$ has no cells, that is the positive word $A$ is a power of some word of length $|B|<|A|$ in the free group $F_{2}$. This contradicts the choice of $A$, and the first statement of the lemma is proved.

Then assume that the word $w$ is equal to $v$ in $G$. If a reduced diagram $\Delta$ for this equality has rank $\geq d_{r}$, then its perimeter $|w|+|v|$ is at least $(1-\alpha) n_{0} d_{r}>\frac{3}{4} n_{0} d_{r}$ by [41, Lemma 23.16]. Since $|w| \leq n_{0} d_{r} / 2$ we obtain $|v| \geq|w| / 2$ as desired.

If $\operatorname{rank}(\Delta)<d_{r}$, then the section of the boundary $\partial \Delta$ labeled by $w$ is smooth by [41, Lemma 26.5]. It follows from [41, Theorem 22.4] that $|v|>(1-\beta)|w|>|w| / 2$, and the lemma is proved.

The group $G$ is infinite by [41, Theorem 26.1]. The order of arbitrary element of $G$ divides some $n_{A}$ by [41, Theorem 26.2], and so $G$ is a $p$-group according to our choice of the exponents $n_{A}$. The centralizers of nontrivial elements of $G$ are cyclic by [41, Theorem 26.5]. Thus we obtain Condition (d) of Proposition 6.2.

By Lemma 6.6, $\delta_{i_{r}} \leq n_{0} P\left(i_{r}\right)$. If $i_{r-1}<i<d_{r} / \phi(r)$, then, for a period $A$ of rank (and length) $i$, we have by definition of $n_{A}$, that $n_{A}|A| \leq p \max \left\{n_{0}, d_{r}\right\}$. If $d_{r} / \phi(r) \leq i \leq i_{r}=\left[d_{r} \phi(r)\right]$, then $n_{A}|A|=n_{0}|A| \leq n_{0} d_{r} \phi(r)$. Hence the obvious induction on $r$ shows that $P\left(i_{r}\right)=O\left(d_{r} \phi(r)\right)$. Thus $n_{0} P\left(i_{r}\right)=O\left(\phi(r) d_{r}\right)$, and property (e) of Proposition 6.2 is obtained too.

It is shown in [41, Theorem 26.2], that the order of every word $X$ does not exceed the order $n_{A}$ of a period $A$ of rank (and length) $i \leq|X|$. As above, we have that if $i_{r-1}<i<d_{r} / \phi(r)$, then $n_{A}=O\left(d_{r} / i_{r-1}\right)=O\left(d_{r} / d_{r-1}\right)=O\left(\phi(r)^{3}\right)$ by the definition of $d_{r}$ and Lemma 6.6. If $d_{r} / \phi(r) \leq i \leq i_{r}=\left[d_{r} \phi(r)\right]$, then $n_{A}=n_{0}=O(1)$. Since $i \geq i_{r-1} \geq r$, we have by induction on $r$ that $n(X)=n_{A}=o\left(\phi(|X|)^{3}\right)$, and property (f) is obtained. Since the Conditions (a), (b) and (c) are provided by Lemmas 6.7, 6.10 and 6.11, respectively, the proof of Proposition 6.2 is complete. 


\section{Open problems}

\subsection{Algebraic properties of lacunary hyperbolic groups}

Since the class of lacunary hyperbolic groups is very large, it would be interesting to establish more common properties of the groups in this class except those established in Section 3.1 and Section 3.4.

Here is a concrete problem.

Problem 7.1 Is it true that the growth of every nonelementary lacunary hyperbolic group is (a) exponential? (b) uniformly exponential?

Inspired by Theorem 3.18 and Corollary 3.21, it is natural to ask what kind of subgroups can lacunary hyperbolic groups have. In particular, we formulate the following:

Problem 7.2 Can a finitely generated non virtually cyclic subgroup of exponential growth of a lacunary hyperbolic group satisfy a nontrivial law?

Remark 7.3 The answer to Problem 7.2 is "no" for lacunary hyperbolic groups for which, using the notation of Remark 3.4, the injectivity radii $r_{i}$ are "much larger" than the hyperbolicity constants $\delta_{i}$. More precisely, let $G$ be a direct limit of groups $G_{i}$ and homomorphisms $\alpha_{i}: G_{i} \rightarrow G_{i+1}$ such that $G_{i}=\left\langle S_{i}\right\rangle, \alpha_{i}\left(S_{i}\right)=S_{i+1}, \Gamma\left(G_{i}, S_{i}\right)$ is $\delta_{i}$-hyperbolic, and the induced homomorphism $G_{i} \rightarrow G$ is injective on a ball of radius $r_{i}=\exp \exp \left(C \delta_{i}\right)$ for a large enough constant $C$. We claim that then a subgroup of exponential growth in $G$ cannot satisfy a nontrivial law.

Proof Indeed, let $H=\left\langle x_{1}, \ldots, x_{n}\right\rangle$ be a subgroup of $G$ having exponential growth. Let $b$ be the maximal length of an element $x_{i}$ in generators $S$ of $G$. Let $H_{i}=$ $\left\langle x_{1}(i), \ldots, x_{n}(i)\right\rangle$ be a preimage of $H$ in $G_{i}$. We can assume that $x_{j}(i)$ have length at most $b$ in $G_{i}$.

By [31, Propositions 3.2 and 5.5], for every $i \geq 1$, there exists a pair of elements $u_{i}, v_{i}$ of length at most $\exp \exp \left(C_{0} \delta_{i}\right)$ (for some uniform constant $\left.C_{0}\right)$ in the non virtually cyclic subgroup $H_{i}$, generating a free subgroup of $H_{i}$. Let $a_{i}, b_{i}$ be the images of $u_{i}, v_{i}$ in $G$. Let $l_{i}$ be the length of the shortest word in $\left\{a_{i}, b_{i}\right\}^{ \pm 1}$ that is equal to 1 in $G$. Then

$$
l_{i}>\frac{r_{i}}{\exp \exp \left(C_{0} \delta_{i}\right)} .
$$

Hence if we assume that $C>C_{0}$ we deduce that $\lim l_{i}=\infty$. Hence $H$ cannot satisfy any nontrivial law. 
Remark 7.4 It is easy to see that the lacunary hyperbolic groups from examples in Sections 3-5 can be chosen to satisfy the growth condition of Remark 7.3. Thus there are elementary amenable lacunary hyperbolic groups as well as groups with proper subgroups cyclic, torsion groups, groups with nontrivial centers, etc. satisfying this condition.

Problem 7.5 It is easy to construct a lacunary hyperbolic group with undecidable word problem (one can use a small cancellation nonrecursive presentation as in Proposition 3.12). But suppose that the word problem in a lacunary hyperbolic group $G$ is decidable. Does it imply that the conjugacy problem is decidable as well?

Using the known facts about solvability of the conjugacy problem in hyperbolic groups $[20 ; 2]$ it is easy to deduce that the answer is "yes" if the growth condition of Remark 7.3 holds.

It is also interesting to study linearity of lacunary hyperbolic groups. We do not know the answer to the following basic question.

Problem 7.6 Is every linear lacunary hyperbolic group hyperbolic?

\subsection{Asymptotic cones and finitely presented groups}

Theorem 4.17 and Theorem 5.11 proved in this paper provide us with a reach source of finitely generated groups all of whose asymptotic cones are locally isometric, but not all of them are isometric. Similar methods can be used to show that the groups from [51; 13, Section 7] also satisfy this property. However all these groups are infinitely presented. Moreover, in all our examples asymptotic cones are locally isometric to an $\mathbb{R}$-tree, which implies hyperbolicity for finitely presented groups by Proposition 3.2. However the following problem is still open.

Problem 7.7 Does there exist a finitely presented group all of whose asymptotic cones are locally isometric, but not all of them are isometric?

Note that finitely presented groups with different asymptotic cones were constructed in [44] (earlier, in [32], such groups were found under the assumption that the Continuum Hypothesis does not hold). 


\subsection{Asymptotic cones and amenability}

Another interesting problem is to find a characterization of groups all of whose asymptotic cones are locally isometric to an $\mathbb{R}$-tree in the spirit of Theorem 3.3. In particular, do such groups satisfy a suitable small (graded) cancellation condition? The affirmative answer to this question and the Kesten-Grigorchuk criterion for amenability would give an approach to the following.

Problem 7.8 Suppose that all asymptotic cones of a non-virtually cyclic group $G$ are locally isometric to an $\mathbb{R}$-tree. Does it follow that $G$ is nonamenable?

Below is another problem about asymptotic cones of amenable groups, which is still open.

Problem 7.9 Is there a finitely generated (resp. finitely presented) amenable nonvirtually cyclic group all (resp. some) of whose asymptotic cones have cut-points?

In particular, we do not know whether our groups from Section 3.5 have cut points in all asymptotic cones (for some choice of parameters).

\subsection{Divergence and Floyd boundary}

Problem 7.10 Is there a finitely presented group with divergence function $\operatorname{Div}(n, \delta)$ strictly between linear and quadratic for some $\delta$ ?

Recall that if the Floyd boundary $\partial G$ of a finitely generated group $G$ is nontrivial, $G$ acts on $\partial G$ as a convergence group [29]. On the other hand, geometrically finite convergence groups acting on nonempty perfect compact metric spaces are hyperbolic relative to the set of the maximal parabolic subgroups [52].

Problem 7.11 Suppose that a finitely generated group $G$ has a nontrivial Floyd boundary. Is $G$ hyperbolic relative to a collection of proper subgroups?

Note that if $G$ is hyperbolic relative to a collection of proper subgroups, then all asymptotic cones of $G$ are tree-graded with respect to some proper subsets. In particular, all asymptotic cones of $G$ have cut points. Thus Proposition 4.28 may be considered as an evidence towards the positive solution of Problem 7.11. 


\title{
7.5 Fundamental groups of asymptotic cones
}

The example of a group $G$ such that $\pi_{1}\left(\operatorname{Con}^{\omega}(G, d)\right)=\mathbb{Z}$ for some $d$ and $\omega$ allows us to realize any finitely generated free Abelian group as the fundamental group of $\operatorname{Con}^{\omega}(G, d)$ for a suitable $G$ by taking direct products of groups. On the other hand if $1 \rightarrow N \rightarrow G \rightarrow H \rightarrow 1$ is a finitely generated central extension and $N$ is endowed with the metric induced from $G$, then $\operatorname{Con}^{\omega}(N, d)$ has the structure of an Abelian topological group. Hence $\pi_{1}\left(\operatorname{Con}^{\omega}(N, d)\right)$ is Abelian. Thus there is no hope to construct asymptotic cones with countable non-Abelian groups by generalizing our methods. This leads to the following.

Problem 7.12 Does there exist a finitely generated group $G$ such that $\pi_{1}\left(\operatorname{Con}^{\omega}(G, d)\right)$ is countable (or, better, finitely generated) and non-Abelian for some (any) $d$ and $\omega$ ? Can $\pi_{1}\left(\operatorname{Con}^{\omega}(G, d)\right)$ be finite and nontrivial?

Note that for every countable group $C$ there exists a finitely generated group $G$ and an asymptotic cone $\operatorname{Con}^{\omega}(G, d)$ such that $\pi_{1}\left(\operatorname{Con}^{\omega}(G, d)\right)$ is isomorphic to the uncountable free power of $C$ [13, Theorem 7.33].

\section{Appendix: Finitely presented groups whose asymptotic cones are $\mathbb{R}$-trees}

\author{
Michael Kapovich AND BRUCE KLEINER ${ }^{1}$
}

The main result of this appendix is the following:

Theorem 8.1 Suppose that $G$ is a finitely presented group such that some asymptotic cone of $G$ is an $\mathbb{R}$-tree. Then $G$ is Gromov-hyperbolic.

This theorem will be an easy application of (a slightly modified version of) Gromov's local-to-global characterization of hyperbolic spaces.

Before proving Theorem 8.1, we will need several definitions and auxiliary results.

\footnotetext{
${ }^{1}$ The first author was supported in part by the NSF Grant DMS-04-05180 and the second author was supported in part by the NSF Grant DMS-07-01515.
} 


\section{Metric notions}

Given a metric space $Z$, let $B_{R}(z)$ denote the closed $R$-ball centered at $z$ in $Z$. A geodesic triangle $\Delta \subset Z$ is called $R$-thin if every side of $\Delta$ is contained in the $R$-neighborhood of the union of two other sides. A geodesic metric space $Z$ is called $\delta$-Rips-hyperbolic if each geodesic triangle in $Z$ is $\delta$-thin. (Rips was the first to introduce this definition.)

Let $Z$ be a metric space (not necessarily geodesic). For a basepoint $p \in Z$ define a number $\delta_{p} \in[0, \infty]$ as follows. For each $x \in Z$ set $|x|_{p}:=d(x, p)$ and

$$
(x, y)_{p}:=\frac{1}{2}\left(|x|_{p}+|y|_{p}-d(x, y)\right) .
$$

Then

$$
\delta_{p}:=\inf _{\delta \in[0, \infty]}\left\{\delta \mid \forall x, y, z \in Z,(x, y)_{p} \geq \min \left((x, z)_{p},(y, z)_{p}\right)-\delta\right\} .
$$

We say that $Z$ is $\delta$-Gromov-hyperbolic, if $\infty>\delta \geq \delta_{p}$ for some $p \in X$. We note that if $Z$ a geodesic metric space which is $\delta$-Gromov-hyperbolic then $Z$ is $4 \delta$-Ripshyperbolic and vice-versa (see Gromov [20, 6.3C]).

A metric space $Z$ is said to have bounded geometry if there exists a function $\phi(r)$ such that every $r$-ball in $Z$ contains at most $\phi(r)$ points. For instance, every finitely generated group $G$ with a word-metric has bounded geometry.

\section{Rips complexes}

Given a metric space $Z$, let $P_{d}(Z)$ denote the $d$-Rips complex, ie, the complex whose $k$-simplices are $k+1$-tuples of points in $Z$, which are within distance $\leq d$ from each other. We equip the Rips complex $P_{d}(Z)$ with a path metric for which each simplex is path-isometric to a regular Euclidean simplex of side length $d$.

Given a cell complex $X$, we let $X^{i}$ denote the $i$-skeleton of $X$.

Lemma 8.2 Let $G=\langle A \mid \mathcal{R}\rangle$ be a finitely presented group, $D$ the length of the longest relation in $\mathcal{R}$. Then $P_{d}(G)$ is simply connected for all $d \geq D$.

Proof Let $Y$ be the Cayley complex of this presentation, ie the universal cover of the presentation complex of $\langle A \mid R\rangle$. Then $Y^{0}=G$ and $Y^{1}$ is the Cayley graph of $G$ (with respect to the generating set $A$ ).

First of all, $P_{d}(G)$ is connected for each $d \geq 1$. We note that $Y^{1}=P_{1}(G)$. Since $\pi_{1}\left(Y^{1}\right)$ is generated by the boundaries of the 2 -cells in $Y$, it is clear that the map

$$
\pi_{1}\left(Y^{1}\right) \rightarrow \pi_{1}\left(P_{d}(G)\right)
$$

is trivial for $d \geq D$. Vanishing of $\pi_{1}\left(P_{d}(G)\right)$ however is slightly less obvious. 
Let $d \geq 1$. Consider a loop $\gamma: S^{1} \rightarrow P_{d}^{1}(G)$. After homotoping $\gamma$ if necessary, we may assume that it is a simplicial map with respect to some triangulation $\mathcal{T}$ of $S^{1}$. Define a map $\gamma_{1}: S^{1} \rightarrow Y^{1}$ as follows. For each vertex $v$ of $\mathcal{T}$, let $\gamma_{1}(v) \in G=P_{d}^{0}(G)$ be equal to $\gamma(v)$. For each edge $e=\left[v_{1} v_{2}\right]$ of $\mathcal{T}$, let $\left.\gamma_{1}\right|_{e}$ be a geodesic in $Y^{1}$ between $\gamma_{1}\left(v_{1}\right)$ and $\gamma_{1}\left(v_{2}\right)$. There is a natural map

$$
Y^{1} \stackrel{i_{1}}{\rightarrow} P_{d}^{1}(G)
$$

which takes each $v \in G=Y^{0}$ to the corresponding vertex of $P_{d}^{0}(G)$ and maps each edge of $Y^{1}$ at constant speed to the corresponding edge of $P_{d}^{1}(G)$. Let $\gamma_{2}:=i_{1} \circ \gamma_{1}$. If $d \geq D$ then $i_{1}$ can be extended to a map

$$
Y \stackrel{i_{2}}{\rightarrow} P_{d}^{2}(G)
$$

Since $Y$ is simply connected, this implies that $\gamma_{2}$ is null-homotopic in $P_{d}^{2}(G)$.

On the other hand, we claim that $\gamma_{2}$ is homotopic to $\gamma$ in $P_{d}^{2}(G)$. To see this, for each edge $e=\left[v_{1} v_{2}\right]$ of $\mathcal{T}$, let $y_{0}=\gamma(v), y_{1}, \ldots, y_{m}=\gamma(w)$ be the vertices of $Y^{1}$ on $\gamma_{1}(e)$ so that $\gamma_{2}(e)$ is the concatenation of the edges

$$
\left[y_{0} y_{1}\right], \ldots,\left[y_{m-1} y_{m}\right] \subset P_{d}^{2}(G) .
$$

Since $\gamma_{1}(e)$ is a geodesic between $y_{0}, y_{m}$ and $d_{Y^{1}}\left(y_{0}, y_{m}\right) \leq d$, we get:

$$
d_{Y^{1}}\left(y_{0}, y_{i}\right) \leq d, \quad i=1, \ldots, m-1 .
$$

Hence each triple of vertices $y_{0}, y_{i}, y_{m}$ spans a 2-simplex $\Delta_{i}$ in $P_{d}^{2}(G)$. Together these simplices define a homotopy between $\gamma(e)$ and $\gamma_{2}(e)$ (rel. the endpoints). Thus the loops $\gamma$ and $\gamma_{2}$ are homotopic.

\section{Coarse Cartan-Hadamard theorem}

Our main technical result is the following coarse Cartan-Hadamard theorem for Gromovhyperbolic spaces:

Theorem 8.3 (cf [20], [6, Theorem 8.1.2]) There are constants $C_{1}, C_{2}$, and $C_{3}$ with the following property. Let $Z$ be a metric space of bounded geometry. Assume that for some $\delta$, and $d \geq C_{1} \delta$, every ball of radius $C_{2} d$ in $Z$ is $\delta$-Gromov-hyperbolic, and $P_{d}(Z)$ is 1 -connected. Then $Z$ is $C_{3} d$-Gromov-hyperbolic.

One can give a direct proof of this theorem modeled on the proof of the CartanHadamard theorem. Instead of doing this, we will use 6.8.M and 6.8.N from [20]. In 
brief, the idea of the proof is to translate Gromov's local-to-global result in [20], which is expressed using isoperimetric information, into one using $\delta$-hyperbolicity.

Consider the $d$-Rips complex $P_{d}(Z)$ of $Z$. Given a polygonal loop $c$

$$
c: S^{1} \rightarrow P_{d}^{1}(Z),
$$

let $L(c)$ denote the length of $c$ and let $A(c)$ be the least area of a simplicial disk

$$
f: D^{2} \rightarrow P_{d}^{2}(Z)
$$

so that $f \mid \partial D^{2}=c$. If such disk does not exist, we set $A(c)=\infty$. Note that in order to retain the proper scaling behavior, the length and area are computed here using the metric on $P_{d}^{1}(Z)$ and $P_{d}^{2}(Z)$ rather than the combinatorial length and area.

Taking $A_{0}^{\prime}=500 d^{2}$ in $[20,6.8 . \mathrm{M}]$ we get:

Proposition 8.4 ([20, 6.8.M], adapted version) Suppose that $Z$ is a metric space of bounded geometry, such that for some $d \geq 0$ every simplicial circle $S^{\prime}$ in $P_{d}^{1}(Z)$ with

$$
500 d^{2} \leq A\left(S^{\prime}\right) \leq 64\left(500 d^{2}\right)
$$

satisfies

$$
L\left(S^{\prime}\right) \geq d \sqrt{(4000)(64)(500)}
$$

and $P_{d}(Z)$ is 1 -connected. Then $P_{d}^{1}(Z)$ is (400) $\sqrt{500} d$-Rips-hyperbolic [20, 6.8.J] and $Z$ is (400) $\sqrt{500} d$-Gromov-hyperbolic.

Theorem 6.8.N from [20] states:

Proposition 8.5 [20, Theorem 6.8.N] If $Z$ is $\delta$-Gromov-hyperbolic and $d \geq 8 \delta$, then every simplicial circle $S^{\prime} \subset P_{d}^{1}(Z)$ satisfies $L\left(S^{\prime}\right) \geq(d /(4 \sqrt{3})) A\left(S^{\prime}\right)$.

Proof of Theorem 8.3 Since the statement of the theorem is scale invariant, after rescaling the metric we may assume that

$$
\frac{500 d^{2}}{4 \sqrt{3}} \geq \sqrt{(4000)(64)(500)} .
$$

Let $C_{1}:=32$ and $C_{2}:=64 \cdot 500$. Let $S^{\prime} \subset P_{d}^{1}(Z)$ be a simplicial circle with

$$
500 d^{2} \leq A\left(S^{\prime}\right) \leq 64\left(500 d^{2}\right)
$$


and let $f: D \rightarrow P_{d}^{2}(Z)$ be a least area simplicial 2-disk filling $S^{\prime}$. By (36), there are at most (64)(500) triangles in the triangulated 2-disk $D$ which are mapped isomorphically by $f$. Therefore, if we look at $\operatorname{Im}(f) \subset P_{d}^{2}(Z)$, and let $W \subset \operatorname{Im}(f)$ be the closure of the union of 2-simplices contained in $\operatorname{Im}(f)$, then connected components $W_{i}$ of $W$ have diameter $\leq(64)(500) d$. This means that we can decompose $D$ along disjoint arcs as the union of disks $D_{i}, 1 \leq i \leq k+1$ and regions $E_{j}$, so that each $f\left(E_{j}\right)$ is at most 1-dimensional and the diameter of each "minimal 2-disk" $f\left(D_{i}\right)$ is at most (64)(500)d. This decomposition corresponds to the van Kampen diagram associated with $f$.

By assumption, every ball of radius $C_{2} d=64 \cdot 500 d$ is $\delta$-hyperbolic and

$$
d \geq C_{1} \delta=32 \delta,
$$

so by applying Proposition 8.5 to $f\left(\partial D_{1}\right), \ldots, f\left(\partial D_{k+1}\right)$ and adding up the results, we obtain

$$
L\left(S^{\prime}\right) \geq \frac{d}{4 \sqrt{3}} A\left(S^{\prime}\right) \geq \frac{d}{4 \sqrt{3}} 500 d^{2} \geq d \sqrt{(4000)(64)(500)}
$$

where the last inequality comes from (35). By Proposition 8.4 we conclude that $Z$ is $C_{3} d$-Gromov-hyperbolic where $C_{3}:=(400)(\sqrt{500})$.

Corollary 8.6 There exist a constant $0<c<\infty$ such that for each finitely presented group $G$ there exists a constant $\rho$ (depending on the presentation) with the property:

Suppose that for some $R \geq \rho$, each ball $B_{R}(y) \subset Y^{1}$ is $c R$-hyperbolic, where $Y^{1}$ is the Cayley graph of $G$. Then $G$ is Gromov-hyperbolic.

Proof The complex $P_{d}^{2}(G)$ is simply connected for each $d \geq D$ (see Lemma 8.2), where $D$ is the length of the longest relator in the presentation of $G$. Let $C_{1}, C_{2}$ be the constants from Theorem 8.3, where $Z=G$ with the word metric. Choose $\rho$ so that $\rho / C_{2}=D$. Let $c:=1 /\left(4 C_{1} C_{2}\right)$.

For $R \geq \rho$ set $d:=R / C_{2}$ and $\delta:=c R$. Since

$$
d=\frac{R}{C_{2}} \geq \frac{\rho}{C_{2}}=D,
$$

the complex $P_{d}^{2}(G)$ is 1 -connected; see Lemma 8.2. We now verify the assumptions of Theorem 8.3.

First, by our choice of the constant $c$,

$$
d=\frac{R}{C_{2}} \geq 4 C_{1} c R=C_{1}(4 \delta) .
$$

In fact, the equality holds. 
Next, by the assumption of Corollary 8.6, each ball $B_{R}(y) \subset Y^{1}$ is $c R=\delta$-Ripshyperbolic. Therefore for each $x \in G$, the ball $B_{R}(x) \subset G$ is $4 \delta$-Gromov-hyperbolic. Since $C_{2} d=R$, every $C_{2} d$-ball in $G$ is $4 \delta$-Gromov-hyperbolic.

Theorem 8.3 now implies that $Y^{1}$ is Gromov-hyperbolic.

\section{Proof of Theorem 8.1}

Let $\omega$ be a nonprincipal ultrafilter on $\mathbb{N}$, let $R_{j}$ be a sequence of positive real numbers such that $\lim ^{\omega} R_{j}=\infty$. Let $Y$ be the Cayley complex of a finite presentation of $G$.

Let $y_{j} \in G$ be a sequence. By our assumption, the asymptotic cone $\lim ^{\omega}\left(1 / R_{j}\right)\left(Y^{1}, y_{j}\right)$ is a tree for some choice of $\omega$ and $\left(R_{j}\right)$. Thus each geodesic triangle in an $R_{j}$-ball $B\left(y_{j}, R_{j}\right) \subset Y^{1}$ is $\delta_{j}$-thin, where $\lim ^{\omega}\left(\delta_{j} / R_{j}\right)=0$. Hence the same is true for each ball $B\left(y, R_{j}\right) \subset Y^{1}, y \in G$. For sufficiently large $j, R_{j} \geq \rho=\rho(Y)$ and $\delta_{j} / R_{j}<c$, where $\rho, c$ are the constants from the previous corollary. Hence, by Corollary 8.6, the graph $Y^{1}$ is Gromov-hyperbolic and therefore $G$ is too.

\section{References}

[1] S I Adian, The Burnside problem and identities in groups, Ergebnisse der Math. und ihrer Grenzgebiete 95, Springer, Berlin (1979) MR537580 Translated from the Russian by $\mathrm{J}$ Lennox and $\mathbf{J}$ Wiegold

[2] J M Alonso, T Brady, D Cooper, V Ferlini, M Lustig, M Mihalik, H Short (editor), Notes on word hyperbolic groups, from: "Group theory from a geometrical viewpoint (Trieste, 1990)", (E Ghys, A Haefliger, A Verjovsky, editors), World Sci. Publ., River Edge, NJ (1991) 3-63 MR1170363

[3] G Arzhantseva, A Minasyan, D V Osin, The SQ-universality and residual properties of relatively hyperbolic groups, J. Algebra 315 (2007) 165-177 MR2344339

[4] W Ballmann, Lectures on spaces of nonpositive curvature, DMV Seminar 25, Birkhäuser Verlag, Basel (1995) MR1377265 With an appendix by M Brin

[5] J A Behrstock, Asymptotic geometry of the mapping class group and Teichmüller space, Geom. Topol. 10 (2006) 1523-1578 MR2255505

[6] B H Bowditch, Notes on Gromov's hyperbolicity criterion for path-metric spaces, from: "Group theory from a geometrical viewpoint (Trieste, 1990)", (E Ghys, A Haefliger, A Verjovsky, editors), World Sci. Publ., River Edge, NJ (1991) 64-167 MR1170364

[7] M R Bridson, A Haefliger, Metric spaces of non-positive curvature, Grund. der Math. Wissenschaften 319, Springer, Berlin (1999) MR1744486

[8] J Burillo, Dimension and fundamental groups of asymptotic cones, J. London Math. Soc. (2) 59 (1999) 557-572 MR1709665 
[9] L van den Dries, A J Wilkie, Gromov's theorem on groups of polynomial growth and elementary logic, J. Algebra 89 (1984) 349-374 MR751150

[10] C Druţu, Relatively hyperbolic groups: geometry and quasi-isometric invariance, to appear in Comm. Math. Helv. arXiv:math/0605211

[11] C Druţu, Quasi-isometry invariants and asymptotic cones, from: "International Conference on Geometric and Combinatorial Methods in Group Theory and Semigroup Theory (Lincoln, NE, 2000)", Internat. J. Algebra Comput. 12 (2002) 99-135 MR1902363

[12] C Druţu, S Mozes, M V Sapir, Divergence in lattices in semisimple Lie groups and graphs of groups, to appear in Trans. Amer. Math. Soc. arXiv:0801.4141

[13] C Druţu, M V Sapir, Tree-graded spaces and asymptotic cones of groups, Topology 44 (2005) 959-1058 MR2153979 With an appendix by D V Osin and Sapir

[14] C Druţu, M V Sapir, Groups acting on tree-graded spaces and splittings of relatively hyperbolic groups, Adv. Math. 217 (2008) 1313-1367 MR2383901

[15] A Erschler, D V Osin, Fundamental groups of asymptotic cones, Topology 44 (2005) 827-843 MR2136537

[16] W J Floyd, Group completions and limit sets of Kleinian groups, Invent. Math. 57 (1980) 205-218 MR568933

[17] S M Gersten, Bounded cocycles and combings of groups, Internat. J. Algebra Comput. 2 (1992) 307-326 MR1189238

[18] É Ghys, P de la Harpe, Espaces métriques hyperboliques, from: "Sur les groupes hyperboliques d'après Mikhael Gromov (Bern, 1988)”, Progr. Math. 83, Birkhäuser Boston, Boston, MA (1990) 27-45 MR1086650

[19] M Gromov, Groups of polynomial growth and expanding maps, Inst. Hautes Études Sci. Publ. Math. (1981) 53-73 MR623534

[20] M Gromov, Hyperbolic groups, from: "Essays in group theory", (S Gersten, editor), Math. Sci. Res. Inst. Publ. 8, Springer, New York (1987) 75-263 MR919829

[21] M Gromov, Asymptotic invariants of infinite groups, from: "Geometric group theory, Vol. 2 (Sussex, 1991)”, (G A Niblo, M A Roller, editors), London Math. Soc. Lecture Note Ser. 182, Cambridge Univ. Press (1993) 1-295 MR1253544

[22] M Gromov, Random walk in random groups, Geom. Funct. Anal. 13 (2003) 73-146 MR1978492

[23] P Hall, The Edmonton notes on nilpotent groups, Queen Mary College Math. Notes, Math. Dept., Queen Mary College, London (1969) MR0283083

[24] G Higman, Amalgams of p-groups, J. Algebra 1 (1964) 301-305 MR0167527

[25] S V Ivanov, The free Burnside groups of sufficiently large exponents, Internat. J. Algebra Comput. 4 (1994) ii+308 MR1283947 
[26] S V Ivanov, A Y Ol'shanskii, Hyperbolic groups and their quotients of bounded exponents, Trans. Amer. Math. Soc. 348 (1996) 2091-2138 MR1327257

[27] M Kapovich, B Kleiner, B Leeb, Quasi-isometries and the de Rham decomposition, Topology 37 (1998) 1193-1211 MR1632904

[28] M Kapovich, B Leeb, 3-manifold groups and nonpositive curvature, Geom. Funct. Anal. 8 (1998) 841-852 MR1650098

[29] A Karlsson, Free subgroups of groups with nontrivial Floyd boundary, Comm. Algebra 31 (2003) 5361-5376 MR2005231

[30] B Kleiner, B Leeb, Rigidity of quasi-isometries for symmetric spaces and Euclidean buildings, Inst. Hautes Études Sci. Publ. Math. (1997) 115-197 (1998) MR1608566

[31] M Koubi, Croissance uniforme dans les groupes hyperboliques, Ann. Inst. Fourier (Grenoble) 48 (1998) 1441-1453 MR1662255

[32] L Kramer, S Shelah, K Tent, S Thomas, Asymptotic cones of finitely presented groups, Adv. Math. 193 (2005) 142-173 MR2132762

[33] R C Lyndon, P E Schupp, Combinatorial group theory, Ergebnisse der Math. und ihrer Grenzgebiete 89, Springer, Berlin (1977) MR0577064

[34] A I Mal'cev, Model correspondences, Izv. Akad. Nauk SSSR. Ser. Mat. 23 (1959) 313-336 MR0120152

[35] K V Mikhajlovskii, A Y Ol'shanskii, Some constructions relating to hyperbolic groups, from: "Geometry and cohomology in group theory (Durham, 1994)", (P H Kropholler, G A Niblo, R Stöhr, editors), London Math. Soc. Lecture Note Ser. 252, Cambridge Univ. Press (1998) 263-290 MR1709962

[36] I Mineyev, Straightening and bounded cohomology of hyperbolic groups, Geom. Funct. Anal. 11 (2001) 807-839 MR1866802

[37] W D Neumann, L Reeves, Central extensions of word hyperbolic groups, Ann. of Math. (2) 145 (1997) 183-192 MR1432040

[38] Y Ollivier, Growth exponent of generic groups, Comment. Math. Helv. 81 (2006) 569-593 MR2250854

[39] A Y Ol'shanskii, An infinite simple torsion-free Noetherian group, Izv. Akad. Nauk SSSR Ser. Mat. 43 (1979) 1328-1393 MR567039

[40] A Y Ol'shanskii, An infinite group with subgroups of prime orders, Izv. Akad. Nauk SSSR Ser. Mat. 44 (1980) 309-321, 479 MR571100

[41] A Y Ol'shanskii, Geometry of defining relations in groups, Math. and its Applications (Soviet Ser.) 70, Kluwer, Dordrecht (1991) MR1191619 Translated from the 1989 Russian original by Yu A Bakhturin

[42] A Y Ol'shanskii, On residualing homomorphisms and G-subgroups of hyperbolic groups, Internat. J. Algebra Comput. 3 (1993) 365-409 MR1250244 
[43] A Y Ol'shanskii, M V Sapir, Groups with small Dehn functions and bipartite chord diagrams, Geom. Funct. Anal. 16 (2006) 1324-1376 MR2276542

[44] A Y Ol'shanskii, M V Sapir, A finitely presented group with two non-homeomorphic asymptotic cones, Internat. J. Algebra Comput. 17 (2007) 421-426 MR2310154

[45] D V Osin, Kazhdan constants of hyperbolic groups, Funktsional. Anal. i Prilozhen. 36 (2002) 46-54 MR1958994

[46] D V Osin, Relatively hyperbolic groups: intrinsic geometry, algebraic properties, and algorithmic problems, Mem. Amer. Math. Soc. 179 (2006) vi+100 MR2182268

[47] P Papasoglu, On the asymptotic cone of groups satisfying a quadratic isoperimetric inequality, J. Differential Geom. 44 (1996) 789-806 MR1438192

[48] F Point, Groups of polynomial growth and their associated metric spaces, J. Algebra 175 (1995) 105-121 MR1338969

[49] J Rosenberg, $C^{*}$-algebras, positive scalar curvature, and the Novikov conjecture, Inst. Hautes Études Sci. Publ. Math. (1983) 197-212 (1984) MR720934

[50] G Skandalis, J L Tu, G Yu, The coarse Baum-Connes conjecture and groupoids, Topology 41 (2002) 807-834 MR1905840

[51] S Thomas, B Velickovic, Asymptotic cones of finitely generated groups, Bull. London Math. Soc. 32 (2000) 203-208 MR1734187

[52] A Yaman, A topological characterisation of relatively hyperbolic groups, J. Reine Angew. Math. 566 (2004) 41-89 MR2039323

AYuO, DVO, MVS: Department of Mathematics, Vanderbilt University

Nashville, TN 37240, USA

AYuO: Department of Mathematics, Moscow State University

Moscow 119899, Russia

Michael Kapovich

MK: Department of Mathematics, University of California

Davis, CA 95616, USA

\section{Bruce Kleiner}

BK: Department of Mathematics, Courant Institute, New York University 251 Mercer Street, New York, NY 10012, USA

alexander.olshanskiy@vanderbilt.edu, denis.v.osin@vanderbilt.edu, m.sapir@vanderbilt.edu, kapovich@math.ucdavis.edu, bkleiner@cims.nyu.edu

Proposed: Benson Farb Received: 17 July 2007 Seconded: Dmitri Burago, Walter Neumann 